\title{
CURRENTS AND THEIR COUPLINGS IN THE WEAK SECTOR OF THE STANDARD MODEL
}

\author{
I. S. Towner and J. C. Hardy \\ AECL, Chalk River Laboratories, Chalk River \\ Ontario KOJ 1J0, Canada
}

\begin{abstract}
Beta-decay and muon-capture experiments in nuclei are reviewed. The conserved vector current hypothesis is confirmed through the observed constancy of the vector coupling constant determined from the superallowed Fermi transitions and from the measurement of the weak-magnetism term in mirror Gamow-Teller transitions. The axial-vector and pseudoscalar coupling constants in the nucleon are determined from neutron decay and muon capture on the proton respectively. In finite nuclei, evidence for these coupling constants being reduced relative to their free-nucleon values is discussed. Meson-exchange currents are shown to be an important correction to the time-like part of the axial current as evident in first-forbidden beta decays. Tests of the Standard Model are discussed, as well as extensions beyond it involving right-hand currents and scalar interactions.
\end{abstract}

\section{Introduction}

\subsection{Standard Model for Quarks and Leptons}

The standard electroweak model is based on the gauge groupl $S U(2) \times U(1)$, with four vector gauge bosons $W_{\mu}^{a}, a=1,2,3$, and $B_{\mu}$ for the $S U(2)$ and $U(1)$ sectors respectively, and the corresponding gauge coupling constants $g$ and $g^{\prime}$. The left-handed fermion fields, $\psi_{i}=\left(\nu_{i}, \ell_{i}^{-}\right)$and $\left(u_{i}, d_{i}^{\prime}\right)$, comprise neutrinos, $\nu_{i}$, leptons, $\ell_{i}^{-}$, up and down quarks, $u_{i}$ and $d_{i}^{\prime}$ for the $i^{\text {th }}$ fermion family, and transform as doublets under $S U(2)$, where $d_{i}^{\prime} \equiv \sum_{j} V_{i j} d_{j}$ and $V$ is the Cabibbo-Kobayashi-Maskawa (CKM) matrix, which we discuss shortly. The right-handed fields are $S U(2)$ singlets. The interaction part of the Lagrangian for quarks and leptons is \#1

$$
\begin{aligned}
\mathcal{L}_{\text {int }} & =\frac{i g}{2 \sqrt{2}} \sum_{i} \bar{\psi}_{i} \gamma_{\mu}\left(1+\gamma_{5}\right)\left(T^{+} W_{\mu}^{+}+T^{-} W_{\mu}^{-}\right) \psi_{i} \\
& +i e \sum_{i} \bar{\psi}_{i} q_{i} \gamma_{\mu} \psi_{i} A_{\mu} \\
& +\frac{i e}{2 \cos \theta_{\mathrm{w}}} \sum_{i} \bar{\psi}_{i} \gamma_{\mu}\left(V^{i}+A^{i} \gamma_{5}\right) \psi_{i} Z_{\mu}
\end{aligned}
$$

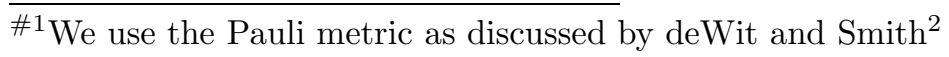


where $\theta_{\mathrm{w}} \equiv \tan ^{-1}\left(g^{\prime} / g\right)$ is the weak angle, $e=g \sin \theta_{\mathrm{w}}$ is the positron electric charge (and hence is positive) and $A_{\mu} \equiv B_{\mu} \cos \theta_{\mathrm{W}}+W_{\mu}^{3} \sin \theta_{\mathrm{W}}$ is the massless photon field. Further, $W^{ \pm} \equiv\left(W^{1} \mp i W^{2}\right) / 2$ and $Z=-B \sin \theta_{\mathrm{w}}+W^{3} \cos \theta_{\mathrm{w}}$ are the massive charged and neutral weak boson fields, respectively, while $T^{+}$and $T^{-}$are the weak isospin raising and lowering operators. The vector and axial couplings are

$$
\begin{aligned}
V^{i} & \equiv t_{3 L}(i)-2 q_{i} \sin ^{2} \theta_{\mathrm{w}} \\
A^{i} & \equiv t_{3 L}(i)
\end{aligned}
$$

where $t_{3 L}(i)$ is the weak isospin of fermion $i\left(+\frac{1}{2}\right.$ for $u_{i}$ and $\nu_{i} ;-\frac{1}{2}$ for $d_{i}$ and $\ell_{i}$ ) and $q_{i}$ is the charge of $\psi_{i}$ in units of $e$.

The three terms in the interaction Lagrangian of Eq.11 represent the chargedcurrent weak interaction, the electromagnetic interaction and the neutral-current weak interaction respectively. Note that the Lorentz structure involves only vectors and axial vectors; there is no compelling experimental evidence for scalars, pseudoscalars or tensor constructions. The minimal Standard Model as described by Eq. 1 successfully explains $W$ and $Z$ decays, neutrino-hadron scattering, neutrino-electron scattering and parity-violating electron-hadron neutral-current experiments, providing that radiative corrections to order $\alpha$, the fine-structure constant, are applied. In this chapter we will focus our discussion on the charged-current weak interaction in semi-leptonic decays involving quarks and leptons from the first family. As an example, consider the decay $d \rightarrow u e^{-} \bar{\nu}_{e}$, for which the $T$-matrix is 2

$$
T_{f i}=\frac{g^{2}}{8} V_{u d} \bar{\psi}_{u} \gamma_{\mu}\left(1+\gamma_{5}\right) \psi_{d} \frac{\delta_{\mu \nu}+k_{\mu} k_{\nu} / m_{\mathrm{w}}^{2}}{k^{2}+m_{\mathrm{w}}^{2}} \bar{\psi}_{e^{-}} \gamma_{\nu}\left(1+\gamma_{5}\right) \psi_{\bar{\nu}_{e}}
$$

where $k$ is the momentum transferred between the hadrons and leptons. For momenta small compared to the $W$-boson mass, $m_{\mathrm{W}}$, which is typically the case for leptons and quarks of the first family, $T_{f i}$ reduces to

$$
\begin{aligned}
T_{f i} & =\frac{G_{\mathrm{F}}}{\sqrt{2}} V_{u d} \bar{\psi}_{u} \gamma_{\mu}\left(1+\gamma_{5}\right) \psi_{d} \bar{\psi}_{e^{-}} \gamma_{\mu}\left(1+\gamma_{5}\right) \psi_{\bar{\nu}_{e}} \\
& \equiv-\frac{G_{\mathrm{F}}}{\sqrt{2}} V_{u d} J_{\mu}^{h} J_{\mu}^{\ell},
\end{aligned}
$$

which is the familiar effective four-fermion interaction with the Fermi constant given by $G_{\mathrm{F}} / \sqrt{2}=g^{2} /\left(8 m_{\mathrm{w}}^{2}\right)$. Here $J_{\mu}^{h}$ and $J_{\mu}^{\ell}$ are known as the hadron and lepton currents, where

$$
J_{\mu}^{h}=i \bar{\psi}_{u} \gamma_{\mu}\left(1+\gamma_{5}\right) \psi_{d} \equiv V_{\mu}^{h}+A_{\mu}^{h}
$$

and analogously for $J_{\mu}^{\ell}$. 
Table 1. Parameters of the CKM matrix

\begin{tabular}{ll}
\hline Value & Source \\
\hline$\left|V_{u d}\right|=0.9741 \pm 0.0017$ & Nuclear beta decay relative to muon decay \\
$\left|V_{u s}\right|=0.2205 \pm 0.0018$ & $K_{e 3}$ and hyperon decays \\
$\left|V_{c d}\right|=0.204 \pm 0.017$ & Neutrino production of charm from valence $d$ quarks \\
$\left|V_{c s}\right|=1.01 \pm 0.18$ & $D \rightarrow \bar{K} e^{+} \nu_{e}$ and $D$ lifetimes \\
$\left|V_{u b} / V_{c b}\right|=0.08 \pm 0.02$ & Energy spectrum for semileptonic decay of $B$ mesons \\
$\left|V_{c b}\right|=0.040 \pm 0.005$ & $\bar{B} \rightarrow D^{*} \ell \bar{\nu}_{\ell}$ and $B$ lifetimes \\
\hline
\end{tabular}

\subsection{Cabibbo-Kobayashi-Maskawa Matrix}

The up-quark, $u$, obtained in Eq. 3 by the explicit operation of the weak interaction, $\gamma_{\mu}\left(1+\gamma_{5}\right)$, upon the down-quark, $d$, may not be the physically observed up-quark. That is, the quark mass eigenstates are not the same as the weak interaction eigenstates \#2. The matrix relating the two bases was defined for three families and given an explicit parameterization by Kobayashi and Maskawall. It generalizes the two-family case where the matrix is parameterized by a single angle: the Cabibbo angle

By convention, the three quarks with charge $2 / 3(u, c$ and $t)$ are unmixed and all the mixing is expressed in terms of a $3 \times 3$ unitary matrix $V$ operating on the quarks with charge $-1 / 3(d, s, b)$ :

$$
\left(\begin{array}{c}
d^{\prime} \\
s^{\prime} \\
b^{\prime}
\end{array}\right)=\left(\begin{array}{ccc}
V_{u d} & V_{u s} & V_{u b} \\
V_{c d} & V_{c s} & V_{c b} \\
V_{t d} & V_{t s} & V_{t b}
\end{array}\right)\left(\begin{array}{c}
d \\
s \\
b
\end{array}\right) .
$$

The values of individual matrix elements can in principle be determined from weak decays of the relevant quarks. The Particle Data Group's recommended values listed in Table 1 except for $V_{u d}$, which we will have more to say about later in this chapter. By construction, the CKM matrix is unitary so its nine elements are not independent of each other. Typical parameterizations of the matrix involve three angles and one phase. In the Standard Model, the explanation of the observed CP violation requires such a phase and hence requires at least three families of quarks.

The unitarity of the CKM matrix is most stringently tested experimentally in the first row of the matrix:

$$
\left|V_{u d}\right|^{2}+\left|V_{u s}\right|^{2}+\left|V_{u b}\right|^{2}=1 .
$$

\footnotetext{
\#2 In the limit that quark masses are zero, the two bases will be identical and no CKM matrix is required. In the lepton sector we will not consider any ramifications of the possibility that neutrino masses are non-zero and hence not introduce any CKM matrix in the lepton sector.
} 
This test is dominated by the accuracy with which $V_{u d}$ can be determined. A failure of the test could signal an extension to the Standard Model, such as extra $U(1)$ groups or right-handed, $\gamma_{\mu}\left(1-\gamma_{5}\right)$, weak-interaction couplings. We return to these points later.

\subsection{Lagrangian for Nucleons}

In nuclear physics, a similar Lagrangian for nucleons is required with the nucleons treated as elementary spin-1/2 fermions. The Standard Model interaction Lagrangian of Eq.1 may not be immediately applicable for this case, however, since this elementary Lagrangian is explicitly constructed for, and tested in, interactions of pointlike fermions. Nucleons, by contrast, are composite particles of three quarks. To treat them as elementary spin-1/2 fermions requires some modification to the elementary Lagrangian as can be seen from the following argument.

Consider the time component of the vector part of the hadron current (see Eq. .5), $V_{0}^{h}=\bar{\psi}_{u} \gamma_{4} \psi_{d}=\psi_{u}^{\dagger} \psi_{d}$, and evaluate its expectation value between proton and neutron states. In the naive quark model, the nucleon comprises three first-generation quarks arranged in a fully symmetric way with orbital angular momentum zero and spin 1/2. The expectation value is easily evaluated 7 :

$$
\left\langle p\left|V_{0}^{h}\right| n\right\rangle=1 .
$$

The result of unity implies the vector current normalization in the nucleon is unchanged from that in the quark Lagrangian. This, as we shall see, is as it should be and is a consequence of the conservation of the vector current. In contrast to this, consider the space component of the axial-vector part of the hadron current: $\mathbf{A}^{h}=i \bar{\psi}_{u} \gamma \gamma_{5} \psi_{d} \simeq-\boldsymbol{\sigma} \psi_{u}^{\dagger} \psi_{d}$ in a non-relativistic limit. Here $\boldsymbol{\sigma}$ is the Pauli spin matrix, which in its application here flips the quark spin. Thust

$$
\left\langle p\left|A_{3}^{h}\right| n\right\rangle=-\frac{5}{3}
$$

and the normalization of the axial current is indeed modified in a nucleon. The naive quark model value of $-5 / 3$ is larger than the experimental value of -1.26 obtained from the beta decay of the neutron. However, the nonrelativisitic treatment given here is not justifiable for quarks as light as $10 \mathrm{MeV}$. Typical relativistic quark calculations give values in the range -1.1 to -1.3 depending on the form of the quark wave functions used and the assumed masses of the quarks.

Since the quark model lacks the predictive precision required, we will follow the historic and more phenomenological approach of writing down a general Lagrangian using axioms and symmetry principles to limit the number of terms. For example, for the weak vector current, $V_{\mu}^{h}$, the most general form is constructed by our writing down all possible ways of making a Lorentz vector out of the vectors at our disposal: $p_{\mu}$, the incoming momentum of the nucleon; $p_{\mu}^{\prime}$, the outgoing momentum of the nucleon; and $\gamma_{\mu}$, the spin matrices. If it is further assumed that a nucleon in a nucleus undergoing a 
weak interaction can be treated as a free nucleon, which for the purposes of constructing interaction operators satisfies the Dirac equation, then the number of terms in the current is limited to three. This latter assumption is known as the impulse approximation. Similar arguments also limit the number of terms in the axial current to three. Both currents are written as

$$
\begin{aligned}
V_{\mu}^{h} & =i \bar{\psi}_{p}\left[g_{\mathrm{V}}\left(k^{2}\right) \gamma_{\mu}+\frac{g_{\mathrm{M}}\left(k^{2}\right)}{2 M} \sigma_{\mu \nu} k_{\nu}+i g_{\mathrm{S}}\left(k^{2}\right) k_{\mu}\right] \psi_{n} \\
A_{\mu}^{h} & =i \bar{\psi}_{p}\left[g_{\mathrm{A}}\left(k^{2}\right) \gamma_{\mu} \gamma_{5}+\frac{g_{\mathrm{T}}\left(k^{2}\right)}{2 M} \sigma_{\mu \nu} k_{\nu} \gamma_{5}+i g_{\mathrm{P}}\left(k^{2}\right) k_{\mu} \gamma_{5}\right] \psi_{n}
\end{aligned}
$$

where $k_{\mu}=\left(p-p^{\prime}\right)_{\mu}$ is the momentum transferred from hadrons to leptons and $\bar{\psi}_{p}$ and $\psi_{n}$ are field operators representing the proton and neutron respectively. The form factors $g_{\mathrm{V}}, g_{\mathrm{M}}, g_{\mathrm{S}}, g_{\mathrm{A}}, g_{\mathrm{T}}, g_{\mathrm{P}}$ are arbitrary functions of a Lorentz scalar constructed from the momenta $p^{\prime}$ and $p$. It is usual to choose this scalar to be $k^{2}$; any other scalar can be expressed in terms of $k^{2}$ and the nucleon mass, $M$. The values of these form factors in the zero-momentum transfer limit, $k^{2} \rightarrow 0$, are known as the vector, weak magnetism, induced scalar, axial vector, induced tensor and induced pseudoscalar coupling constants respectively.

The number of arbitrary functions in $V_{\mu}^{h}$ and $A_{\mu}^{h}$ can be reduced further by requiring that the currents be Hermitian and invariant under time reversal. These conditions lead to the requirement that $g_{\mathrm{V}}\left(k^{2}\right), g_{\mathrm{M}}\left(k^{2}\right), g_{\mathrm{A}}\left(k^{2}\right)$ and $g_{\mathrm{P}}\left(k^{2}\right)$ be real functions and that $g_{\mathrm{S}}\left(k^{2}\right)=g_{\mathrm{T}}\left(k^{2}\right)=0$. Further, the statement above that the normalization of the vector current is unchanged in going from the quark to the nucleon Lagrangian requires $g_{\mathrm{V}}\left(k^{2} \rightarrow 0\right)=1$. Additional constraints on the form factors follow from the conserved vector current (CVC) and the partially conserved axial-vector current (PCAC) hypotheses, which we discuss in later sections.

It is convenient in nuclear physics to make no distinction between protons and neutrons, but instead to use an isospin formalism. Let $\psi=\left(\psi_{p}, \psi_{n}\right)$ be an eightcomponent spinor operator representing the nucleon field, with $\psi_{p}$ and $\psi_{n}$ each being four-component field operators for the proton and neutron respectively. Further, introduce Pauli spin matrices, $\tau^{1}, \tau^{2}$ and $\tau^{3}$, forming the Cartesian components of a vector, $\boldsymbol{\tau}$, that are generalized in block form to act on $\psi$. For example, acting with $\tau^{3}$ on a pure proton state or a pure neutron state leads to

$$
\tau^{3}\left(\begin{array}{c}
\psi_{p} \\
0
\end{array}\right)=\left(\begin{array}{c}
\psi_{p} \\
0
\end{array}\right) ; \tau^{3}\left(\begin{array}{c}
0 \\
\psi_{n}
\end{array}\right)=-\left(\begin{array}{c}
0 \\
\psi_{n}
\end{array}\right) .
$$

These expressions serve to specify our isospin conventions. The currents in Eq. 10 in an isospin formulation are now written

$$
V_{\mu}^{h}=i \bar{\psi}\left[g_{\mathrm{V}}\left(k^{2}\right) \gamma_{\mu}+\frac{g_{\mathrm{M}}\left(k^{2}\right)}{2 M} \sigma_{\mu \nu} k_{\nu}+i g_{\mathrm{S}}\left(k^{2}\right) k_{\mu}\right] \frac{1}{2} \tau^{a} \psi
$$




$$
A_{\mu}^{h}=i \bar{\psi}\left[g_{\mathrm{A}}\left(k^{2}\right) \gamma_{\mu} \gamma_{5}+\frac{g_{\mathrm{T}}\left(k^{2}\right)}{2 M} \sigma_{\mu \nu} k_{\nu} \gamma_{5}+i g_{\mathrm{P}}\left(k^{2}\right) k_{\mu} \gamma_{5}\right] \frac{1}{2} \tau^{a} \psi
$$

where $\tau^{a}$ stands for $\tau^{1} \pm i \tau^{2}$ with a plus sign for $n \rightarrow p e^{-\bar{\nu}_{e}}$ and a minus sign for $p \rightarrow n e^{+} \nu_{e}$ charge-changing transitions.

Another symmetry of these currents to be investigated is their behaviour under charge conjugation, which interchanges particles and antiparticles, coupled with a $180^{\circ}$ rotation about the 2-axis in isospin space. This combination is called G-parity and is a symmetry of the strong interactions 8 :

$$
G=C \exp \left(i \pi T_{2}\right)
$$

Now, under charge conjugation, we havd

$$
C \psi_{i} C^{\dagger}=\psi_{i}^{\mathrm{C}} \equiv \gamma_{2} \psi_{i}^{\dagger}
$$

where $i=p, n$ and the representation of $C$ in terms of the Dirac matrix, $\gamma_{2}$, is specific to the metric we are using. Further, under an isospin rotation,

$$
\exp \left(i \pi T_{2}\right)\left(\begin{array}{c}
\psi_{p} \\
\psi_{n}
\end{array}\right)=-i \tau^{2}\left(\begin{array}{c}
\psi_{p} \\
\psi_{n}
\end{array}\right)=\left(\begin{array}{c}
-\psi_{n} \\
\psi_{p}
\end{array}\right) .
$$

Thus, combining these two operations, we have

$$
G \psi_{p} G^{\dagger}=-\psi_{n}^{\mathrm{C}} ; \quad G \psi_{n} G^{\dagger}=\psi_{p}^{\mathrm{C}}
$$

Applying these steps to the first term in the vector current, Eq. 10, we get

$$
\begin{aligned}
G V_{\mu}^{h} G^{\dagger} & =G \bar{\psi}_{p} G^{\dagger} \gamma_{\mu} G \psi_{n} G^{\dagger} \\
& =-i \psi_{n}^{\dagger \mathrm{C}} \gamma_{4} \gamma_{\mu} \psi_{p}^{\mathrm{C}} \\
& =-i\left(\psi_{n}^{\mathrm{C}}\right)_{\alpha}^{\dagger}\left(\gamma_{4} \gamma_{\mu}\right)_{\alpha \beta}\left(\psi_{p}^{\mathrm{C}}\right)_{\beta} \\
& =+i\left(\psi_{p}^{\mathrm{C}}\right)_{\beta}\left(\gamma_{4} \gamma_{\mu}\right)_{\beta \alpha}^{T}\left(\psi_{n}^{\mathrm{C}}\right)^{\dagger} \\
& =+i \psi_{p}^{\dagger} \gamma_{2} \gamma_{\mu}^{T} \gamma_{4}^{T} \gamma_{2} \psi_{n} \\
& =+i \bar{\psi}_{p} \gamma_{\mu} \psi_{n} \\
& =V_{\mu}^{h}
\end{aligned}
$$

where, in the third line, the expression is written out in component form and reordered in the fourth line, introducing a minus sign, because the fermion field operators anticommute. Thus, the first term in the vector current is invariant under G-parity transformations. Applying the same steps to the first term of the axial-current in Eq.10, we get 


$$
\begin{aligned}
G A_{\mu}^{h} G^{\dagger} & =i G \bar{\psi}_{p} G^{\dagger} \gamma_{\mu} \gamma_{5} G \psi_{n} G^{\dagger} \\
& =-A_{\mu}^{h} .
\end{aligned}
$$

We considered just the first terms of $V_{\mu}^{h}$ and $A_{\mu}^{h}$ because these are the only terms in the quark currents of the Standard Model. Weinberg 1 has termed currents with the standard quark-model transformation properties under G-parity as "first class", while currents with the opposite properties,

$$
\begin{aligned}
G V_{\mu}^{h(I I)} G^{\dagger} & =-V_{\mu}^{h(I I)} \\
G A_{\mu}^{h(I I)} G^{\dagger} & =A_{\mu}^{h(I I)},
\end{aligned}
$$

are termed "second class". Under this classification, the vector and weak magnetism terms of $V_{\mu}^{h}$ and the axial-vector and pseudoscalar terms of $A_{\mu}^{h}$ are first-class currents, while the induced scalar term of $V_{\mu}^{h}$ and the induced tensor term of $A_{\mu}^{h}$ are secondclass currents. To date, there is no unambiguous evidence for the presence of secondclass currents in nuclear beta decay, but it is nonetheless an interesting experimental challenge to place limits on the possible presence of these second-class currents.

\section{Vector Interaction}

\subsection{Conserved Vector Current Hypothesis}

Briefly, let us return to the quark Lagrangian of Eq.1 for the electromagnetic interaction and, in analogy with Eq.5, write down the electromagnetic current for the first family of quarks,

$$
V_{\mu}^{e m}=i \frac{2}{3} \bar{\psi}_{u} \gamma_{\mu} \psi_{u}-i \frac{1}{3} \bar{\psi}_{d} \gamma_{\mu} \psi_{d}
$$

in units of the coupling constant, $e$, which is the positron electric charge. Again, we can introduce isospin by defining a quark doublet spinor $\psi_{q}=\left(\psi_{u}, \psi_{d}\right)$ such that

$$
V_{\mu}^{e m}=i Q_{+} \bar{\psi}_{q} \gamma_{\mu} \psi_{q} I+i Q_{-} \bar{\psi}_{q} \gamma_{\mu} \psi_{q} \tau^{3}
$$

where $I$ is a unit matrix in isospin space; $Q_{+}$is the average quark charge, $Q_{+}=\frac{1}{2}\left(\frac{2}{3}-\frac{1}{3}\right)$, and $Q_{-}$the average difference, $Q_{-}=\frac{1}{2}\left(\frac{2}{3}+\frac{1}{3}\right)$. Note that the electromagnetic current has both isoscalar and isovector components. When the steps outlined in sect. 1.3 are repeated, the electromagnetic current between nucleons likewise exhibits isoscalar and isovector components and is written

$$
\begin{aligned}
V_{\mu}^{e m} & =i \bar{\psi}\left[F_{1}^{S}\left(k^{2}\right) \gamma_{\mu}+\frac{F_{2}^{S}\left(k^{2}\right)}{2 M} \sigma_{\mu \nu} k_{\nu}+i F_{3}^{S}\left(k^{2}\right) k_{\mu}\right] Q_{+} I \psi \\
& +i \bar{\psi}\left[F_{1}^{V}\left(k^{2}\right) \gamma_{\mu}+\frac{F_{2}^{V}\left(k^{2}\right)}{2 M} \sigma_{\mu \nu} k_{\nu}+i F_{3}^{V}\left(k^{2}\right) k_{\mu}\right] Q_{-} \tau^{3} \psi
\end{aligned}
$$


where now $Q_{+}$is the average nucleon charge and $Q_{-}$the average charge difference: $Q_{+}=Q_{-}=\frac{1}{2}$. Again, symmetry principles limit the number of terms to three for both the isoscalar and isovector currents. In terms of proton and neutron form factors, we identify

$$
\begin{aligned}
& F_{i}^{S}\left(k^{2}\right)=F_{i}^{p}\left(k^{2}\right)+F_{i}^{n}\left(k^{2}\right), \\
& F_{i}^{V}\left(k^{2}\right)=F_{i}^{p}\left(k^{2}\right)-F_{i}^{n}\left(k^{2}\right), \quad i=1,2,3 .
\end{aligned}
$$

Further, in going from quark to nucleon Lagrangians, the normalization of the vector current remains unchanged so that the first form factor $F_{1}$ in the $k^{2} \rightarrow 0$ limit simply specifies the charge of the nucleon in units of $e$ : viz. $F_{1}^{p}(0)=1, F_{1}^{n}(0)=0$, or equivalently $F_{1}^{S}(0)=1, F_{1}^{V}(0)=1$. The second form factor relates to the anomalous magnetic moment of the nucleon (in units of the nuclear magneton, $e /(2 M)$, with $\hbar=c=1)$ : viz. $F_{2}^{p}(0)=\mu_{p}-1=1.79$ and $F_{2}^{n}(0)=\mu_{n}=-1.91$, or equivalently $F_{2}^{S}(0)=-0.12$ and $F_{2}^{V}(0)=3.70$. Finally, the electromagnetic current, $V_{\mu}^{e m}$ is a conserved current: that is, $\partial_{\mu} V_{\mu}^{e m}=0$ or, as written in momentum space, $k_{\mu} V_{\mu}^{e m}=0$. This follows from the fact that the electromagnetic Lagrangian is invariant under the phase transformation $\psi \rightarrow \psi^{\prime} \overline{\bar{R}} e^{i e \Lambda} \psi$ and $\bar{\psi} \rightarrow \bar{\psi}^{\prime}=e^{-i e \Lambda} \psi$, where $\Lambda$ is a real constant. From Noether's theorem $\mathrm{\theta}$, for every transformation that leaves the Lagrangian invariant there is a corresponding conserved current, in this case the electromagnetic current. Evaluating $k_{\mu} V_{\mu}^{e m}$ term by term we have:

$$
\bar{\psi}\left(p^{\prime}\right) k_{\mu} \gamma_{\mu} \psi(p)=\bar{\psi}\left(p^{\prime}\right) \gamma_{\mu}\left(p_{\mu}-p_{\mu}^{\prime}\right) \psi(p)=i \bar{\psi}\left(p^{\prime}\right)(M-M) \psi(p)=0
$$

from the Dirac equation of a free nucleon; and $\sigma_{\mu \nu} k_{\mu} k_{\nu}$ summed over repeated indices is zero from the antisymmetry of $\sigma_{\mu \nu}$; while $k_{\mu}^{2}=k^{2} \neq 0$. Thus, current conservation requires that

$$
F_{3}\left(k^{2}\right)=0
$$

for both isoscalar and isovector currents.

It is noted that the isovector electromagnetic current, Eq.22, and the weak vector current, Eq. 12, are identical in structure with the isospin matrix $\tau^{3}$ in the former case replaced by the matrix $\tau^{a}$ in the latter case. Feynman and Gell-Mann 11 postulated that these currents are members of an isotriplet vector of current operators. A consequence of this hypothesis is that the weak vector current is also a conserved current. This is called the conserved vector current (CVC) hypothesis, from which it follows that

$$
\begin{aligned}
g_{\mathrm{V}}\left(k^{2}\right) & =F_{1}^{V}\left(k^{2}\right) \rightarrow 1 \quad \text { as } \quad k^{2} \rightarrow 0 \\
g_{\mathrm{M}}\left(k^{2}\right) & =F_{2}^{V}\left(k^{2}\right) \\
g_{\mathrm{S}}\left(k^{2}\right) & =F_{3}^{V}\left(k^{2}\right)=0 .
\end{aligned}
$$


Note that $g_{\mathrm{v}}(0)=1$ always, irrespective of the nucleus in which it might be experimentally measured. That is, the vector coupling constant is not renormalized in the nuclear medium. Likewise, the weak-magnetism form factor is uniquely related to the corresponding electromagnetic form factor: for beta decays between isospin analogues it relates to the anomalous isovector magnetic moment of the nuclear states in question, while for non-analogue transitions it relates to the corresponding isovector M1 gamma-decay rate.

So far, experimental tests of CVC have taken two forms: ( $i)$ showing $g_{\mathrm{V}}(0)$ is a constant irrespective of the nucleus in which it is measured, and (ii) showing $g_{\mathrm{M}}(0)=F_{2}^{V}(0)$ by measuring $g_{\mathrm{M}}(0)$ from either the lepton energy spectrum or an angular correlation in a beta-decay experiment and comparing it with $F_{2}^{V}(0)$ obtained from the lifetime and branching ratio for the analogous $\gamma$-transition. We discuss both tests in the following sections.

\subsection{Superallowed Fermi Transitions}

Precise measurements of the $\beta$-decay between nuclear states with $\left(J^{\pi}, T\right)=$ $\left(0^{+}, 1\right)$ provide the most straightforward yet demanding probe of the vector interaction in nuclei. Because the axial current cannot contribute in first order to transitions between spin-0 states, the experimental $f t$-value is related directly to the vector coupling constant, $G_{\mathrm{V}}=G_{\mathrm{F}} V_{u d}$ :

$$
f t\left(1+\delta_{R}\right)=\frac{K}{G_{\mathrm{V}}^{2}\left(1+\Delta_{\mathrm{R}}^{\mathrm{v}}\right)\left\langle M_{\mathrm{V}}\right\rangle^{2}},
$$

with

$$
\begin{aligned}
\left\langle M_{\mathrm{V}}\right\rangle^{2} & =2\left(1-\delta_{C}\right) \text { for } T=1 \\
K /(\hbar c)^{6} & =2 \pi^{3} \ln 2 \hbar /\left(m_{e} c^{2}\right)^{5}=(8120.271 \pm 0.012) \times 10^{-10} \mathrm{GeV}^{-4} \mathrm{~s}
\end{aligned}
$$

where the physical constants used to evaluate $K$ were taken from ref. 8 . Here $f$ is the statistical rate function, $t$ is the partial half-life for the transition, and $\left\langle M_{\mathrm{V}}\right\rangle$ is the Fermi matrix element. The radiative corrections, $\delta_{R}$ and $\Delta_{\mathrm{R}}^{\mathrm{v}}$, and the charge correction, $\delta_{C}$, are each of order 0.01 , with $\delta_{R}$ and particularly $\delta_{C}$ being dependent on nuclear structure. There are theoretical uncertainties introduced via these correction terms but they are at least another order of magnitude smaller, so Eq.27 provides an experimental technique for determining $G_{\mathrm{V}}$ to better than a part in a thousand.

To date, nine $0^{+} \rightarrow 0^{+}$transitions have been studied to this level of precision; they cover a wide range of nuclear masses from ${ }^{10} \mathrm{C}$, the lightest parent, to ${ }^{54} \mathrm{Co}$, the heaviest. The results have been used to test the constancy of $G_{\mathrm{V}}$, which is a prediction of CVC, and to derive the value of $V_{u d}$, the up-down element in the Cabibbo-KobayashiMaskawa quark-mixing matrix. A unitarity test on the matrix, made possible by these results, is a fundamental measure of the adequacy of the three-generation Standard Model. 
Table 2. Experimental results $\left(Q_{E C}, t_{1 / 2}\right.$ and branching ratio, $\left.R\right)$ and calculated corrections $\left(P_{E C}, \delta_{C}\right.$ and $\left.\delta_{R}\right)$ for $0^{+} \rightarrow 0^{+}$transitions.

\begin{tabular}{ccccccccc}
\hline & $\begin{array}{c}Q_{E C} \\
(\mathrm{keV})\end{array}$ & $\begin{array}{c}t_{1 / 2} \\
(\mathrm{~ms})\end{array}$ & $\begin{array}{c}R \\
(\%)\end{array}$ & $\begin{array}{c}P_{E C} \\
(\%)\end{array}$ & $\begin{array}{c}f t \\
(\mathrm{~s})\end{array}$ & $\begin{array}{c}\delta_{C} \\
(\%)\end{array}$ & $\begin{array}{c}\delta_{R} \\
(\%)\end{array}$ & $\begin{array}{c}\mathcal{F} t \\
(\mathrm{~s})\end{array}$ \\
\hline${ }^{10} \mathrm{C}$ & $1907.77(9)$ & $19290(12)$ & $1.4638(22)$ & 0.296 & $3040.1(51)$ & $0.18(4)$ & $1.30(4)$ & $3074.0(54)$ \\
${ }^{14} \mathrm{O}$ & $2830.51(22)$ & $70603(18)$ & $99.336(10)$ & 0.087 & $3038.1(18)$ & $0.23(4)$ & $1.26(5)$ & $3069.2(28)$ \\
${ }^{26 m} \mathrm{Al}$ & $4232.42(35)$ & $6344.9(19)$ & $\geq 99.97$ & 0.083 & $3035.8(17)$ & $0.31(4)$ & $1.45(2)$ & $3070.2(23)$ \\
${ }^{34} \mathrm{Cl}$ & $5491.71(22)$ & $1525.76(88)$ & $\geq 99.988$ & 0.078 & $3048.4(19)$ & $0.54(4)$ & $1.33(3)$ & $3072.1(25)$ \\
${ }^{38 m} \mathrm{~K}$ & $6043.76(56)$ & $923.95(64)$ & $\geq 99.998$ & 0.082 & $3047.9(26)$ & $0.56(4)$ & $1.33(4)$ & $3071.1(32)$ \\
${ }^{42} \mathrm{Sc}$ & $6425.58(28)$ & $680.72(26)$ & $99.9941(14)$ & 0.095 & $3045.1(14)$ & $0.36(4)$ & $1.47(5)$ & $3078.7(24)$ \\
${ }^{46} \mathrm{~V}$ & $7050.63(69)$ & $422.51(11)$ & $99.9848(13)$ & 0.096 & $3044.6(18)$ & $0.37(4)$ & $1.40(6)$ & $3075.8(28)$ \\
${ }^{50} \mathrm{Mn}$ & $7632.39(28)$ & $283.25(14)$ & $99.942(3)$ & 0.100 & $3043.7(16)$ & $0.41(4)$ & $1.40(7)$ & $3073.8(28)$ \\
${ }^{54} \mathrm{Co}$ & $8242.56(28)$ & $193.270(63)$ & $99.9955(6)$ & 0.104 & $3045.8(11)$ & $0.50(4)$ & $1.39(7)$ & $3073.1(28)$ \\
& & & & & & \multicolumn{2}{c}{ Average, $\overline{\mathcal{F} t}$} & $3073.1(11)$ \\
& & & & & & & $\chi^{2} /(N-1)$ & 1.34 \\
\hline
\end{tabular}

\subsubsection{Experiments}

The $f t$ value that characterizes a particular $\beta$-transition is determined by three measured parameters: the transition energy, $Q_{E C}$, which is used in calculating the statistical rate function, $f$; the half-life, $t_{1 / 2}$, of the $\beta$-emitter and the branching ratio, $R$, for the transition of interest, which together yield the partial half-life, $t$. World data have been surveyed recently 12 for eight of the best-known superallowed emitters: ${ }^{14} \mathrm{O},{ }^{26 m} \mathrm{Al},{ }^{34} \mathrm{Cl}$, ${ }^{38 m} \mathrm{~K},{ }^{42} \mathrm{Sc},{ }^{46} \mathrm{~V},{ }^{50} \mathrm{Mn}$ and ${ }^{54} \mathrm{Co}$. The results given in Table 2 are drawn from that survey, augmented by data on ${ }^{10} \mathrm{C}$ decav $13,14,15,16,17,28,19,20,21$ and by several other recent measurements of significance.22, 23, 24, 25, 26, 27, 28, 29

The experimental uncertainties quoted for the measured decay energies (typically 1 part in 20,000), half-lives (1 part in 3,000) and branching ratios (1 part in 70,000) are at the limits of what is currently possible. Even though such quantities are frequently measured in nuclear physics, the extreme demands on precision in these studies have led to experimental techniques highly refined for the purpose. Several examples, briefly described, will illustrate the point.

2.2.1.1 Decay energies. The nine superallowed $\beta$-emitters listed in Table 2 populate daughters with stable ground states. As a result, reaction $Q$-value measurements, which lend themselves to experimental precision, can be used to determine decay energies instead of direct measurements of positron end-point energies, which are frought with precision-limiting difficulties. For example, in the case of ${ }^{26 m} \mathrm{Al} \rightarrow{ }^{26} \mathrm{Mg}$, the decay energy, $Q_{E C}$, has been measured by three different routes:

(i) ${ }^{26} \mathrm{Mg}(p, n){ }^{26 m} \mathrm{Al}$. The threshold energy for this reaction was determined 26 by detection of the ${ }^{26 m} \mathrm{Al}$ activity produced as a function of proton energy, with a ${ }^{26} \mathrm{Mg}$ target located in the focal plane of a magnetic spectrometer. The energy of the proton 
beam was calibrated by comparison with a ${ }^{133} \mathrm{Cs}^{+}$beam accelerated through a voltage difference $V$ to pass along the same path in the spectrometer. The value of $V$ was then compared via a resistive divider with a one-volt standard. The measured $Q$ value, $Q_{p n}$, derived from the observed threshold energy, is related to $Q_{E C}$ by:

$$
Q_{E C}=-Q_{p n}-782.354 \mathrm{keV} .
$$

(ii) ${ }^{25} \mathrm{Mg}(p, \gamma){ }^{26 m} \mathrm{Al}$ and ${ }^{25} \mathrm{Mg}(n, \gamma){ }^{26} \mathrm{Mg}$. For the first reaction, proton energies at four resonances were established 22 by comparison with known resonances in ${ }^{27} \mathrm{Al}(p, \gamma){ }^{28} \mathrm{Si}$. For the second, thermal neutrons were used, with $\gamma$-ray energies being determined 22 by comparison with known calibration lines. The measured $Q$ values yield $Q_{E C}$ through the relation:

$$
Q_{E C}=Q_{n \gamma}-Q_{p \gamma}-782.354 \mathrm{keV} .
$$

Though comparable uncertainties are claimed as for method (i), it is evident that this method is less direct and involves a variety of secondary calibration standards.

(iii) ${ }^{26} \mathrm{Mg}\left({ }^{3} \mathrm{He}, t\right){ }^{26} \mathrm{Al}(1058 \mathrm{keV})$. This reaction was observed 30 in conjunction with ${ }^{14} \mathrm{~N}\left({ }^{3} \mathrm{He}, t\right){ }^{14} \mathrm{O}$ from a composite target in which ${ }^{14} \mathrm{~N}$ had been implanted into ${ }^{26} \mathrm{Mg}$. The target assembly was biased periodically to $\sim 80 \mathrm{kV}$, thus permitting the two triton groups corresponding to the ${ }^{14} \mathrm{O}$ ground state and the $1058 \mathrm{keV}$ state of ${ }^{26} \mathrm{Al}$ alternately to follow the same path through a magnetic spectrometer. The voltage at which they perfectly overlapped corresponds to the $Q$-value difference, $\Delta Q$, between the two reactions. The energy difference between the $1058 \mathrm{keV}$ state and the ${ }^{26} \mathrm{Al} 0^{+}$ isomer was determined 31 from the relevant $\gamma$-ray energy, $E_{\gamma_{1}}$. Similarly, the ground state of ${ }^{14} \mathrm{~N}$ can be related to the $0^{+}(T=1)$ excited state by a $\gamma$ ray of energy, $E_{\gamma_{2}}$. Thus,

$$
Q_{E C}(\mathrm{~A}=26)-Q_{E C}(\mathrm{~A}=14)=E_{\gamma_{2}}-E_{\gamma_{1}}-\Delta Q .
$$

This method, too, depends on secondary calibration standards but it provides valuable interconnections betwen pairs of superallowed $0^{+} \rightarrow 0^{+}$transition $Q_{E C}$ values.

2.2.1.2 Half-lives. To achieve the required precision, isotopic purity of the sample must be assured. Currently, all emitters except ${ }^{14} \mathrm{O}$ and ${ }^{10} \mathrm{C}$ have been measured ${ }^{24}$ with an on-line isotope separator. Typically, thousands of multi-scaled spectra are acquired from successive samples observed in a large-solid-angle, high-efficiency, lowbackground gas proportional counter; the counting electronics have a well-defined, nonextendable dead time. The best analysis procedure uses modified Poisson variances and is based on a simultaneous fit to up to 500 decay curves with the same half-life but with individual intensity parameters for each decay curve. This procedure is checked 24 with hypothetical data, computer-generated to simulate closely the experimental counting conditions, and has been proven correct at the $0.01 \%$ level.

2.2.1.3 Branching ratios. In most cases, the superallowed transition dominates $(\geq 99 \%)$ the decay of the parent nucleus and is best determined via measurement 
of the other decay branches, their branching ratios being subtracted from $100 \%$. For these other branches, precision is not as important as completeness; therefore special techniques have been developed (e.g. ref.29) to observe $\beta$-delayed $\gamma$ rays from branches as weak as a few parts per million. For the only case in which the superallowed transition is itself weak - the decay of ${ }^{10} \mathrm{C}$ - the technique used 18 is to compare the intensity of $\beta$-delayed $\gamma$ rays in ${ }^{10} \mathrm{~B}$, produced via the reaction ${ }^{10} \mathrm{~B}(p, n){ }^{10} \mathrm{C}\left(\beta^{+}\right){ }^{10} \mathrm{~B}^{*}$, with the same $\gamma$ rays produced via ${ }^{10} \mathrm{~B}\left(p, p^{\prime}\right){ }^{10} \mathrm{~B}^{*}$; for added precision, a large $\gamma$-ray array 21 has now been used to observe these $\gamma$ rays in alternating off-line, on-line measurements.

\subsubsection{The $f t$ values}

The statistical rate function, $f$, is defined by

$$
f=\int_{1}^{W_{0}} p W\left(W_{0}-W\right)^{2} F(Z, W) C(W) \mathrm{d} W
$$

where $W$ and $p$ are the electron energy and momentum, respectively, in electron restmass units, and $W_{0}$ is the maximum $\beta$-energy, which is related directly to the measured $Q_{E C}$ via the equation $W_{0}+1=Q_{E C} /\left(m_{e} c^{2}\right)$. The quantity $F(Z, W)$ is known as the Fermi function and $C(W)$ the shape correction factor 32 . The latter has a value close to unity for most values of $W$, its departure from unity reflecting the influence of screening by atomic electrons, the dependence of the nuclear matrix elements on $\mathrm{W}$, and the presence of second-forbidden matrix elements. Values of the statistical rate function have been computed for each superallowed transition following the techniques

described in refs.1233. Each result has an associated uncertainty reflecting the experimental uncertainty in the $Q_{E C}$ value used in the calculation.

Next, the partial half-life for each superallowed emitter must be obtained from its total half-life and branching ratio according to the formula

$$
t=t_{1 / 2}\left(1+P_{E C}\right) / R,
$$

where $P_{E C}$ is the calculated electron-capture probability. Table 2 lists the $P_{E C}$ values calculated by the method described in ref.12, which employs exact bound-state electron wave functions. Though the calculated values of $P_{E C}$ are small in all cases, the correction is comparable to the experimental uncertainties on $t_{1 / 2}$ and $R$, and must be properly accounted for.

Finally, the $f t$-values that incorporate all experimental uncertainties are listed for the nine emitters in Table 2. They incorporate the complete set of world data and form the experimental basis for the fundamental tests that follow.

\subsubsection{Theoretical corrections}

In a nucleus, the charged particles involved in $\beta$ decay interact with an external electromagnetic field, which leads to effects of order $(Z \alpha)^{m}, m=1,2 \ldots$, where $Z$ is the atomic 
number of the daughter nucleus and $\alpha$ is the fine-structure constant. For the electron (or positron), these effects are accounted for in the calculation of $f$ (see Eq.27), which uses 12.33 exact solutions of the Dirac equation for electrons in the field of a modelled nuclear charge distribution. For the proton, these effects are reflected in the breaking of nuclear isospin symmetry and a resulting correction, $\delta_{C}$, to the Fermi matrix element.

There are also effects that arise from interactions between the charged particles themselves. These are of order $Z^{m} \alpha^{n}$, with $m<n$, and lead to the radiative corrections, $\delta_{R}$ and $\Delta_{\mathrm{R}}^{\mathrm{v}}$. By convention, the former incorporates those corrections that are nuclearstructure dependent, the latter, those that are not.

2.2.3.1 Charge correction, $\delta_{C}$. Both Coulomb and charge-dependent nuclear forces destroy isospin symmetry between the initial and final states in superallowed $\beta$-decay. The consequences are twofold: there are different degrees of configuration mixing in the two states, and, because their binding energies are not identical, their radial wave functions differ. Calculation 122.293 .34 accommodate both effects by splitting $\delta_{C}$ into two components, $\delta_{C_{1}}$ and $\delta_{C_{2}}$, to account for configuration mixing and radial mismatch, respectively. In all cases $\delta_{C_{2}}>\delta_{C_{1}}$.

To establish $\delta_{C_{1}}$, shell-model wave functions were calculated with appropriate nucleon-nucleon interactions modified to include: $(i)$ two-body Coulomb terms in the proton-proton part of the Hamiltonian, $(i i)$ a $2 \%$ increase in proton-neutron elements (justified by the charge dependence observed in nucleon-nucleon scattering data), and (iii) one-body elements determined from the single-particle energies of closed-coreplus-proton and -neutron nuclei. These basic elements were then adjusted to reproduce the $b$ - and $c$-coefficients of the isobaric-multiplet mass equation that describes the multiplet involved in the superallowed transition. The results have also been tested independently by measurements 29 , in some of the same nuclei, of weak non-analogue $0^{+} \rightarrow 0^{+}$transitions, which are sensitive to the same effects.

The larger of the two components, $\delta_{C_{2}}$, has been calculated from two different approaches. Ond 35 describes the proton in the parent nucleus and the neutron in the daughter nucleus by full parentage expansions in terms of Woods-Saxon wave functions suitably matched to the experimentally known separation energies. The other $\$$ employs a self-consistent Hartree-Fock calculation in which the overall strength of the central potential was adjusted so that the single-particle eigenvalues agree with the experimental separation energies. The former approach leads to values for $\delta_{C_{2}}$ that are consistently larger than those from the latter.

In Table 2 , we list $\delta_{C}\left(=\delta_{C_{1}}+\delta_{C_{2}}\right)$ for all nine transitions. The values for $\delta_{C_{1}}$ were taken from ref.12 updated by new results in ref.29; those for $\delta_{C_{2}}$ are the unweighted averages from the results of refs. 34 35. "Statistical" uncertainties, reflecting the scatter of the calculated values, were derived in ref. 12 and have been adopted here.

2.2.3.2 Radiative corrections, $\delta_{R}$ and $\Delta_{\mathrm{R}}^{\mathrm{v}}$. In the context of Standard-Model tests, we need consider only radiative-correction terms that distinguish semi-leptonic nuclear $\beta$-decay from purely leptonic $\mu$-decay 36 . By grouping them according to whether they are nuclear-structure dependent $\left(\delta_{R}\right)$ or not $\left(\Delta_{\mathrm{R}}^{\mathrm{V}}\right)$, we write 37 : 


$$
\begin{aligned}
\delta_{R} & =\frac{\alpha}{2 \pi}\left[\bar{g}\left(E_{m}\right)+\delta_{2}+\delta_{3}+2 C_{N S}\right] \\
\Delta_{\mathrm{R}}^{\mathrm{v}} & =\frac{\alpha}{2 \pi}\left[4 \ln \left(m_{\mathrm{z}} / m_{p}\right)+\ln \left(m_{p} / m_{\mathrm{A}}\right)+2 C_{\mathrm{Born}}\right]+\ldots,
\end{aligned}
$$

where, for clarity, only leading-order terms have been presented for $\Delta_{\mathrm{R}}^{\mathrm{V}}$. In these equations, $E_{m}$ is the maximum electron energy in the $\beta$-decay, $m_{p}$ and $m_{\mathrm{z}}$ are the masses of the proton and $Z$-boson, and $m_{\mathrm{A}}$ is a mass parameter associated with the $A_{1}$-meson but, in this application, is allowed the range $400 \leq m_{\mathrm{A}} \leq 1600 \mathrm{MeV}$. The function $g\left(E, E_{m}\right)$ was first defined by Sirlin 38 as part of the order- $\alpha$ universal photonic contribution arising from the weak vector current; it is here averaged over the electron spectrum to give $\bar{g}\left(E_{m}\right)$. The effects of order- $Z \alpha^{2}$ and $-Z^{2} \alpha^{3}$ have recently been definitively calculated ${ }^{39}$ and are incorporated as $\delta_{2}$ and $\delta_{3}$, respectively. Finally, the terms $C_{\text {Born }}$ and $C_{N S}$ arise from the order- $\alpha$ axial-vector photonic contributions: the former accounts for single-nucleon contributions, while the latter covers two-nucleon contributions and is consequently dependent on nuclear structure. We adopt here the calculations of Townet 40 .

Built from these components (see Eq. 34), calculated values for $\delta_{R}$ are listed in Table 2. The largest component, $\bar{g}\left(E_{m}\right)$, is responsible for approximately $1 \%$ in each case, $\delta_{2}$ and $\delta_{3}$ together account for less than $0.5 \%$, and the effects of $C_{N S}$ range from $-0.3 \%$ to $+0.1 \%$. The calculated value for $\Delta_{\mathrm{R}}^{\mathrm{V}}$ is

$$
\Delta_{\mathrm{R}}^{\mathrm{v}}=(2.46 \pm 0.09) \% .
$$

In this case, the leading logarithic term dominates, with a contribution of $2.1 \%$. The remainder arises from $C_{\mathrm{Born}}(0.20 \%)$ and higher order terms not explicitly listed in Eq. 35. The second term in the equation effectively establishes the quoted uncertainty, which reflects the range of values allowed for $m_{\mathrm{A}}$.

\subsubsection{Implications for the Standard Model}

It is now convenient to define a "corrected" $\mathcal{F} t$ value, which is related to the conventional $f t$ value via:

$$
\mathcal{F} t=f t\left(1+\delta_{R}\right)\left(1-\delta_{C}\right) .
$$

As a comparison with Eq.27 makes clear, all nuclear-structure-dependent effects should have been removed from the $\mathcal{F} t$ values. It is the consistency of the determined $\mathcal{F} t$ values over a wide variety of superallowed $0^{+} \rightarrow 0^{+}$transitions that constitutes a demanding test of CVC.

Table 2 contains all requisite experimental and calculated information for the determination of $\mathcal{F} t$ values, and the results for all nine transitions appear in the last

column of that table together with their weighted average, $\overline{\mathcal{F} t}$, and scaled "statistical" 


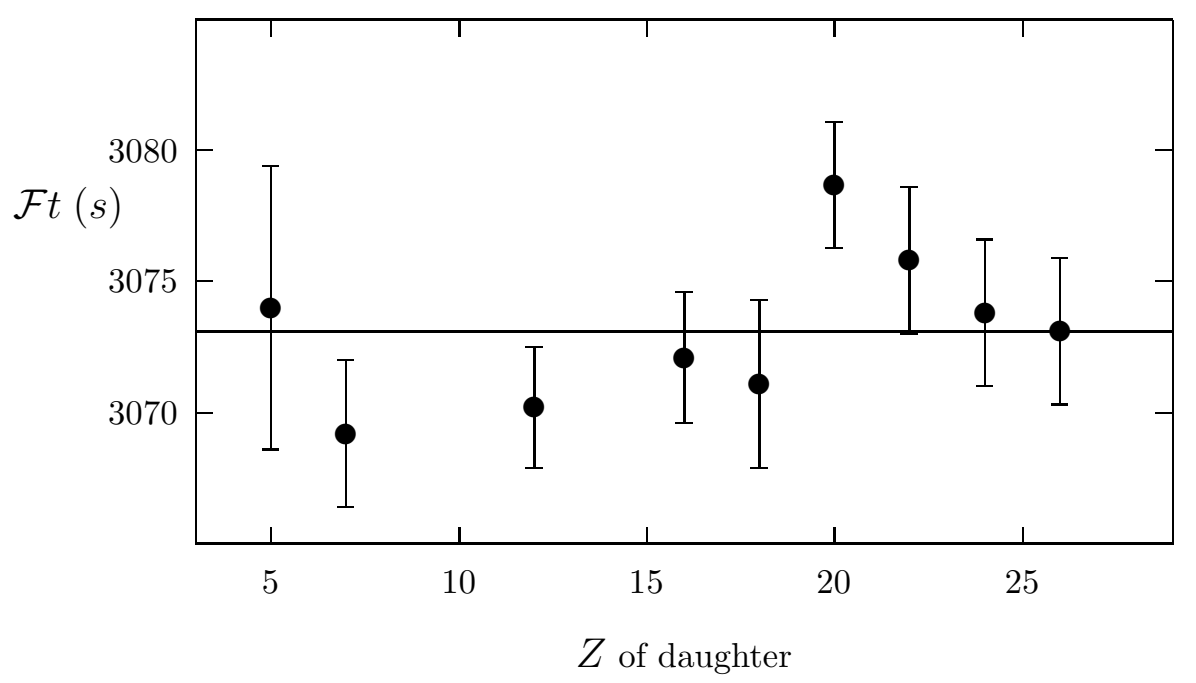

Fig. 1. $\mathcal{F} t$ values for the nine precision data and the best least squares one-parameter fit

uncertainty. The same results are displayed in Fig.11. There is no statistically significant evidence of inconsistencies in the data, thus verifying the expectation of CVC at the level of $4 \times 10^{-4}$, the fractional uncertainty quoted on the average $\mathcal{F}$-value.

With the consistency of the measured $\mathcal{F}$ t-values established, we turn to the evaluation of $V_{u d}$ and the unitarity test of the CKM matrix. The up-down quark mixing matrix element is given by

$$
\begin{aligned}
V_{u d}^{2} & =G_{\mathrm{V}}^{2} / G_{\mathrm{F}}^{2} \\
& =\frac{K}{2 G_{\mathrm{F}}^{2}\left(1+\Delta_{\mathrm{R}}^{\mathrm{v}}\right) \overline{\mathcal{F} t}} .
\end{aligned}
$$

In using the average $\overline{\mathcal{F} t}$ value from Table 2 in Eq. 38 , it is important now for us to include in the $\overline{\mathcal{F}}$-value uncertainty the effects of a "systematic" uncertainty in the charge correction, $\delta_{C}$, as well as the "statistical" uncertainty already described. The "systematic" uncertainty of $\pm 0.08 \%$ arises from systematic differences between two independent model calculation $\$ 35$ of $\delta_{C_{2}}$, and has been elaborated on in ref. 12 . The result is

$$
\overline{\mathcal{F} t}=3073.1 \pm 3.0 \mathrm{~s}
$$

Thus, with 


$$
G_{\mathrm{F}} /(\hbar c)^{3}=(1.16639 \pm 0.00002) \times 10^{-5} \mathrm{GeV}^{-2}
$$

from ref.6 and $\Delta_{\mathrm{R}}^{\mathrm{v}}$ from Eq. 36 , we obtain

$$
V_{u d}=0.9736 \pm 0.0006
$$

The quoted uncertainty is dominated by uncertainties in the theoretical corrections, $\Delta_{\mathrm{R}}^{\mathrm{v}}$ and $\delta_{C}$; experimental errors on the input data for the $\overline{\mathcal{F} t}$-value contribute less than \pm 0.0002 to the uncertainty in Eq. 41 .

This result for $V_{u d}$ leads to the most demanding test yet available of the unitarity of the Cabibbo-Kobayashi-Maskawa matrix, in which

$$
\left|V_{u d}\right|^{2}+\left|V_{u s}\right|^{2}+\left|V_{u b}\right|^{2}=1
$$

must be satisfied if there are three generations of quarks prescribed by the Standard Model. Evidently, $V_{u d}$ is by far the largest of the three matrix elements and its uncertainty the most critical. The value of $V_{u s}$ is taken to be $\left|V_{u s}\right|=0.2205 \pm 0.0018$, the average of two results, one from the analysis of $K_{e 3}$ decays, the other from hyperon decays. Finally, $V_{u b}$ can be derived, with substantial uncertainty, from the semi-leptonic decay of $B$ mesons, but is sufficiently small6, at $0.0032 \pm 0.0009$, as to be negligible in the unitarity test. The result,

$$
\left|V_{u d}\right|^{2}+\left|V_{u s}\right|^{2}+\left|V_{u b}\right|^{2}=0.9965 \pm 0.0015
$$

differs from unitarity at the $98 \%$ confidence level.

The significance of this apparent non-unitarity is still being debated. Taken at face value it indicates the need for some extension to the three-generation Standard Model (see sect.5). However, it may also reflect some undiagnosed inadequacy in the evaluation of $\left|V_{u s}\right| \# 3$ or $\left|V_{u d}\right|$. For example, it has been proposed $\$ 2$ that the nuclearmodel-dependent calculations of $\delta_{C}$ should be replaced by an empirical approach in which the uncorrected data are fitted by a smooth $Z$-dependent function. Extrapolation of this function to $Z=0$ would yield the recommended value of $\mathcal{F} t$. With the data in Table 2, a variation of this approach is to allow a $Z$-dependent fit of the nine $\mathcal{F} t$ values: doing so with linear $Z$ dependence does lower the reduced $\chi^{2}$ somewhat (from 1.34 to 1.04$)$. The result is $\mathcal{F} t(Z=0)=3068.1 \pm 3.1$, which yields a new unitarity sum (Eq.43) of $0.9980 \pm 0.0015$. It must be emphasized, however, that although this approach does yield a less provocative result, there is no substantive explanation for any correction not already accounted for in $\delta_{C}$ and $\delta_{R}$, and there is no statistically significant indication from the data that one is required.

$\overline{\# 3} \mathrm{~A}$ larger value is recommended in the analysis of ref 4 


\subsection{Experimental Tests of CVC in Weak Magnetism Terms}

The conserved vector current hypothesis, besides requiring that $g_{\mathrm{V}}(0)$ is constant $(=1)$, as verified in superallowed transitions, also predicts that the weak magnetism form factor is uniquely related to the corresponding electromagnetic form factor. In this section we will discuss experimental tests of this latter prediction, concentrating our attention principally on the $\left(J^{\pi}, T=1^{+}, 1\right) \rightarrow\left(0^{+}, 0\right)$ allowed axial-vector mirror $\beta$-transitions in the $A=12$ nuclei: ${ }^{12} \mathrm{~B} \rightarrow{ }^{12} \mathrm{C}+e^{-}+\bar{\nu}_{e}$ and ${ }^{12} \mathrm{~N} \rightarrow{ }^{12} \mathrm{C}+e^{+}+\nu_{e}$. For oriented parent nuclei, the beta-decay rates (upper sign for ${ }^{12} \mathrm{~N}$ decay and lower sign for ${ }^{12} \mathrm{~B}$ decay) are given by 43

$$
\begin{aligned}
d^{3} \Gamma\left(e^{ \pm}\right)= & \frac{1}{\hbar} \frac{G_{\mathrm{F}}^{2}}{4 \pi^{4}} V_{u d}^{2}\left|F_{A}^{ \pm}(0)\right|^{2} F_{ \pm}(Z, W) p W\left(W_{0}-W\right)^{2}\left(1+\eta_{ \pm}+a_{ \pm} W\right) d W d \Omega_{e} \\
& \times\left[1 \pm\left(h_{1}-h_{-1}\right)\left(1+\alpha_{ \pm} W\right) P_{1}\left(\cos \theta_{e}\right)+\left(1-3 h_{0}\right) \alpha_{ \pm} W P_{2}\left(\cos \theta_{e}\right)\right],(44)
\end{aligned}
$$

where $F_{ \pm}(Z, W)$ is the Fermi function for the $e^{ \pm}$decays, $p$ and $W$ are the electron momentum and total energy in electron rest-mass units; and $W_{0}$ is the maximum electron energy. The shape correction factor, $C(W)$, has been explicitly written as $\left(1+\eta_{ \pm}+a_{ \pm} W\right)$. In addition, $h_{1}, h_{-1}, h_{0}$ are the populations of the $m=1,-1,0$ states of ${ }^{12} \mathrm{~N}$ or ${ }^{12} \mathrm{~B}$, normalized such that $h_{1}+h_{-1}+h_{0}=1 ; \cos \theta_{e}=\hat{p} \cdot \hat{z}$, and $\Gamma=\ln 2 / t$, where $t$ is the decay half-life. The coefficients $\eta_{ \pm}, a_{ \pm}$and $\alpha_{ \pm}$are expressed, in the formalism of Hwang and Primakofft3, in terms of nuclear form factors, $F$ :

$$
\begin{aligned}
& \eta_{ \pm}=\frac{W_{0}^{ \pm}}{3 M}\left( \pm 2 \frac{F_{M}^{ \pm}(0)}{F_{A}^{ \pm}(0)}+\frac{F_{E}^{ \pm}(0)}{F_{A}^{ \pm}(0)}\right) \\
& a_{ \pm}=\mp \frac{4}{3 M} \frac{F_{M}^{ \pm}(0)}{F_{A}^{ \pm}(0)}+\delta_{ \pm}(\alpha Z) \\
& \alpha_{ \pm}=\frac{1}{3 M}\left(\mp \frac{F_{M}^{ \pm}(0)}{F_{A}^{ \pm}(0)}-\frac{F_{E}^{ \pm}(0)}{F_{A}^{ \pm}(0)}\right),
\end{aligned}
$$

where $M$ is the nucleon mass and $\delta_{ \pm}(\alpha Z)$ are radiative corrections calculated to be $\delta_{-}=1.31 \mathrm{GeV}^{-1}$ and $\delta_{+}=2.69 \mathrm{GeV}^{-1}$ by Behrens and Szybisz 4 . These nuclear form factors originate in an elementary-particle treatment 13 of the decays in which no explicit consideration is given to the dependence of the wavefunctions on the coordinates of the constituent nucleons, nor is the weak current itself decomposed into contributions from individual nucleons. Rather, the details of nuclear structure are buried in the nuclear form factors, which have then to be determined from experiment.

With sufficient data, this approach provides a treatment that is essentially independent of nuclear models and permits analyses to explore the consequences of symmetry properties and conservation hypotheses. But with insufficient data, some of the form factors have to be obtained from other sources, the most common being to appeal 
to the impulse approximation. In the latter case, the starting point is the single-nucleon currents of Eq. 12 reduced to their nonrelativistic form to yield one-body operators, the matrix elements of which are evaluated with many-body wavefunctions. The coupling constants of Eq.12, $g\left(k^{2}\right) \simeq g(0)$, are determined from data on the free proton and neutron and treated as constants, unchanged from nucleus to nucleus. The connection with the nuclear form factors of the elementary-particle approach is given by 43.45

$$
\begin{aligned}
& \sqrt{2} F_{M}^{ \pm}(0) \rightarrow-\left(1+g_{\mathrm{M}}\right)\langle\boldsymbol{\sigma}\rangle-\langle\mathbf{L}\rangle \\
& \sqrt{2} F_{A}^{ \pm}(0) \rightarrow-\left(g_{\mathrm{A}} \mp \frac{W_{0}^{ \pm}}{2 M} g_{\mathrm{T}}\right)\langle\boldsymbol{\sigma}\rangle \\
& \sqrt{2} F_{E}^{ \pm}(0) \rightarrow-\left(g_{\mathrm{A}} \pm g_{\mathrm{T}}\right)\langle\boldsymbol{\sigma}\rangle-2 g_{\mathrm{A}}\langle i \mathbf{r}(\boldsymbol{\sigma} \cdot \boldsymbol{p})\rangle,
\end{aligned}
$$

where, for example, $\langle\boldsymbol{\sigma}\rangle$ is the Gamow-Teller matrix element defined more completely below in Eq.94.

The transition rate for the analogous gamma decay, ${ }^{12} \mathrm{C}^{*}\left(1^{+}, 1\right) \rightarrow{ }^{12} \mathrm{C}\left(0^{+}, 0\right)$, is specified by

$$
\Gamma\left({ }^{12} \mathrm{C}^{*} \rightarrow{ }^{12} \mathrm{C}\right)=\frac{\alpha}{3} \frac{E_{\gamma}^{3}}{M^{2}}|\sqrt{2} \mu(0)|^{2},
$$

where $\alpha$ is the fine-structure constant, $E_{\gamma}$ the photon decay energy and $\mu(0)$ the isovector magnetic nuclear form factor, which, in the nonrelativistic limit, connects to impulse-approximation quantities 43,45 via

$$
\sqrt{2} \mu(0) \rightarrow-\left(\mu_{p}-\mu_{n}+1\right)\langle\boldsymbol{\sigma}\rangle-\langle\mathbf{L}\rangle
$$

with $\mu_{p}$ and $\mu_{n}$ the proton and neutron nucleon magnetic moments respectively. The test of CVC consists of the verification of the relation

$$
F_{M}^{ \pm}(0)=\sqrt{2} \mu(0)
$$

The $\sqrt{2}$ displayed in Eq. 49 occurs because the isospin operator for beta decay is $\left(\tau^{1} \pm\right.$ $i \tau^{2}$ ), whereas the isospin spherical tensor operator that relates to the $\tau_{3}$ operator of gamma decay through an isospin rotation is $\left(\tau^{1} \pm i \tau^{2}\right) / \sqrt{2}$.

The weighted average of six measurements 4 of $\Gamma_{\gamma}$ that use the technique of nuclear resonance fluorescence (after reevaluation with currently accepted branching ratios) is $\Gamma_{\gamma}=40.4 \pm 2.0 \mathrm{eV}$. The three most recent measurements of $\Gamma_{\gamma}$ that use ${ }^{12} \mathrm{C}\left(e, e^{\prime}\right)$ yield $\Gamma_{\gamma}=37.0 \pm 1.1 \mathrm{eV}$ (ref. 4 ), $35.74 \pm 0.86 \mathrm{eV}$ (ref. 4 ) and $38.5 \pm 0.8 \mathrm{eV}$ (ref. 18 ). Following de Braeckeleer 49 , we adopt the value $\Gamma_{\gamma}=38.8 \pm 1.6 \mathrm{eV}$, which is an average of the combined data with an error chosen to make the two data sets consistent with each other. There is a small correction 50 due to isospin mixing of the ${ }^{12} \mathrm{C}^{*}\left(1^{+}, 1\right)$ state at $15.11 \mathrm{MeV}$ with the $\left(1^{+}, 0\right)$ state at $12.71 \mathrm{MeV}$, but its magnitude is smaller 
than the stated error on $\Gamma_{\gamma}$ so we consider it to be included in that estimate. Thus, from Eqs. 47 and 49 we obtain as a prediction of CVC:

$$
F_{M}^{ \pm}(0)=2.02 \pm 0.04
$$

Note that the form factors $F_{A}$ and $F_{E}$ both contain second-class current terms through the impulse-approximation coupling constant, $g_{\mathrm{T}}$. Thus, the analysis proceeds in one of two ways: either one accepts the CVC prediction, Eq.50, and uses additional data to set limits on second-class currents, or one sets $g_{\mathrm{T}}=0$ and uses additional data to verify the $\mathrm{CVC}$ hypothesis. Following the latter choice, and deducing axial form factors from the experimental half-lives 51 to be $F_{A}^{-}(0)=0.512 \pm 0.001$ and $F_{A}^{+}(0)=$ $0.481 \pm 0.002$, the slope parameters, $a_{ \pm}$, (see Eq.45) are predicted from CVC to be

$$
\begin{aligned}
a_{-} & =5.61+1.31=6.92 \mathrm{GeV}^{-1} \\
a_{+} & =-5.97+2.69=-3.28 \mathrm{GeV}^{-1} \\
\Delta a & =a_{-}-a_{+}=10.20 \pm 0.16 \mathrm{GeV}^{-1}
\end{aligned}
$$

The order- $\alpha Z$ corrections $44, \delta_{ \pm}(\alpha Z)$, which appear explicitly as the second terms in the equations for $a_{ \pm}$, are significant and shift the prediction away from the symmetric result of $a_{-} \simeq-a_{+}$expected in the absence of any isospin-symmetry breaking. Behrens and Syzbisz 4 also predict a sizeable quadratic term in the shape correction factor, $b_{ \pm} W^{2}$, in Eq. 44, which should be included in the analysis. However the contribution from this term is roughly the same in $e^{-}$and $e^{+}$transitions, so it can be omitted providing we restrict our attention to $\Delta a$, which is more reliable experimentally and theoretically since many systematic uncertainties cancel out.

The CVC hypothesis alone cannot predict values for the correlation coefficients, $\alpha_{ \pm}$, because of the presence of the $F_{E}$ form factor in Eq. 45 . However a prediction can be given for the difference 43 :

$$
\Delta \alpha=\alpha_{-}-\alpha_{+}=2.89 \pm 0.04 \mathrm{GeV}^{-1}
$$

where, as in Eq.51, the quoted error reflects only the uncertainty in the $M 1 \gamma$-decay width, $\Gamma_{\gamma}$. The experimental test of the CVC hypothesis, therefore, is to measure the difference in slope parameters, $\Delta a$, or the correlation coefficients, $\Delta \alpha$, in the mirror mass-12 transitions and compare with the predictions in Eqs.51 and 52. We consider some examples in the following sections.

\subsubsection{The total decay rate}

For non-aligned ${ }^{12} \mathrm{~B}$ and ${ }^{12} \mathrm{~N}$ nuclei, we can integrate over electron directions and energies in Eq.44, giving for the ratio of decay rates in the mirror transitions the result 


$$
\begin{aligned}
\frac{f t^{+}}{f t^{-}} & =\frac{\left|F_{A}^{-}(0)\right|^{2}}{\left|F_{A}^{+}(0)\right|^{2}} \frac{1+\eta_{-}+a_{-} W_{0}^{-} / 2}{1+\eta_{+}+a_{+} W_{0}^{+} / 2} \\
& \rightarrow 1-\frac{4}{3} \frac{W_{0}^{+}+W_{0}^{-}}{2 M} \frac{g_{\mathrm{T}}}{g_{\mathrm{A}}}+\delta_{\text {nucl }}
\end{aligned}
$$

where $f$ is the integrated Fermi function (see Eq. 32). The energy-dependent term, $a W$, has been replaced by its average value, $a W_{0} / 2$, and terms proportional to $\left(W_{0}^{+}-W_{0}^{-}\right)$ have been neglected. The second line represents the impulse-approximation limit. It would seem that a comparison of $f t$ values in mirror transitions would be a profitable way to search for second-class currents. This, indeed, was proposed by Wilkinson 52 and first results appeared encouraging. However, a definitive experiment was performed by Wilkinson and Alburger 53 in which the asymmetry of $f t$ values was measured as a function of $\left(W_{0}^{+}+W_{0}^{-}\right)$in the beta decays of ${ }^{8} \mathrm{Li}$ and ${ }^{8} \mathrm{~B}$. This is a unique case in which the daughter state is broad, decaying itself by $\alpha$ emission. This effectively permits the beta end-point energy to be varied within a single decay. Their results showed the ratio of $f t$ values to be almost independent of $\left(W_{0}^{+}+W_{0}^{-}\right)$, implying little or no evidence for second-class currents.

Another difficulty with this type of analysis concerns the existence of the additional term $\delta_{\text {nucl }}$ in Eq.53. It is given by $\left|\langle\boldsymbol{\sigma}\rangle^{-} /\langle\boldsymbol{\sigma}\rangle^{+}\right|^{2}=1+\delta_{\text {nucl }}$ and arises from the fact that $\langle\boldsymbol{\sigma}\rangle^{+} \neq\langle\boldsymbol{\sigma}\rangle^{-}$since the wavefunctions for ${ }^{12} \mathrm{~B}$ and ${ }^{12} \mathrm{~N}$ are not exact isospin eigenstates: the isospin symmetry is broken by the internucleon electromagnetic interaction and the neutron-proton mass difference. Nuclear-structure calculation\$54.55 indeed compute values for $\delta_{\text {nucl }}$ that are comparable with the total experimental asymmetry, and thus mask the evidence, if any, for the presence of second-class currents.

\subsubsection{Electron-spectrum shape}

To study the electron-energy spectrum for non-aligned ${ }^{12} \mathrm{~B}$ and ${ }^{12} \mathrm{~N}$ nuclei, we integrate only over electron directions in Eq. (44). A measurement of the spectrum makes it possible to determine the coefficient of the energy-dependent term $a_{ \pm} W$. This is the classic experiment of Lee et al. 5 , which first claimed to verify CVC. However, a reappraisal by Calaprice and Holstein 50 questioned the original analysis of the experiment and weakened the experimental support for the validity of CVC. The critique has been

discussed in a new analysis by the original group of experimenters5 5 using the data from the original experiment, with the conclusion that their data still support CVC. At the same time, a new measurement of $a_{ \pm}$was performed by Kaina et al. 8 using a NaI crystal as a spectrum analyzer instead of the magnetic spectrometer employed in the earlier measurements. The results for $a_{ \pm}$were not in agreement with previous (revised or not) determinations.

One cause of the trouble lies in the unresolved decay branch from ${ }^{12} \mathrm{~N}$ to the first excited state in ${ }^{12} \mathrm{C}$. The conclusion derived from the experiment depends strongly upon 
Table 3. Difference of slope parameters, $\Delta a$, for ${ }^{12} \mathrm{~B}$ and ${ }^{12} \mathrm{~N}$ mirror beta decays.

\begin{tabular}{|c|c|c|}
\hline $\begin{array}{c}\Delta a \\
\left(\mathrm{GeV}^{-1}\right)\end{array}$ & $\begin{array}{l}b_{1}^{+a} \\
(\%)\end{array}$ & Remarks \\
\hline $9.2 \pm 1.2$ & 2.4 & ref. 60 , as revised by 50 and $a_{+}$increased by 0.7 \\
\hline $15.3 \pm 2.8$ & 2.4 & ref.61, as revised by 50 and $a_{+}$increased by 0.7 \\
\hline $8.6 \pm 2.4$ & 2.1 & ref. 57 \\
\hline $9.1 \pm 0.9$ & 1.8 & ref. 58 , with $a_{+}$decreased by 0.7 \\
\hline $10.5 \pm 2.1$ & & ref.62, branching ratio, $b_{1}^{+}$, not given \\
\hline
\end{tabular}

${ }^{a}$ Branching ratio assumed in original reference.

the knowledge of the branching ratio, $b_{1}^{+}$, for the decay to that state, which must be subtracted from the observed result. For example 5 , a change from $1.8 \%$ to $2.1 \%$ in $b_{1}^{+}$ produces a variation in the extracted $a_{+}$of $-0.7 \mathrm{GeV}^{-1}$, a $25 \%$ change. A second cause of trouble lies in the systematic uncertainties surrounding the experimental energydependent response functions. So far, these seem insurmountable in the determination of $a_{ \pm}$, but are deemed largely to cancel in $\Delta a$; as a result, it is the latter quantity that we quote here. In Table 3 are the results from four experiments as corrected for a common $2.1 \%$ branching ratio as reviewed by Grenacs 59 , and a preliminary result 62 from a fifth experiment. The weighted average is $\Delta a=9.5 \pm 0.7$. By combining in quadrature the effects of an uncertainty of $0.3 \%$ in $b_{1}^{+}$, we produce a recommended value of

$$
\Delta a=9.5 \pm 1.0 \mathrm{GeV}^{-1},
$$

which agrees with the CVC prediction given in Eq.51.

\subsubsection{Polarization and alignment measurements}

We now turn to experiments with oriented nuclei and measurements of the decay electron's angular distribution as given in Eq. 44:

$$
W\left(\theta_{e}\right)=1 \pm\left(h_{1}-h_{-1}\right)\left(1+\alpha_{ \pm} W\right) P_{1}\left(\cos \theta_{e}\right)+\left(1-3 h_{0}\right) \alpha_{ \pm} W P_{2}\left(\cos \theta_{e}\right) .
$$

Typical experimental arrangements are described by Minamisono63, Haskell and Madansky 64 and Haskell et al.65. Polarized ${ }^{12} \mathrm{~B}$ and ${ }^{12} \mathrm{~N}$ recoils are produced through the reactions ${ }^{10} \mathrm{~B}\left({ }^{3} \mathrm{He}, n\right){ }^{12} \mathrm{~N}$ and ${ }^{11} \mathrm{~B}(d, p){ }^{12} \mathrm{~B}$, with the precise magnetic substate populations of the product nuclei depending on the incident beam energy and the recoil angle. The polarized recoiling nuclei are implanted into monocrystalline metal foils in 
Table 4. Beta-decay asymmetry coefficients, $\alpha_{ \pm}$in $\mathrm{GeV}^{-1}$.

\begin{tabular}{|c|c|c|}
\hline$\alpha_{-}\left({ }^{12} \mathrm{~B}\right)$ & $\alpha_{+}\left({ }^{12} \mathrm{~N}\right)$ & Reference \\
\hline$-(0.07 \pm 0.20)$ & & Lebrun et al. 97 \\
\hline$+(0.24 \pm 0.44)$ & & Brändle et al.68 \\
\hline$+(0.25 \pm 0.34)$ & $-(2.77 \pm 0.52)$ & Sugimoto et al.69 \\
\hline$+(0.10 \pm 0.30)$ & $-(2.73 \pm 0.39)$ & Brändle et al. 70 \\
\hline$-(0.01 \pm 0.21)$ & $-(2.67 \pm 0.56)$ & Masuda et al. 7 \\
\hline$+(0.046 \pm 0.053)$ & $-(2.795 \pm 0.100)$ & Minamisono et al. 72 \\
\hline$-(0.174 \pm 0.059)$ & $-(2.774 \pm 0.086)$ & Minamisono et al.73 \\
\hline$-(0.043 \pm 0.046)$ & $-(2.780 \pm 0.063)$ & Average values \\
\hline
\end{tabular}

a large magnetic holding field in which the degeneracy of the Zeeman levels is broken by the quadrupole couplings. The polarization is detected through an asymmetry in the angular distribution of the electrons in the subsequent $\beta$ decays.

The first determinations 66 of $\alpha_{ \pm}$from the $\beta$-energy dependence of the $P_{1}\left(\cos \theta_{e}\right)$ term in the decay electron's angular distribution (see Eq. 55) turned out to be fraught with experimental difficulties (primarily from backscattering), since a small effect is to be isolated from a large leading term (i.e. $\alpha W \ll 1$ ). More reliable data have come from later experiments that concentrated on the $P_{2}\left(\cos \theta_{e}\right)$ term in Eq. 55. A summary of experimental results is given in Table $甘$. The data are all in accord and the average value for the difference in the mirror correlation coefficients is

$$
\Delta \alpha=\alpha_{-}-\alpha_{+}=2.737 \pm 0.078 \mathrm{GeV}^{-1}
$$

This result is in good agreement with the CVC expectation given in Eq. 52 .

\subsection{4. $\beta-\alpha$ correlations}

A similar test of CVC can be mounted in the mass- 8 mirror transitions ${ }^{8} \mathrm{Li}\left(2^{+}, 1\right) \rightarrow$ ${ }^{8} \mathrm{Be}\left(2^{+}, 0\right)+e^{-}+\bar{\nu}_{e}$ and ${ }^{8} \mathrm{~B}\left(2^{+}, 1\right) \rightarrow{ }^{8} \mathrm{Be}\left(2^{+}, 0\right)+e^{+}+\nu_{e}$. The broad daughter state, which becomes aligned with respect to the electron direction in the process, subsequently breaks up into a pair of $\alpha$ particles. The degree of alignment is measured from the $\cos ^{2} \theta$ distribution of $\alpha$-particles, where $\theta$ is the angle between the electron and $\alpha$-particle directions. The $\beta$ - $\alpha$ directional correlation in the $\mathrm{A}=8$ nuclei and the electron angular distribution from aligned $A=12$ nuclei are therefore essentially equivalent phenomena 59 with the role of the initial and final states inverted.

The CVC prediction for this case requires the analogous M1 $\gamma$-transition to be measured, but this poses more problems than in the mass-12 case. Firstly, the $2^{+} \rightarrow 2^{+}$ 
sequence implies that the electromagnetic transition is a mix of M1 and E2 multipoles, so a mixing ratio has to be measured to determine the M1 fraction. Secondly, the analogue state of the decay parent in ${ }^{8} \mathrm{Be}$ is a nearly degenerate doublet (16.6 and 16.9 $\mathrm{MeV}$ ) of which both members are roughly equal mixes of $\mathrm{T}=0$ and $\mathrm{T}=1$ eigenstates. De Braeckeleer 74 recently reported on a determination of the M1 and E2 widths needed for the CVC test in which the ${ }^{4} \mathrm{He}(\alpha, \gamma)$ cross section was measured as a function of energy across the 16.6 and $16.9 \mathrm{MeV}$ levels and photon angular distributions were obtained for both resonances. The analysis leads to an isovector $\mathrm{M} 1$ width of $2.80 \pm 0.18 \mathrm{eV}$, in serious disagreement with previous determinations by Bowles and Garvey 70 . 7 .

The $\beta-\alpha$ correlations in the two mirror decays have been measured by Tribble and Garvey 77 and the results are in agreement with the CVC prediction based on the earlier M1 width, although there are large uncertainties. A subsequent experiment 8 detecting both alpha particles enabled the correlation to be determined as a function of the final-state energy. The results show a deviation from the CVC prediction, which is dependent on the final-state energy. A repeat experiment 49 is currently underway, in which it is proposed to measure the triple $\beta-\nu-\alpha$ angular correlation. The neutrino direction will be inferred from the recoil of the second $\alpha$ particle. Furthermore, by an appropriate choice of geometry, it may be possible to separate the weak-magnetism and the second-class-current contributions to the alignment. The experiment 49 is expected to improve present limits on second-class currents by a factor of five.

\subsection{5. $\beta-\gamma$ correlations}

Another similar test of CVC is based on the mass-20 mirror transitions ${ }^{20} \mathrm{~F}\left(2^{+}, 1\right)$ $\rightarrow{ }^{20} \mathrm{Ne}\left(2^{+}, 0\right)+e^{-}+\bar{\nu}_{e}$ and ${ }^{20} \mathrm{Na}\left(2^{+}, 1\right) \rightarrow{ }^{20} \mathrm{Ne}\left(2^{+}, 0\right)+e^{+}+\nu_{e}$. In this case, the daughter nucleus de-excites by $\gamma$-ray emission rather than $\alpha$ emission as in the mass- 8 case. The $\beta-\gamma$ angular correlation measurement is equivalent to the $\beta-\alpha$ correlation experiment and the analysis therefore follows analogously. The result, $89,80,81$ confirm the CVC hypothesis, but only to an accuracy of $\pm 25 \%$.

\section{Axial-vector Interaction in the Nucleon}

\subsection{Partially Conserved Axial-vector Current Hypothesis}

We start with a small aside and consider the decay of the pion: $\pi^{-} \rightarrow \ell^{-} \bar{\nu}_{\ell}$, where $\ell$ represents either the first generation, $e^{-} \bar{\nu}_{e}$, or second generation, $\mu^{-} \bar{\nu}_{\mu}$, leptons. Since the pion is a bound state of $d$ and $\bar{u}$ quarks, its decay is a straightforward example of a semi-leptonic charged-current weak interaction. The $T$-matrix for the decay process is given by Eq. 4 , and is easily computed for free quarks. However, for pion decay, the quarks are bound within the pion and, as mentioned in sect.1.3, the required quark density functions are not reliably computed. Thus, a more phenomenological approach is followed in which we parameterize the matrix element of the quark hadronic

current, $J_{\mu}^{h}$, evaluated between the pion and the vacuum, $\left\langle 0\left|J_{\mu}^{h}\right| \pi^{-}\right\rangle$. Since the pion is 
spinless, the only available four-vector is the momentum transfer between the hadron and leptons, $k_{\mu}$. Thus, the current is parameterized as

$$
J_{\mu}^{h}=i f_{\pi} k_{\mu} \pi^{a},
$$

where $\pi^{a}$ is the field operator for the pion, with the superscript $a$ an isospin index, and $f_{\pi}$ is some real numerical constant, which parameterizes our inability to evaluate the hadron matrix element. In principle, $f_{\pi}$ should be calculable in QCD. Note that since the pion is a pseudoscalar particle and $k_{\mu}$ a polar vector, only the axial portion of $J_{\mu}^{h}$, namely $A_{\mu}^{h}$, is operative in Eq.57. The lifetime for the decay is evaluated in a standard way 82 to give:

$$
\tau^{-1}\left(\pi^{-} \rightarrow \ell^{-} \bar{\nu}_{\ell}\right)=\frac{G_{\mathrm{F}}^{2} f_{\pi}^{2}}{4 \pi} V_{u d}^{2} m_{\ell}^{2} m_{\pi}\left(1-\frac{m_{\ell}^{2}}{m_{\pi}^{2}}\right)^{2} .
$$

The decay is predominately to muons. Thus, with the measured pion lifetime, corrected for other small branches and adjusted by a few percent for radiative corrections, a value for $f_{\pi}$ is determined from Eq. 58 with the known values of $m_{\mu}, m_{\pi}, G_{\mathrm{F}}$ and $V_{u d}$. The result is $\#$

$$
f_{\pi}=0.663 m_{\pi}=92.42 \pm 0.26 \mathrm{MeV} .
$$

The constant, $f_{\pi}$, is called the pion-decay constant.

The axial-vector current has no electromagnetic analogue. Furthermore, it is not a conserved current since the pion does decay. The axial current and its derivative for pion decay are therefore

$$
\begin{aligned}
A_{\mu}^{h} & =i f_{\pi} k_{\mu} \pi^{a} \\
\partial_{\mu} A_{\mu}^{h} & =i k_{\mu} A_{\mu}^{h}=-f_{\pi} k_{\mu}^{2} \pi^{a}=m_{\pi}^{2} f_{\pi} \pi^{a},
\end{aligned}
$$

where $k_{\mu}^{2}=-m_{\pi}^{2}$ for free pions. Its divergence is non-zero because $f_{\pi} \neq 0$ and $m_{\pi} \neq 0$. However the pion is light on the hadronic mass scale and its mass can be considered small. Gell-Mann and Levy 84 introduced the concept of a partially conserved axial current (PCAC) by postulating that conservation is achieved in the "soft-pion limit", in which the pion mass tends to zero. Thus, the divergence of the axial current appears to be related to the creation operator for a pion. If this principle is also applied to the axial current operative in the decay of the neutron, it can be assumed that the current is dominated by the pion-pole graph. That is, a pion is created at the proton-neutron vertex and then subsequently decays. Schematically, the axial current is 82

\#4 Note our definition of $f_{\pi}$ differs from the Particle Data group 6 by $\sqrt{2}$ 


$$
\begin{aligned}
A_{\mu}^{h} & =\left(n \rightarrow p \pi^{-} \text {vertex }\right) \times\left(\pi^{-} \text {propagator }\right) \times\left(\pi^{-} \rightarrow \mathrm{e}^{-} \bar{\nu}_{\mathrm{e}} \text { vertex }\right) \\
& =i g_{\pi \mathrm{NN}} F_{\pi \mathrm{NN}}\left(k^{2}\right) \bar{\psi} \gamma_{5} \psi \tau^{a} \times\left(k^{2}+m_{\pi}^{2}\right)^{-1} \times i f_{\pi} k_{\mu} \\
& =i \frac{2 g_{\pi \mathrm{NN}} F_{\pi \mathrm{NN}}\left(k^{2}\right) f_{\pi}}{k^{2}+m_{\pi}^{2}} \bar{\psi}\left[i k_{\mu} \gamma_{5}\right] \psi \frac{1}{2} \tau^{a}
\end{aligned}
$$

where $g_{\pi \mathrm{NN}}$ is the pion-nucleon coupling constant. Here $F_{\pi \mathrm{NN}}\left(k^{2}\right)$ is a vertex form factor, taken to be a smooth function of $k^{2}$ with $F_{\pi \mathrm{NN}}\left(k^{2}=-m_{\pi}^{2}\right)=1$ so that $g_{\pi \mathrm{NN}}$ becomes the physically-measured coupling constant. Thus, the derivative of this current in the soft-pion limit (neglecting the derivative of the vertex form factor) is

$$
\lim _{m_{\pi}^{2} \rightarrow 0} \partial_{\mu} A_{\mu}^{h}=-i 2 g_{\pi \mathrm{NN}} F_{\pi \mathrm{NN}}\left(k^{2}\right) f_{\pi} \bar{\psi}\left[\gamma_{5}\right] \psi \frac{1}{2} \tau^{a} .
$$

Another possible pole graph involves the $A_{1}$-meson, a meson of the same spinparity quantum numbers as the axial current. Then the axial current is

$$
\begin{aligned}
A_{\mu}^{h} & =\left(n \rightarrow p A_{1} \text { vertex }\right) \times\left(\mathrm{A}_{1} \text { propagator }\right) \times\left(\mathrm{A}_{1} \rightarrow \mathrm{e}^{-} \bar{\nu}_{\mathrm{e}} \text { vertex }\right) \\
& =\left(-i g_{\mathrm{A}} g_{\rho} F_{\mathrm{ANN}}\left(k^{2}\right) \bar{\psi} \gamma_{\mu} \gamma_{5} \tau^{a} \psi\right) \times\left(\frac{\delta_{\mu \nu}+k_{\mu} k_{\nu} / m_{\mathrm{A}}^{2}}{k^{2}+m_{\mathrm{A}}^{2}}\right) \times\left(-\frac{m_{\mathrm{A}}^{2}}{2 g_{\rho}}\right) \\
& =i g_{\mathrm{A}} F_{\mathrm{ANN}}\left(k^{2}\right) \frac{m_{\mathrm{A}}^{2}}{k^{2}+m_{\mathrm{A}}^{2}} \bar{\psi}\left[\gamma_{\mu} \gamma_{5}+\frac{k_{\mu} \not k \gamma_{5}}{m_{\mathrm{A}}^{2}}\right] \psi \frac{1}{2} \tau^{a},
\end{aligned}
$$

where $\not k=k_{\alpha} \gamma_{\alpha}$. The $A_{1} N N$ coupling constant is extracted from a phenomenological chirally-invariant Lagrangian 85 in which the $A_{1}$-meson is treated as the chiral partner of the $\rho$-meson, and its mass related to that of the $\rho$-meson via the Weinberg relation 86 : $m_{\mathrm{A}}=\sqrt{2} m_{\rho}$. In particular, we quote from the work of Ivanov and Truhlik 6 where $g_{A_{1} N N}=g_{\mathrm{A}} g_{\rho}$ with $g_{\rho}$ the coupling constant for the decay of the rho-meson to two pions. Similarly, in the same Lagrangian, the decay of the $A_{1}$-meson to leptons is given in terms of the same coupling constant, $g_{\rho}$, which then cancels out in forming the axial current in Eq. 63. This is the feature of chiral Lagrangians that all vertices involving $\rho$ and $A_{1}$ mesons are expressed in terms of a common coupling constant, here $g_{\rho}$. Again, a smooth vertex function has been introduced with $F_{\text {ANN }}\left(k^{2}=-m_{\mathrm{A}}^{2}\right)=1$. The derivative of this current is

$$
\begin{aligned}
\partial_{\mu} A_{\mu}^{h} & =-g_{\mathrm{A}} F_{\mathrm{ANN}}\left(k^{2}\right) \frac{m_{\mathrm{A}}^{2}}{k^{2}+m_{\mathrm{A}}^{2}} \bar{\psi}\left[\not k \gamma_{5}+\frac{k_{\mu} \not k \gamma_{5}}{m_{\mathrm{A}}^{2}}\right] \psi \frac{1}{2} \tau^{a} \\
& =-g_{\mathrm{A}} F_{\mathrm{ANN}}\left(k^{2}\right) \bar{\psi}\left[\not k \gamma_{5}\right] \psi \frac{1}{2} \tau^{a} \\
& =2 i g_{\mathrm{A}} F_{\mathrm{ANN}}\left(k^{2}\right) M \bar{\psi}\left[\gamma_{5}\right] \psi \frac{1}{2} \tau^{a}
\end{aligned}
$$


with $M$ the nucleon mass. Then, if the pion- and $A_{1}$-meson pole graphs, Eqs. 62 and 64 , are added together, the derivative of the axial current in the soft-pion limit becomes

$$
\lim _{m_{\pi}^{2} \rightarrow 0} \partial_{\mu} A_{\mu}^{h}=2 i\left(g_{\mathrm{A}} F_{\mathrm{ANN}}\left(k^{2}\right) M-g_{\pi \mathrm{NN}} F_{\pi \mathrm{NN}}\left(k^{2}\right) f_{\pi}\right) \bar{\psi}\left[\gamma_{5}\right] \psi \frac{1}{2} \tau^{a},
$$

and the requirement that this derivative vanish leads to the condition

$$
g_{\mathrm{A}} F_{\mathrm{ANN}}\left(k^{2}\right) M=g_{\pi \mathrm{NN}} F_{\pi \mathrm{NN}}\left(k^{2}\right) f_{\pi} .
$$

If, in addition, it is assumed that the vertex form factors are slowly varying functions of $k^{2}$, such that they can be replaced by their limiting values of unity, then

$$
g_{\mathrm{A}} M=g_{\pi \mathrm{NN}} f_{\pi} .
$$

This result is known as the Goldberger-Treiman relation.

The accepted value for $g_{\pi \mathrm{NN}}$ for many years was that determined from $\pi$-nucleon scattering data by Bugg, Carter and Carter 8 of $13.40 \pm 0.08$ and confirmed by the Karlsruhe-Helsinki 89 group. Recently, this value has been challenged, notably by the Nijmegen group 90 , and smaller values are currently recommended. Three recent determinations are: $13.03 \pm 0.03$ (ref.90), $13.14 \pm 0.07$ (ref.91), and $13.12 \pm 0.13$ (ref.92). Since this issue is still under discussion we will use the last value as a conservative estimate for what follows. With this choice for $g_{\pi \mathrm{NN}}$, the pion-decay constant from Eq. 59, and the average of the proton and neutron masses, $M=938.919 \mathrm{MeV}$, the Goldberger-Treiman relation predicts for the axial-vector constant $\left|g_{\mathrm{A}}\right|=1.29 \pm 0.01$. This is reasonably close to the value 1.26 obtained from neutron beta decay (see next section). The difference of only $2 \%$ is remarkable testimony to the accuracy of the PCAC hypothesis.

Finally, the sum of Eqs.61 and 63 yield for the total axial current

$$
\begin{aligned}
A_{\mu}^{h} & =i g_{\mathrm{A}} F_{\mathrm{ANN}}\left(k^{2}\right) \bar{\psi}\left[\frac{m_{\mathrm{A}}^{2}}{k^{2}+m_{\mathrm{A}}^{2}} \gamma_{\mu} \gamma_{5}-\frac{\left(m_{\mathrm{A}}^{2}-m_{\pi}^{2}\right)}{\left(k^{2}+m_{\mathrm{A}}^{2}\right)\left(k^{2}+m_{\pi}^{2}\right)} k_{\mu} \not k \gamma_{5}\right] \psi \frac{1}{2} \tau^{a} \\
& =i g_{\mathrm{A}} F_{\mathrm{ANN}}\left(k^{2}\right) \frac{m_{\mathrm{A}}^{2}}{k^{2}+m_{\mathrm{A}}^{2}} \bar{\psi}\left[\gamma_{\mu} \gamma_{5}+\frac{2 M i\left(1-m_{\pi}^{2} / m_{\mathrm{A}}^{2}\right)}{k^{2}+m_{\pi}^{2}} k_{\mu} \gamma_{5}\right] \psi \frac{1}{2} \tau^{a},
\end{aligned}
$$

which can be compared with the phenomenological expression of Eq.12 to obtain expressions for the form factors $g_{\mathrm{A}}\left(k^{2}\right)$ and $g_{\mathrm{P}}\left(k^{2}\right)$. The results are

$$
g_{\mathrm{A}}\left(k^{2}\right)=g_{\mathrm{A}} F_{\mathrm{ANN}}\left(k^{2}\right) \frac{m_{\mathrm{A}}^{2}}{k^{2}+m_{\mathrm{A}}^{2}}
$$

and

$$
g_{\mathrm{P}}\left(k^{2}\right)=\frac{2 M g_{\mathrm{A}}\left(k^{2}\right)}{k^{2}+m_{\pi}^{2}}\left(1-\frac{m_{\pi}^{2}}{m_{\mathrm{A}}^{2}}\right),
$$

or 


$$
g_{\mathrm{P}}\left(k^{2}\right)=\frac{2 g_{\pi \mathrm{NN}} f_{\pi}}{k^{2}+m_{\pi}^{2}} F_{\pi \mathrm{NN}}\left(k^{2}\right) .
$$

Since these derivations are based on meson-pole graphs, the form factors deduced are of monopole form, with the range given by the mass of the appropriate meson. For $g_{\mathrm{A}}\left(k^{2}\right)$, use of a phenomenological chiral Lagrangian has fixed $m_{\mathrm{A}}=\sqrt{2} m_{\rho}=$ $1086 \mathrm{MeV}$, a value not too far from the physical $A_{1}$ meson mass 6 of $1230 \pm 40 \mathrm{MeV}$. Experimental information has also been obtained from neutrino-nucleon scattering 93 at the Brookhaven AGS, where the antineutrino quasielastic reaction $\bar{\nu}_{\mu} p \rightarrow \mu^{+} n$ was studied in the momentum range up to $k^{2} \sim 1.0 \mathrm{GeV}^{2}$. A value of an axial-vector mass, $m_{\mathrm{D}}$, in a dipole parameterization of the form factor was determined from the shape of the momentum distribution to be $1.09 \pm 0.04 \mathrm{GeV}$. This agrees with the monopole value, $m_{\mathrm{A}}$, obtained from the phenomenological chiral Lagrangian and suggests the vertex form factor $F_{\mathrm{ANN}}\left(k^{2}\right)$ is likewise a monopole function of similar range.

In Eqs.70 and 71 we gave two expressions for the pseudoscalar coupling constant. The first uses the Goldberger-Treiman relation and includes a factor $\left(1-m_{\pi}^{2} / m_{\mathrm{A}}^{2}\right)$, which originates in the propagator for the $A_{1}$-meson. The second expression assumes that the pseudoscalar coupling constant is entirely due to the pion-pole graph, and is the expression we will use here. In semi-leptonic decays, the pseudoscalar coupling constant is multiplied by the mass of the lepton, $m_{\ell}$, in the expression for the transition rate. Thus, for processes involving first generation leptons, such as nuclear beta decay, the pseudoscalar term gives a negligible contribution: see sect.3.3. In contrast, for processes such as muon capture, involving second-generation leptons, the contribution is significant and characterized by the dimensionless quantity

$$
m_{\mu} \frac{g_{\mathrm{P}}\left(k^{2}\right)}{g_{\mathrm{A}}(0)}=\frac{2 g_{\pi \mathrm{NN}} f_{\pi} m_{\mu}}{g_{\mathrm{A}}\left(k^{2}+m_{\pi}^{2}\right)} F_{\pi \mathrm{NN}}\left(k^{2}\right)=7.04 \pm 0.07,
$$

in which $g_{\pi_{\mathrm{NN}}}=13.12 \pm 0.13, f_{\pi}=92.42 \pm 0.26 \mathrm{MeV}, g_{\mathrm{A}}=1.2599 \pm 0.0025$ from neutron decay and $F_{\pi \mathrm{NN}}\left(k^{2}\right) \simeq 1$. A correction to this expression has recently been given by Bernard, Kaiser and Meissner 94. It is based on the chiral Ward identity of QCD and exploits heavy-meson chiral perturbation theory to find an expression to order $k^{2}$. The result is

$$
m_{\mu} \frac{g_{\mathrm{P}}\left(k^{2}\right)}{g_{\mathrm{A}}(0)}=\frac{2 g_{\pi \mathrm{NN}} f_{\pi} m_{\mu}}{g_{\mathrm{A}}\left(k^{2}+m_{\pi}^{2}\right)}-\frac{1}{3} m_{\mu} M r_{\mathrm{A}}^{2},
$$

where $r_{\mathrm{A}}^{2}$ is the mean square axial radius of the nucleon obtained from the axial-vector form factor: $g_{\mathrm{A}}\left(k^{2}\right)=g_{\mathrm{A}}(0)\left(1-\frac{1}{6} k^{2} r_{\mathrm{A}}^{2}\right)$. In dipole fits $93, r_{\mathrm{A}}=\sqrt{12} / m_{\mathrm{D}}=0.63 \pm$ $0.02 \mathrm{fm}$. corresponding to $m_{\mathrm{D}}=1.09 \pm 0.04 \mathrm{GeV}$. The prediction for the dimensionless pseudoscalar quantity then becomes

$$
m_{\mu} \frac{g_{\mathrm{P}}\left(k^{2}\right)}{g_{\mathrm{A}}(0)}=(7.04 \pm 0.07)-(0.33 \pm 0.02)=6.71 \pm 0.08
$$


In both Eqs.72 and 74, the numerical result is obtained for a value of $k^{2}$ appropriate for muon capture $\left(k^{2}=-m_{\mu}^{2}+2 m_{\mu} Q \simeq 0.88 m_{\mu}^{2}\right)$ where $Q$ is the reaction Q-value. A measurement of $g_{\mathrm{P}}$ in muon capture by hydrogen, in addition to providing a test of PCAC and the dominance of the pion pole, could, if sufficient accuracy were achieved, confirm or deny the correction term predicted 3 from heavy-meson chiral perturbation theory.

\subsection{Neutron Decay}

The decay of the neutron is the simplest $\beta$-decay mode that involves both the vector and the axial-vector interactions. In principle, its study should provide us with the most direct view of $g_{\mathrm{A}}$ and $g_{\mathrm{v}}$, uncluttered by complex nuclear wave functions or by renormalization effects of the nuclear medium. In practice, however, the experimental uncertainties have, until recently, been large. The value of $g_{\mathrm{v}}$, as determined from neutron decay, is a factor of five less precise than the value obtained from the $0^{+} \rightarrow 0^{+}$ nuclear $\beta$-decays (see sect.2.2). Ultimately, though, its potential is greater since the nuclear result has reached a plateau established by theoretical uncertainties that are not likely to be soon reduced.

In the same notation used to describe the superallowed nuclear decays (see Eq. 27), the neutron decay rate can be written:

$$
f t\left(1+\delta_{R}\right)=\frac{K}{\left(G_{\mathrm{V}}^{\prime 2}\left\langle M_{\mathrm{V}}\right\rangle^{2}+G_{\mathrm{A}}^{\prime 2}\left\langle M_{\mathrm{A}}\right\rangle^{2}\right)},
$$

with

$$
\begin{gathered}
G_{\mathrm{V}}^{\prime 2}=\left(1+\Delta_{\mathrm{R}}^{\mathrm{v}}\right) G_{\mathrm{V}}^{2} \\
G_{\mathrm{A}}^{\prime 2}=\left(1+\Delta_{\mathrm{R}}^{\mathrm{A}}\right) G_{\mathrm{A}}^{2} .
\end{gathered}
$$

Here $G_{\mathrm{V}}=G_{\mathrm{F}} V_{u d}$ and $G_{\mathrm{A}}=G_{\mathrm{F}} V_{u d} g_{\mathrm{A}}$ with $G_{\mathrm{F}}$ the fundamental weak-interaction coupling constant determined from muon decay, to which all other coupling constants are referenced. For neutron decay, $\left\langle M_{\mathrm{V}}\right\rangle^{2}=1$ and $\left\langle M_{\mathrm{A}}\right\rangle^{2}=3$.

Although the calculation of the matrix elements, $M_{\mathrm{V}}$ and $M_{\mathrm{A}}$, does not offer any theoretical challenge in the case of neutron decay, it is evident that a measurement of the neutron's $f t$-value must be complemented by one additional measurement before useful information can be extracted on the individual coupling constants, $G_{\mathrm{V}}^{\prime}$ and $G_{\mathrm{A}}^{\prime}$. To appreciate what other measurements are possible, we write the differential of the probability distribution for neutron $\beta$ decay, $n \rightarrow p e^{-} \bar{\nu}_{e}$, in the following form 9 :

$$
\mathrm{d}^{5} \Gamma \propto N(W)\left[1+a \frac{v}{c} \hat{\mathbf{p}}_{e} \cdot \hat{\mathbf{p}}_{\bar{\nu}}+\left\langle\boldsymbol{\sigma}_{n}\right\rangle \cdot\left(A \frac{v}{c} \hat{\mathbf{p}}_{e}+B \hat{\mathbf{p}}_{\bar{\nu}}\right)\right] \mathrm{d} \Omega_{e} \mathrm{~d} \Omega_{\bar{\nu}} \mathrm{d} W,
$$

in which $N(W)$ is the usual allowed electron energy spectrum and $\left\langle\boldsymbol{\sigma}_{n}\right\rangle$ is the average neutron polarization vector. The three coefficients, $a, A$ and $B$, known respec- 
tively as the $e-\bar{\nu}$ angular-correlation coefficient, the $\beta$-asymmetry parameter and the $\bar{\nu}$-asymmetry parameter, are each related to the ratio of coupling constants: viz. ,

$$
a=\frac{1-\lambda^{2}}{1+3 \lambda^{2}} ; \quad A=-2 \frac{\lambda(\lambda+1)}{1+3 \lambda^{2}} ; \quad B=2 \frac{\lambda(\lambda-1)}{1+3 \lambda^{2}}
$$

with

$$
\lambda=G_{\mathrm{A}}^{\prime} / G_{\mathrm{V}}^{\prime} \simeq g_{\mathrm{A}} .
$$

All three coefficients have been measured and the first two, being more sensitive to $\lambda$, have been combined with the neutron's $f t$ value to determine $G_{\mathrm{V}}^{\prime}$ and $G_{\mathrm{A}}^{\prime}$.

\subsection{1. $f t$-value measurements}

As with nuclear decay, the $f t$ value for neutron decay depends upon its $Q_{E C}$ value half-life and branching ratio. The $Q_{E C}$ value is derived from the evaluated masses 96 of the neutron and proton, which are known to high precision via a combination of Penning-trap mass measurements (see, for example, ref. 97) and measurements of the neutron binding energy in deuterium (e.g. ref. 98). The result is $Q_{E C}=1293.3392(22)$ $\mathrm{keV}$.

Measurement of the neutron lifetime has defied such high levels of precision. Its relatively long, 10.2 min half-life, together with the difficulty in containing a welldefined source, have conspired to restrict precision to a half percent or so - more than an order-of-magnitude worse than the best nuclear-lifetime measurements - in spite of heroic efforts. The measurements (for a recent review, see ref.99) can be separated into two distinct categories: those in which the decay products are counted from a defined segment of a slow-neutron beam, and those in which cold neutrons are stored and their number measured after a well-determined time. These categories have been described 95 as "counting the dead" and "counting the survivors", respectively.

3.2.1.1 Neutron-beam experiments. These experiments depend upon an accurate knowledge of the efficiency, $\epsilon_{d}$, with which decay products can be detected, and upon the total number of neutrons, $N$, being viewed by the decay-product detectors. If $C_{d}$ is the observed decay rate, then

$$
C_{d}=\frac{N \epsilon_{d}}{\tau}=\frac{\rho_{N} V_{d} \epsilon_{d}}{\tau}
$$

Here $\tau$ is the neutron mean-life (conventionally used in this field instead of the halflife: $t=\tau \ln 2), \rho_{N}$ is the neutron density and $V_{d}$ is the sensitive decay volume. If a continuous neutron beam is used, special attention is required to define the boundaries of $V_{d}$; with a pulsed beam, $V_{d}$ is defined by the dimensions of the neutron packet, but at the expense of a reduced counting rate.

Results for the five most-precise beam measurements appear in Table 5 . Of the three continuous-beam experiments, one 100 detected the decay electrons, the other 
Table 5. Experimental results ${ }^{a}$ for neutron decay

\begin{tabular}{|c|c|c|c|c|c|}
\hline & \multirow[t]{2}{*}{ Method } & \multicolumn{2}{|c|}{ Measured values } & \multicolumn{2}{|c|}{ Average values } \\
\hline & & 1 & 2 & subsets & overall \\
\hline \multirow[t]{6}{*}{$\tau(\mathrm{s})$} & $n$ beam & $918 \pm 14100$ & $891 \pm 9$ & & \\
\hline & & $876 \pm 21$ & $878 \pm 31103$ & & \\
\hline & & $893.6 \pm 5.5204$ & & $894.2 \pm 4.7$ & \\
\hline & $n$ trap & $903 \pm 13105,106$ & $893 \pm 20106$ & & \\
\hline & & $877 \pm 10107$ & $887.6 \pm 3.0108$ & & \\
\hline & & $888.4 \pm 3.3109$ & $882.6 \pm 2.7 \pm 10$ & $885.9 \pm 1.7$ & $887.0 \pm 2.0$ \\
\hline \multirow[t]{4}{*}{$\lambda$} & $\beta$-asym. & $-1.254 \pm 0.015$ & $-1.257 \pm 0.012$ & & \\
\hline & & $\begin{array}{l}-1.262 \pm 0.005 \\
-1.266 \pm 0.004\end{array}$ & $-1.2544 \pm 0.0036 \underline{15}$ & $-1.2599 \pm 0.0026$ & \\
\hline & $e-\bar{\nu}$ corr. & $-1.259 \pm 0.017117$ & & $-1.259 \pm 0.017$ & \\
\hline & & & & & $-1.2599 \pm 0.0025$ \\
\hline
\end{tabular}

${ }^{a}$ Following the practice used for superallowed decay, we retain only those measurements with uncertainties that are within a factor of ten of the most precise measurement for each quantity. All such measurements not withdrawn by their authors, of which we are aware in early 1995 , are listed.

${ }^{b}$ Corrected for weak magnetism and recoil following ref. 112

two 101,104 detected protons. The most recent, by Byrne et al.104, stored the decay protons in a variable-length Penning trap, the magnetic axis of which coincided with the neutron-beam axis. To suppress background, the protons were released periodically in a brief burst and accelerated into a solid-state detector. The two remaining beam experiments used pulsed cold-neutron beams: the first 102 observed decay electrons with an in-line superconducting spectrometer, PERKEO; the other 103 used a helium-filled timeprojection chamber to observe the decay electrons and measure the neutron density in the same apparatus.

3.2.1.2 Stored-neutron experiments. Neutrons of sufficiently low energy $\left(\leq 10^{-7}\right.$ $\mathrm{eV}$ ) can be successfully confined by material walls, from which they are coherently scattered, by gravity or by a magnetic-field gradient acting on their magnetic moment. In experiments of this type, a reproducible but not necessarily well known number of neutrons is stored and then the number that remains after a storage time, $t$, is counted. The result is related to the neutron mean-life by

$$
N(t)=N(0) \exp \left[-t\left(\frac{1}{\tau}+\frac{1}{\tau_{\text {loss }}}\right)\right]
$$

in which $\tau_{\text {loss }}$ reflects the various neutron-loss processes except $\beta$ decay. The measurement is then repeated for many different storage times and under different conditions 
that affect the loss processes. Because the loss effects are deemed to be reasonably well understood, these results can be extrapolated to give a zero-loss limit, thus yielding the neutron mean-life.

Results for six measurements with neutron traps are listed in Table 5: five of the experiments used ultra-cold neutrons in gravitational traps with diverse wall materials; one employed a magnetic storage ring. The earliest trap 105 had aluminum walls, adjustable in temperature from 80 to $750 \mathrm{~K}$, and movable plates to vary the neutron collision frequency. This same apparatus was later used 106 with a thin layer of heavywater ice on the walls to reduce losses substantially. A wall coating of hydrogen-free diffusion-pump oil was used by Mampe et al. 108 to minimize loss in their variablevolume chamber, while Nesvizhevskii et al. 109 chose frozen oxygen on cooled beryllium walls. The most recent refinement 1110 combined oil-coated walls with on-line detection of neutrons inelastically scattered by the vessel wall, one of the most important contributions to neutron loss. Even the magnetic storage ring of Paul et al.107 suffered losses because some injected neutrons were captured in unstable orbits; it is believed that those neutrons disappeared rapidly and that decay at later times was entirely due to $\beta$ decay.

The lifetime measurements within each category are internally consistent but when they are considered as a single body of data the agreement is less satisfactory though acceptable: the confidence level is $\sim 25 \%$. It has been suggested 118 that the difference between the average lifetimes for beam and trap measurements could reflect the decay $n \rightarrow H \bar{\nu}_{e}$, which would be unobserved in the former. If one takes this approach, the averages quoted in Table 5 yield a branching-ratio limit for the hydrogenatom branch of $\leq 2 \%$ at the $95 \%$ confidence limit. Calculations 119 suggest that the branch is actually much smaller $\left(4 \times 10^{-4 \%}\right)$, and in deriving the $f t$-value for the neutron $\beta$-decay we have neglected it.

The statistical rate function with a radiative correction for neutron $\beta$ decay has been calculated by Wilkinson 112. Correcting his result for the current best $Q_{E C}$ value, and combining it with the overall average mean-life quoted in Table 5, we obtain

$$
f t\left(1+\delta_{R}\right)=1054.4 \pm 2.4 s
$$

\subsubsection{Determination of $\lambda$}

To date, the most effective measure of $\lambda$ has proven to be the $\beta$ asymmetry from the decay of polarized neutrons. Such experiments are designed with a $\beta$ detector on the axis of neutron polarization; then spectra are recorded for both polarization directions to yield the asymmetry (see Eq. 77):

$$
\frac{N^{+}-N^{-}}{N^{+}+N^{-}}=\frac{v}{c} P A \cos \theta
$$

in which $N^{ \pm}$is the electron count rate for each direction of neutron polarization, $P$ is 
the magnitude of the polarization, $\theta$ is the angle between the electron momentum and the polarization direction, and $v$ is the electron velocity. In practice, $A$ must also be corrected for the effects $(\sim 1 \%)$ of nuclear recoil and weak magnetism 112 before it can be used to extract a value for $\lambda$ via Eq.78.

The results of five such experiments are listed in Table 5. Three 111, 113,115 required electrons to be observed in coincidence with protons in order to reduce background events, while the other two obviated the need for proton detection by relying in one case 114 on the large volume and high efficiency of the in-line PERKEO spectrometer and in the other 110 on a time-projection chamber operated in coincidence with plastic scintillators to determine the electrons' track and energy, respectively. Since the mid 1980's, the availability of high-flux beams of polarized cold neutrons has helped improve the precision of such measurements.

Only one experiment to determine the $e-\bar{\nu}$ angular-correlation coefficient, $a$, has achieved sufficient precision to contribute to the determination of $\lambda$ (see Table 5). Stratowa et al. 117 measured the energy spectrum of protons recoiling down a tangential reactor beam tube from neutrons decaying in the centre of the reactor. The shape of this spectrum characterizes the correlation between the unobserved decay products: the electron and the antineutrino.

The five measurements of $\lambda$ based on the observed $\beta$-asymmetry are consistent with one another, and with the measurement from the $e-\bar{\nu}$ correlation. The resultant world average is given in Table 5 .

\subsubsection{Implications for the Standard Model}

We are now in a position to extract experimental values for the vector and axialvector coupling constants from neutron decay, compare the former with the result from $0^{+} \rightarrow 0^{+}$nuclear beta decays, and examine the implications for the unitarity test of the CKM matrix (see sect.2.2.4). The current experimental status from neutron decay can be summarized in two expressions,

$$
\begin{aligned}
\lambda=G_{\mathrm{A}}^{\prime} / G_{\mathrm{V}}^{\prime} & =-1.2599 \pm 0.0025 \\
\left(G_{\mathrm{V}}^{\prime 2}+3 G_{\mathrm{A}}^{\prime 2}\right) /(\hbar c)^{6} & =(7.701 \pm 0.018) \times 10^{-10} \mathrm{GeV}^{-4}
\end{aligned}
$$

where the latter follows from combining Eqs.75 and 82. The solution of these equations, shown graphically in figure 2, gives the following results for the effective coupling constants:

$$
\begin{aligned}
& G_{\mathrm{A}}^{\prime} /(\hbar c)^{3}=-(1.4566 \pm 0.0018) \times 10^{-5} \mathrm{GeV}^{-2} \\
& G_{\mathrm{V}}^{\prime} /(\hbar c)^{3}=(1.1561 \pm 0.0023) \times 10^{-5} \mathrm{GeV}^{-2} \quad \text { [neutron] }
\end{aligned}
$$




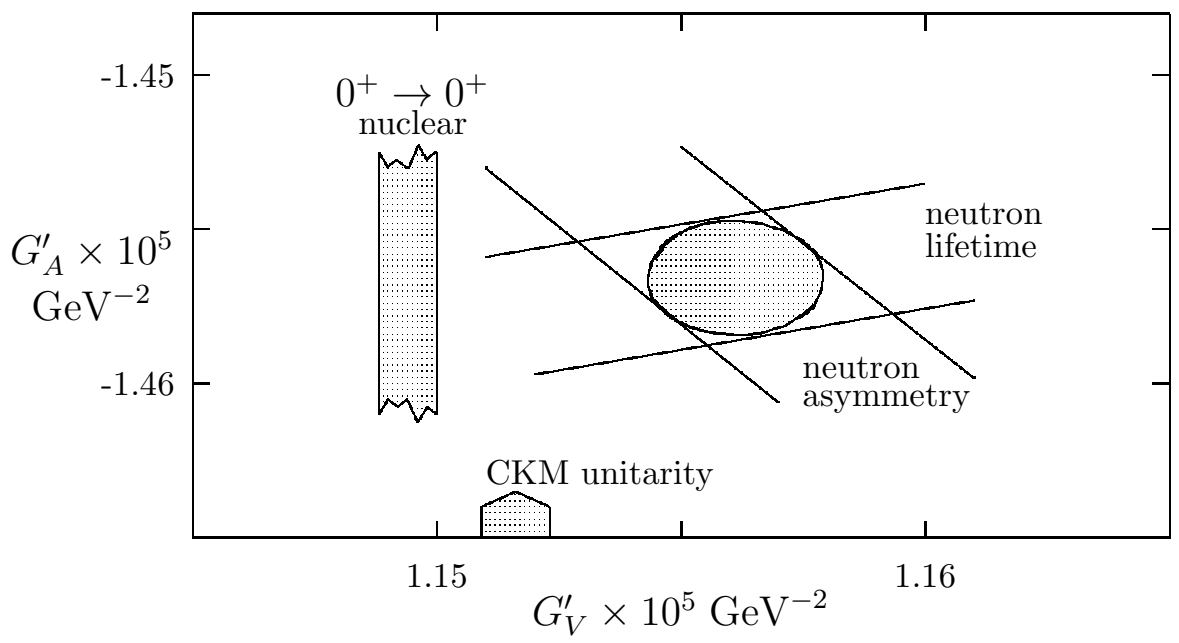

Fig. 2. Allowed regions of $G_{A}^{\prime}$ and $G_{V}^{\prime}$ from neutron decay, nuclear superallowed decays and CKM unitarity

In sect. 2.2 we described the study of $0^{+} \rightarrow 0^{+} \beta$-decay transitions in nine nuclei. The results, shown in Table 2 , led to an average $\overline{\mathcal{F} t}$ value which, with the help of Eqs.27 and 37, becomes:

$$
G_{\mathrm{V}}^{\prime} /(\hbar c)^{3}=(1.1494 \pm 0.0006) \times 10^{-5} \mathrm{GeV}^{-2} \quad\left[0^{+} \rightarrow 0^{+}\right]
$$

where the quoted error bar includes provision for a "systematic" uncertainty in calculating the charge corrections (see sects. 2.2.3.1 and 2.2.4). The neutron result is considerably less precise but, even so, it is in serious disagreement with nuclear decays. The discrepancy is not understood. The analysis of the nuclear results suffers from uncertainties in the effects of charge dependence and nuclear structure; the neutron work is faced with more formidable experimental challenges. There is no reason to suppose, at this time, that either result is in doubt beyond its quoted uncertainty.

Following the approach taken in sect.2.2.4, the neutron result for the vector coupling constant can be used to test the unitarity of the CKM matrix, yielding

$$
\left|V_{u d}\right|^{2}+\left|V_{u s}\right|^{2}+\left|V_{u b}\right|^{2}=1.0077 \pm 0.0040
$$

which differs from unitarity at a confidence level of $\sim 99 \%$, but in the opposite sense to the result from nuclear $\beta$ decay. In the absence of any viable explanation within the Standard Model for the discrepancy between the neutron and nuclear results, nor any 
reason to prefer one over the other, we can do no better than take the weighted average of both results for $G_{\mathrm{V}}^{\prime}$, suitably inflating the resultant uncertainty. This leads to

$$
\begin{aligned}
G_{\mathrm{V}}^{\prime} /(\hbar c)^{3} & =(1.1498 \pm 0.0015) \times 10^{-5} \mathrm{GeV}^{-2} \quad \text { [average] } \\
\left|V_{u d}\right| & =0.9741 \pm 0.0017 \quad \text { [average] }
\end{aligned}
$$

and

$$
\left|V_{u d}\right|^{2}+\left|V_{u s}\right|^{2}+\left|V_{u b}\right|^{2}=0.9978 \pm 0.0037 \quad \text { [average] }
$$

which agrees with unitarity.

\subsection{Muon Capture in Hydrogen}

The induced pseudoscalar coupling constant, $g_{\mathrm{P}}$ of Eq. 10, is the most poorly determined of all the nucleon weak-interaction coupling constants. This is because in semi-leptonic weak processes involving first-generation leptons, such as beta decay or electron capture, the pseudoscalar term gives negligible contribution to the transition rate. Consider the T-matrix involving just the pseudoscalar term:

$$
\begin{aligned}
T_{f i} & =-\frac{G_{\mathrm{F}}}{\sqrt{2}} V_{u d} i \bar{\psi}_{p}\left(i g_{\mathrm{P}} k_{\mu} \gamma_{5}\right) \psi_{n} i \bar{\psi}_{e} \gamma_{\mu}\left(1+\gamma_{5}\right) \psi_{\bar{\nu}_{e}} \\
& =-\frac{G_{\mathrm{F}}}{\sqrt{2}} V_{u d} i \bar{\psi}_{p}\left(-\frac{g_{\mathrm{P}}}{2 M} k_{\mu} \not k \gamma_{5}\right) \psi_{n} i \bar{\psi}_{e} \gamma_{\mu}\left(1+\gamma_{5}\right) \psi_{\bar{\nu}_{e}} \\
& =-\frac{G_{\mathrm{F}}}{\sqrt{2}} V_{u d} i \bar{\psi}_{p}\left(-\frac{g_{\mathrm{P}}}{2 M} \not k \gamma_{5}\right) \psi_{n} i \bar{\psi}_{e} \not k\left(1+\gamma_{5}\right) \psi_{\bar{\nu}_{e}} \\
& =-\frac{G_{\mathrm{F}}}{\sqrt{2}} V_{u d} i \bar{\psi}_{p}\left(\frac{g_{\mathrm{P}} m_{e}}{2 M} \not k \gamma_{5}\right) \psi_{n} \bar{\psi}_{e}\left(1+\gamma_{5}\right) \psi_{\bar{\nu}_{e}},
\end{aligned}
$$

where $k=p_{e}+p_{\nu}$. Here pseudoscalar coupling is transformed to pseudovector coupling in line two, a step valid only for on-shell nucleons. The factor $k_{\mu}$ is moved into the lepton matrix element in line three, where application of the Dirac equation, $\bar{\psi}_{e} \not p_{e}=i m_{e} \bar{\psi}_{e}$, shows immediately that the pseudoscalar term is of size $g_{\mathrm{P}} m_{e} /(2 M)$ and is very small. However, for second-generation leptons participating in such processes as muon capture, the factor becomes $g_{\mathrm{P}} m_{\mu} /(2 M)$ and is much larger.

Consequently, experiments to determine $g_{\mathrm{P}}$ are based on muon capture in hydrogen. The two main processes that contribute to the disappearance of a negative muon stopped in pure hydrogen are muon decay, $\mu^{-} \rightarrow e^{-}+\bar{\nu}_{e}+\nu_{\mu}$, and nuclear capture, $\mu^{-}+p \rightarrow n+\nu_{\mu}$. In the latter process, a $5.2 \mathrm{MeV}$ neutron is emitted. Because the weak interaction is strongly spin dependent, an accurate specification of the initial muonproton state is vital to the interpretation of the experimental results. The history of a negative muon, stopping in a hydrogen target, is as follows. At low velocity, the muon 
is captured by a proton and cascades to the $1 S$ state in less than $10^{-9} \mathrm{~s}$; this neutral $(\mu p)$ atomic system is formed in a statistical mixture of triplet and singlet states. In general, the triplet state converts to the singlet state with a rate depending upon the hydrogen density. Even in gaseous hydrogen, for pressures above five atmospheres, the conversion rate is large compared to the natural decay rate, so nuclear capture occurs predominantly from the singlet state.

In liquid hydrogen, the singlet $(\mu p)$ system further converts rapidly into a $(p \mu p)^{+}$ molecular ion if the muon has not decayed first. Consequently, it is from the stable molecular ion that the muon is most likely to be captured or to decay. Most experiments are able to measure the time of each capture and thus the configuration from which capture takes place is known. Usually only capture events that have originated in a $(p \mu p)$ system are included in the analysis. The $(p \mu p)^{+}$molecular ion is almost invariably formed in the ortho state for which the capture rate, $\Lambda_{o m}$, is given by $2 \gamma_{o}\left(\frac{3}{4} \Lambda_{s}+\frac{1}{4} \Lambda_{t}\right)$, where $\Lambda$ refers the the capture in the triplet and singlet atoms, and $2 \gamma_{o}$ is a wave function overlap factor of the muon with the proton, calculated to be $2 \gamma_{o}=1.01 \pm 0.01$. There is a small probability that the ortho state will decay to the lower-energy paramolecular state before the capture occurs. The capture rate from the para state is given by $\Lambda_{p m}=2 \gamma_{p}\left(\frac{1}{4} \Lambda_{s}+\frac{3}{4} \Lambda_{t}\right)$, with $2 \gamma_{p}=1.15 \pm 0.01$, and is smaller than the ortho rate because $\Lambda_{s} \gg \Lambda_{t}$. Nevertheless, a correction to the measured capture rate has to be applied for this ortho-to-para transition and this correction significantly influences the extraction of the $g_{\mathrm{P}}$ coupling constant.

Early experiments $120,121,122,123$ obtained the capture rate by observing the outgoing 5.2 MeV neutrons. The principal limitation was the calibration of the absolute efficiency of the neutron detectors. A more recent Saclay experiment 124 obtained the capture rate by comparing the lifetime $\tau_{\mu^{-}}$of negative muons stopped in liquid hydrogen with the lifetime $\tau_{\mu^{+}}$of positive muons. Both $\tau_{\mu^{-}}$and $\tau_{\mu^{+}}$were measured by detection of the delayed muon-decay electrons. The capture rate was then obtained from the difference in these two lifetimes. In all cases, the hydrogen in the target had to be highly purified and isotopically pure. If small amounts of deuterium or other atoms are present, then an irreversible transfer to the deuteron (or heavier atom) is likely. Use of pure hydrogen, therefore, is very important to minimize this loss. The Columbia and Saclay experiment,120,121, 124 used liquid hydrogen targets; the capture rates were corrected for the ortho-to-para transition, which was explicitly measured in a subsequent Saclay experiment125. The Dubna and CERN experiments 122,123 used gas targets.

The resulting values of the dimensionless quantity $m_{\mu} g_{\mathrm{P}} / g_{\mathrm{A}}$ from these five experiments are: $4.8 \pm 6.3$ (ref. .120$), 8.7 \pm 3.7$ (ref.121), $8.2 \pm 3.1$ (ref. 122 ), $6.3 \pm 4.7$ (ref. 123 ) and $5.6 \pm 2.6$ (refs. 124 ). The average is $6.9 \pm 1.5$ in excellent agreement with the PCAC value of 6.7 from Eq.74, evaluated with $k^{2}=0.88 m_{\mu}^{2}$, which is appropriate for hydrogen. Were it not for the ortho-to-para correction, the value from the Saclay experiment would have been $10.6 \pm 1.6$ rather than the quoted $5.6 \pm 2.6$ ! A new measurement of the ortho-to-para transition rate with improved accuracy would be very desirable. 
Despite the good agreement between the ordinary muon capture (OMC) experiments and the PCAC prediction, it should be noted that the OMC rate is insensitive to the exact value of $g_{\mathrm{P}}$ since an individual measurement, based on a $4 \%$ OMC rate determination at Saclay 124 , gives a $42 \%$ uncertainty for $g_{\mathrm{P}}$. This is because, in liquid hydrogen, $80 \%$ of the capture takes place from the ortho-molecular state, which is predominantly the atomic singlet state. In contrast, in radiative muon capture (RMC), in which $\mathrm{OMC}$ is accompanied by a bremsstrahlung photon, the photon carries away one unit of angular momentum and the capture is mainly from the triplet atomic state. The triplet capture rate is 15 times more sensitive than the singlet rate to the value of $g_{\mathrm{P}}$. Beder and Fearing 126 have estimated that an $8 \%$ measurement of the RMC rate could provide a $10 \%$ value for $g_{\mathrm{P}}$.

The experimental problems in measuring RMC in hydrogen are formidable. The partial RMC branching ratio (for $E_{\gamma} \geq 57 \mathrm{MeV}$ ) is only $1.6 \times 10^{-8}$ and the bremsstrahlung from radiative muon decay or from the Michel electrons is nearly five orders of magnitude larger. Consequently, the apparatus requires good energy resolution and an accurate knowledge of the high-energy tail of the response function in order to guarantee that the RMC signal region is not contaminated by events from the bremsstrahlung region. The low branching ratio also requires an extremely high purity level in the hydrogen liquid itself; otherwise the $\mu^{-}$will be lost from the proton before the capture can take place. Impurity levels of $\leq 10^{-9}$ for all elements other than hydrogen are necessary since the capture probability increases as $Z^{4}$. An experiment 127 presently underway at TRIUMF hopes to achieve an $8 \%$ measurement of the RMC capture rate.

\section{Axial-vector Interaction in Nuclei}

\subsection{Quenching of the axial-vector current in nuclei}

Since the vector current is a conserved current, its normalization is unchanged from one environment to another: $g_{\mathrm{v}}(0)$ is unity at the quark level, at the singlenucleon level and in finite nuclei. In contrast to this, the axial current is not conserved and its normalization therefore becomes environment dependent: $g_{\mathrm{A}}(0)$ is unity at the quark level, is greater than unity (of order 1.26) at the single-nucleon level and reduced relative to 1.26 in finite nuclei. The degree of reduction, however, is nucleus dependent. Since pions cause the failure of the axial current to be conserved, as evidenced in the PCAC hypothesis (see Eq. 60), and since nucleons in nuclei are interacting by the exchange of pions, it is not surprising that the axial current in nuclei is modified. One mechanism frequently discussed in this context involves the $\Delta(1232, s=3 / 2, t=$ $3 / 2$ )-isobar, which can be viewed as an internal spin-isospin-flip excitation of a single nucleon. A nucleon, for example, in emitting a pion can be raised to its $\Delta$-excited state. The $\Delta$ is subsequently de-excited back to the nucleon through an interaction with the axial current, while the pion is absorbed by a second nucleon. This and related processes are calculable in perturbation theory but the results are strongly model dependent, relying on assumptions made concerning the isobar's interactions. It is probably worth repeating here the cautionary remarks of Lipkin and Lee 128 : "There 
has been considerable debate of the possibility that isobars may be present in nuclei. However, it is not clear exactly what this means. The isobar is a highly unstable particle with a natural width much larger than the spacing between nuclear levels. It is, therefore, unclear whether an isobar present in a nucleus can be considered as an elementary fermion in some approximation, as a three-quark composite, or as a pionnucleon resonance. Furthermore, an isobar present in a normal nuclear ground state is very far off shell, and it is not clear how to extrapolate the properties of such a complicated object far off shell. All this confuses the issue and makes it difficult to test experimentally for the presence of such isobars."

The idea that the axial-vector coupling constant might be renormalized for nucleons embedded in a nucleus was first discussed by Ericson et al. 129 . They pointed out that the pion field within the nucleus might be suppressed at small momenta because of the strong short-range repulsion between nucleons in the medium. Thus, an axial-vector $\beta$-decay in nuclei would, using the arguments of PCAC, be similarly suppressed. This was worked out in more detail by Rho 130 and Ohta and Wakamatsu 131 , who showed that the $\Delta$-isobar would be the main mediator in effecting such a suppression. Experimental data, as we will shortly discuss, also give evidence for this suppression, but whether or not it is due to the influences of $\Delta$-isobars is not so clear. There are other many-body corrections to be applied to the nuclear-structure calculations, which arise from short-range central and tensor correlations between nucleons and which also lead to a suppression of axial-vector rates 132,133 .

One of the primary sources of experimental information on axial-vector transitions in nuclei comes from nuclear beta decay. Referring to the axial current given in Eq.12, we ignore second-class currents and note that the pseudoscalar coupling term gives a negligible contribution. Thus, only the $i \bar{\psi} g_{\mathrm{A}} \gamma_{\mu} \gamma_{5} \frac{1}{2} \tau^{a} \psi$ term is operable. In nuclear physics it is sufficiently accurate to consider a nonrelativistic limit of this current in which only leading terms in $p / M$, where $p$ is the nucleon momentum and $M$ its mass, are retained and four-component Dirac spinors, $\psi$, are replaced by two-component Pauli spinors, $\chi$. The leading term arises when the Lorentz index, $\mu$, is space-like:

$$
\mathbf{A}^{h}=-g_{\mathrm{A}} \chi^{\dagger} \boldsymbol{\sigma} \frac{1}{2} \tau^{a} \chi
$$

where $\boldsymbol{\sigma}$ is the Pauli spin operator and $\tau^{a}=\tau^{1} \pm i \tau^{2}$, with the upper sign for $\beta^{-}$ transitions and the lower sign for $\beta^{+}$transitions. The quantity between $\chi^{\dagger} \chi$ is taken as a one-body operator, the matrix elements of which are evaluated in the many-body nucleus with shell-model wavefunctions. The experimentally determined quantity, the $f t$-value, for a pure axial-vector transition is related to this matrix element via ( $c f$. $\mathrm{Eq} \cdot 75$

$$
f t\left(1+\delta_{R}\right)=\frac{6146 \pm 6}{g_{\mathrm{A}}^{2} B(G T)} \mathrm{s}
$$

where 


$$
\begin{aligned}
B(G T) & =\left\langle M_{\mathrm{A}}\right\rangle^{2} \\
\left\langle M_{\mathrm{A}}\right\rangle & =\frac{\hat{J}_{f}}{\sqrt{2} \hat{J}_{i}}\left\langle T_{i} T_{z i} 1 \mp 1 \mid T_{f} T_{z f}\right\rangle\left\langle J_{f} T_{f}\|\boldsymbol{\sigma} \boldsymbol{\tau}\| J_{i} T_{i}\right\rangle .
\end{aligned}
$$

Here the spin, isospin and $z$-projection of isospin are denoted $J_{i}, T_{i}$ and $T_{z i}$ for the initial state and $J_{f}, T_{f}$ and $T_{z f}$ for the final state. The triple-barred matrix element is a reduced matrix element in both spin and isospin spaces in the notation of Brink and Satchlere34 and $\hat{J}=(2 J+1)^{1 / 2}$. The constant, $6146 \pm 6 \mathrm{~s}$, in Eq. 93 is given by $2 \overline{\mathcal{F} t}$, where $\overline{\mathcal{F} t}$ is the average electromagnetically-corrected $f t$ value for the superallowed Fermi $0^{+} \rightarrow 0^{+}$beta transitions (see Eq. 39).

The operator $\boldsymbol{\sigma} \boldsymbol{\tau}$, known as the Gamow-Teller (GT) operator, has no dependence on orbital angular momentum and hence has no non-zero matrix elements between states of different orbital symmetry. In particular, in a shell-model calculation carried out in a complete basis of harmonic oscillator states (e.g. a $\left(0 p_{3 / 2}, 0 p_{1 / 2}\right)$-basis for $p$-shell nuclei or a $\left(0 d_{5 / 2}, 1 s_{1 / 2}, 0 d_{3 / 2}\right)$-basis for $s d$-shell nuclei) the GT-operator acting on any member of the basis connects only to other states within the basis and not to any state with occupancy in a different major oscillator shell. Complete untruncated shell-model calculations within a single major oscillator shell are called $0 \hbar \omega$ calculations. A working definition for the term quenching is as follows: for beta decays populating well-defined, isolated states in the daughter nucleus, the ratio of the experimentally measured rate to the calculated rate in a full $0 \hbar \omega$ calculation is called the quenching factor. An average quenching factor implies an average over many transitions. The definition is model dependent in the sense that the shell-model calculation is dependent on the choice of residualinteraction, but for an average value it is hoped that this dependence is not severe. $\#$ Equivalently, one can discuss a quenching in terms of a renormalization of the axial-vector coupling constant. We define

$$
g_{\mathrm{A}, \mathrm{eff}}=g_{\mathrm{A}}+\delta g_{\mathrm{A}},
$$

where $g_{\mathrm{A}}$ is the free-nucleon value of 1.26 and $\delta g_{\mathrm{A}}$ the correction to it; thus, the quenching factor is $\left(g_{\mathrm{A}, \text { eff }} / g_{\mathrm{A}}\right)^{2}$.

The first systematic attempt to determine an average quenching factor, $q$, was by Wilkinson 136 from a study of $\beta$-transitions in the $p$-shell and the lower $s d$-shell nuclei ( $A=17-21$ ), in which he obtained $q=0.80 \pm 0.06$. A more comprehensive analysis by Brown and Wildenthal137 using full $0 \hbar \omega$ calculations throughout the $s d$-shell for 189 data, for which the experimental error on the deduced GT matrix element was $10 \%$ or less, found an average quenching factor of $q=0.63 \pm 0.01$; the quoted error here is limited to the statistical error on the fit. Significant quenching seems to be evident. One configuration has a special role to play here, for example in the ground states of

\footnotetext{
$\overline{\# 5}$ However, see some contrary remarks by Brown 135 in a discussion of the $\beta$-decay of ${ }^{37} \mathrm{Ca}$.
} 
$A=15,17,39$ and 41 nuclei: it is a closed-LS-shell-plus (or minus)-one nucleon. In these cases, the full $0 \hbar \omega$ calculation involves just a single-particle matrix element, so microscopic calculations can seek to evaluate corrections to the single-particle matrix element and identify the origin of the quenching phenomenon. The Tokyo 132, 138 and Chalk River 139 . 140 groups have attempted this with similar results.

Generally, the quenching stems either from the inadequacy of the single-particle description of the nuclear state or from the inadequacy of the one-body GT operator. Calculations of the first effect, frequently called core polarization, estimate through perturbation theory the admixtures of $2 p$ - $1 h$ and $3 p$ - $2 h$ configurations in the basically single-particle wave function and evaluate the impact the admixtures have on the calculated matrix element. At LS closed shells there is no contribution to the core polarization in first order because the one-body GT operator has no spatial dependence and thus cannot excite a $1 p$ - $1 h$ state from a closed LS shell. Therefore, calculations must be taken to second order in perturbation theory. This leads to a time-consuming calculation, as there is no selection rule to limit the intermediate-state summation and the convergence is slow. This is particularly true with tensor forces in the residual interaction as first stressed by Shimizu et al.132. This propensity of the tensor force to couple strongly to high-lying states has been called 'tensor correlations' and the phenomenon leads to a reduction in the GT matrix element.

The other inadequacy concerns the use of one-body operators. Corrections arise because nucleons in nuclei interact through the exchange of mesons, and this exchange can be perturbed by the action of the weak axial current. Since this perturbation requires at least two nucleons to be involved, corrections of this type lead to twobody GT operators. The possible pion-exchange processes can be classified into two types: Born graphs, involving only nucleons and pions, and non-Born graphs in which the axial current either excites a nucleon to its isobar $\Delta$ state, or converts the pion into a heavy meson, such as the $\rho$. The important point is that for axial currents the pion Born graphs are identically zero 141 . For the non-Born graphs, we can distinguish between diagrams involving nucleon excitations - referring to these as isobar graphs and those in which a pion is converted to a $\rho$ meson - referring to these as $\rho \pi$ or mesonexchange-current (MEC) graphs. In general, the MEC graphs give a small contribution and are not an important ingredient in the quenching of the GT matrix element.

There are two further points to consider. The core-polarization calculation corrects the matrix element of a one-body operator evaluated in the closed-shell-plus-one configuration for the presence of $2 p$ - $1 h$ and $3 p$ - $2 h$ admixtures in the single-particle wave function. The perturbation calculation is carried out to second order in the residual interaction (or to the fourth power in the meson-nucleon coupling constants). It is logical, therefore, that the matrix elements of two-body operators should likewise be corrected for $2 p-1 h$ and $3 p-2 h$ admixtures. Since the two-body operator itself involves the meson-nucleon coupling constants to the second power, it is sufficient to estimate this correction to first order in the residual interaction. These terms have been called 'crossing terms' in the work of the Tokyo group 138. The second point concerns the one- 
Table 6. Corrections to the ground-state diagonal and spin-flip off-diagonal matrix elements, $\delta\left\langle M_{A}\right\rangle$, expressed as a percentage of the single-particle value, $\left\langle M_{A}\right\rangle_{s p}$, for closed-shell-plus-one configurations at $A=17$ and $A=41$. Quenching factors, $q$, are also given.

\begin{tabular}{lcccc}
\hline & \multicolumn{4}{c}{$\delta\left\langle M_{A}\right\rangle /\left\langle M_{A}\right\rangle_{s p}$ in $\%$} \\
\cline { 2 - 5 } & $d_{5 / 2} \rightarrow d_{5 / 2}$ & $d_{5 / 2} \rightarrow d_{3 / 2}$ & $f_{7 / 2} \rightarrow f_{7 / 2}$ & $f_{7 / 2} \rightarrow f_{5 / 2}$ \\
\hline Core polarization & -9.0 & -11.3 & -11.8 & -14.1 \\
Isobars & -0.9 & -5.9 & -2.1 & -6.2 \\
MEC & -1.2 & 0.3 & -1.4 & 0.2 \\
Crossing terms & 3.0 & 2.3 & 3.4 & 2.6 \\
Relativistic corr & -2.1 & -1.2 & -2.3 & -1.3 \\
Total & -10.2 & -15.8 & -14.2 & -18.8 \\
Quenching factor & 0.81 & 0.71 & 0.74 & 0.66 \\
Experiment, $q$ & $0.74 \pm 0.01$ & \multicolumn{5}{c}{$0.54 \pm 0.01$} \\
\hline
\end{tabular}

body GT operator, which is obtained as a leading term in the nonrelativistic reduction of a relativistic axial-vector current. There is a few per cent correction coming from the next-order terms in the reduction.

In summary, then, corrections to lowest-order shell-model estimates of the GT matrix element come from: core polarization, isobar currents, MEC currents, crossing terms and relativistic corrections. All these ingredients for closed-LS-shell-plus-one nuclei have been calculated by Arima et al. 138 and by Towner and Khanna 139 . We quote in Table 6 some results from a review by Towner 40 , in which more details can be found.

Note that the calculated quenching factors, although in the expected range of 0.66 to 0.81 , are higher than the experimental values for the diagonal matrix elements and, for the $0 d$ orbits, are also higher than the average quenching factor of $0.63 \pm 0.01$ determined by Brown and Wildenthal 137. Furthermore, there is more quenching evident in spin-flip than in diagonal matrix elements, with approximately two-thirds of the quenching coming from core polarization (notably tensor correlations) and one-third from isobars.

\section{2. $(p, n)$ and $(n, p)$ reactions}

The reactions $(p, n)$ and $(n, p)$ are comparable to $\beta^{-}$and $\beta^{+}$decay, respectively, but are not limited by the energetics of a radioactive decay. In studying these reactions, it is useful to define a Gamow-Teller strength function by the relation:

$$
S(\omega)=\sum_{f}\left\langle M_{\mathrm{A}}(i \rightarrow f)\right\rangle^{2} \delta\left(E_{f}-E_{i}-\omega\right),
$$

where $\omega$ is excitation energy, $\delta$ is a resolution function, and $i$ and $f$ represent the initial 
and final nuclear eigenstates of energies $E_{i}$ and $E_{f}$. The major peaks in $S(\omega)$ are identified with a collective state: the Gamow-Teller giant resonance (GTR). Unfortunately, beta decay has access to nuclear states in a very limited energy window and misses the strongest states in the strength function. Therefore, to map out the complete strength function one needs probes that allow the independent variation of both energy transfer and momentum transfer to the target. This is possible with hadronic probes such as the $(p, n)$ and $(n, p)$ charge-exchange reactions.

The Gamow-Teller resonance has quantum numbers $J^{\pi}=1^{+}, L=0, S=1, T=1$ indicating it is populated through spin- and isospin-excitations. In the $(p, n)$ reaction this mode is reached via the $V_{\sigma \tau} \boldsymbol{\sigma}_{p} \cdot \boldsymbol{\sigma}_{j} \boldsymbol{\tau}_{p} \cdot \boldsymbol{\tau}_{j}$ component of the interaction between the projectile and target nucleons. The labels $p$ and $j$ refer to the projectile and the struck nucleon respectively. With respect to the target, the $(p, n)$ probe has a similar spin-isospin operator structure as the GT operator of $\beta$-decay. However, the $(p, n)$ reaction simulates the $\beta$-decay conditions only if the cross-section is measured at very small momentum transfer. In the $(p, n)$ reaction, this condition can be met only for $0^{\circ}$ scattering and at high bombarding energies. As there is no orbital excitation in a GT transition, the angular distribution for the $(p, n)$ reaction to the GTR will have a characteristic $L=0$ shape, with the maximum cross-section at zero degrees.

The GTR occurs at a higher excitation energy than the isobaric analogue of the target ground state (IAS), which carries the quantum numbers $J^{\pi}=0^{+}, L=0, S=$ $0, T=1$. The $(p, n)$ reaction excites the IAS through the force component $V_{\tau} \boldsymbol{\tau}_{p} \cdot \boldsymbol{\tau}_{j}$, which requires no spin flip $(S=0)$. While the IAS is the dominant peak in the spectrum at low incident energies $\left(E_{p} \leq 100 \mathrm{MeV}\right)$, it is completely swamped 42 by the large GT cross-section at $E_{p}=200 \mathrm{MeV}$. The large difference in the excitation strengths of the IAS and GTR as a function of the incident projectile energy is due to the strong energy dependence of the $V_{\tau}$-term in the projectile-target-nucleon interaction. The force strength $V_{\tau}$ is greatly reduced with increasing projectile energy, while the force strength $V_{\sigma \tau}$ is nearly independent of incident energy.

The $(p, n)$ reaction is a powerful spectroscopic tool for measuring the GT strength functions of nuclei. The zero-degree $(p, n)$ cross-section can be calculated in a distortedwave theory and compared to experiment to extract the spectroscopic information: namely $B(G T)$ of Eq.94. However, there is sufficient uncertainty in the distortedwave theory, especially with regard to its absolute normalization, that it is usual to calibrate the theory by measuring the $(p, n)$ cross-sections of specific transitions for which the $B(G T)$ values are already known from $\beta$-decay 143 . Once this is done, then the $(p, n)$ reaction can be used to determine the GT matrix elements of states that cannot be populated by $\beta$-decay. In particular, it is possible to map out the whole GT response function in the zero-degree spectrum. There are, however, other states with multipolarities $L>0$, which also contribute to the $0^{\circ}(p, n)$ cross-section. In general, these states receive their maximum cross-sections at larger scattering angles, but their angular distributions extend forward to $0^{\circ}$. These states lead to a background contribution that has to be subtracted from the data. A discussion of the theoretical 
techniques for doing so can be found in the papers of Osterfeld 144.145.

The breakthrough in the use of the $(p, n)$ reaction to study GT resonances came in 1980 from experiments at the Indiana University Cyclotron Facility. These experiments 146, 147, 148, 149.150 demonstrated the existence of very collective spin-isospin modes in nuclei. An excellent review of these and the many subsequent experiments is to be found in ref. 142. The spectra of nuclei with neutron excess are seen to be dominated by one prominent peak, which is interpreted as the giant Gamow-Teller resonance. This collective mode was already predicted by Ikeda, Fujii and Fujita151, who inferred the existence of the GTR from the absence of spin-isospin strength at low excitation energies. Their argument is based on a sum rule. Let us integrate the strength function of Eq.96 to define a sum:

$$
S=\int S(\omega) d \omega=\sum_{f}\left\langle M_{\mathrm{A}}(i \rightarrow f)\right\rangle^{2} .
$$

Then, from the commutator algebra of isospin, $\left[\tau^{x}, \tau^{y}\right]=2 i \tau^{z}$, we obtain for the difference in the sum rule for $\beta^{-}$and $\beta^{+}$emissions from a given nucleus the modelindependent result 151 :

$$
S\left(\beta^{-}\right)-S\left(\beta^{+}\right)=3(N-Z) .
$$

That is, the difference in the sums for the $(p, n)$ and $(n, p)$ reactions on a particular target is quite independent of nuclear structure. In heavy nuclei with large neutron excess, $S\left(\beta^{+}\right) \simeq 0$, because all states into which a proton could be transferred within the same major shell are already fully occupied: the GT transition is Pauli blocked. Setting $S\left(\beta^{+}\right)=0$ then gives a lower limit for the $\beta^{-}$sum: viz.

$$
S\left(\beta^{-}\right) \geq 3(N-Z)
$$

At intermediate energies $\left(E_{p} \sim 100-200 \mathrm{MeV}\right)$, the selective excitation of the GT giant resonance by the $(p, n)$ reaction has been observed in almost the entire periodic table. The striking result 149 is that experimentally only $(60 \pm 10) \%$ of the expected sum-rule limit, $3(N-Z)$, can be located in the excitation region where the major GT peaks occur. This means that at least $40 \%$ of the GT strength is unobserved in the spectra. As described in the previous section for beta decay, this missing GT strength has been interpreted as evidence for isobar admixtures in the nucleonic wave function 129.130 .153 or, alternatively, as indicating 133 that high-lying $2 p$ - $2 h$ states mix with the low-lying $1 p-1 h$ GT states and shift GT strength above and below the GTR. If this latter mechanism is the right one, then the so-called missing GT strength would actually be located in the background. The detection of this strength would not be an easy task because the spectrum on the high-energy side of the GTR is continuous and structureless, making it difficult to extract information. Calculations by Smith and Wambach 154 indicate that significant strength from $2 p-2 h$ mixing resides in the tails of the resonances, suggesting 
that the quenching due to $\Delta$-isobars is $10-20 \%$. This is a further indication that quenching due to $\Delta$ 's is small.

In conclusion we note two recent experiments that have elicited an exchange of comments in the literature:

- A group 155 at TRIUMF has measured angular distributions of the ${ }^{54} \mathrm{Fe}(p, n){ }^{54} \mathrm{Co}$ and ${ }^{54} \mathrm{Fe}(n, p){ }^{54} \mathrm{Mn}$ cross-sections at incident beam energies of $300 \mathrm{MeV}$ to test the GT sum rule, Eq.98, for a case in which the GT strength is strong in both channels. The $(p, n)$ cross-section at $0^{\circ}$ for $L=0$ was summed up to an excitation energy of $E_{x}=15 \mathrm{MeV}$. The strength above this excitation energy was not included because of increasing uncertainties in removing dipole and higher multipole strength at higher energies. The integrated strength below $E_{x}=15$ $\mathrm{MeV}$ is $S\left(\beta^{-}\right)=7.5 \pm 1.2$ in agreement with earlier result 156 at $160 \mathrm{MeV}$ of $S\left(\beta^{-}\right)=7.8 \pm 1.9$. The $0^{\circ}(n, p)$ cross-section was summed up to $E_{x}=10 \mathrm{MeV}$, the same criteria being used to decide the upper limit of the integration. The integrated strength is $S\left(\beta^{+}\right)=3.1 \pm 0.6$. A more recent experiment 157 at a lower energy of $97 \mathrm{MeV}$ finds $3.5 \pm 0.3 \pm 0.4$, the first uncertainty being due to statistics and multipole decomposition, the second due to uncertainties in normalization. The sum rule difference in the TRIUMF data thus yields

$$
S\left(\beta^{-}\right)-S\left(\beta^{+}\right)=(7.5 \pm 1.2)-(3.1 \pm 0.6)=4.4 \pm 1.3
$$

to be compared with the model-independent value of $3(N-Z)=6$ in this case. The result is consistent with the quenching factor of 0.6 seen throughout the periodic table in $(p, n)$ reactions where the $(n, p)$ strength is strongly Pauli blocked. However, Goodman et al. 158 have commented that this work does not test the model-independent sum rule because both measured strength functions are incomplete, there being additional strength undetected either because it lies outside the measured energy domain or because it is hidden in the background.

- A recent beta-decay experiment by García et al. 159 has extended the measured GT strength function in the $\beta^{+}$decay of ${ }^{37} \mathrm{Ca}$ up to $8 \mathrm{MeV}$ excitation in the daughter nucleus. Delayed protons were detected in coincidence with gamma rays to identify proton decays of ${ }^{37} \mathrm{~K}$ daughter levels to excited states in ${ }^{36} \mathrm{Ar}$. Some 43 previously undetected proton groups were observed yielding an integrated $B(G T)$ twice as large as that observed previously. In terms of the sum-rule limit, the experiment observed a summed GT strength of 1.95 compared to the sumrule requirement of at least 9 units. Because ${ }^{37} \mathrm{Ca}$ is the isospin mirror of ${ }^{37} \mathrm{Cl}$, it is possible to compare the strength seen in this decay with that measured in the ${ }^{37} \mathrm{Cl}(p, n){ }^{37}$ Ar reaction studied by Rapaport et al. ${ }^{160}$. García et al. made such a comparison and concluded that the $\beta^{+}$decay contains $50 \%$ more GT strength in this energy region than the $(p, n)$ experiment. 
This conclusion led to a flurry of correspondence161, 162. Two issues were raised. First, in the analysis of the $(p, n)$ data, insufficient Gamow-Teller strength was assigned near the isobaric analogue state, which in the reaction is populated by both Fermi and GT operators. Second, the beta-decay data for the low-lying states have to take account of both delayed-gamma and delayed-proton emission 163 .

The current situation is that the ratio of $B(G T)$ values determined from betadecay and $(p, n)$ experiments show a state-by-state scatter with discrepancies in the $B(G T)$ values that can vary by as much as a factor of two. However, these states are below $8 \mathrm{MeV}$ excitation and their $B(G T)$ values are very small. Discrepancies of this size, therefore, are not necessarily surprising because, for weak transitions with $B(G T) \ll 1$, one can expect inadequacies in the reaction model used to extract $B(G T)$ values from forward-angle charge-exchange crosssections. The importance of the $A=37$ example is that it allows a comparison of GT strength inferred from $(p, n)$ experiments and beta-decay experiments over a significantly larger excitation energy range and for significantly more transitions than any other system studied so far. Further experiments are underway.

Despite the large number of GT transitions identified in this example, the $\beta^{+}$ experiment found only $2 / 9$ of the sum-rule limit. Brown 135 points out that if experiment and theory are compared over an energy region that only exhausts a small fraction of the sum rule, the results are very sensitive to the choice of residual interaction used in the shell-model calculations. Some older interactions reproduced the data better than the preferred Wildenthal interaction 164 . This, claims Brown, indicates a need for further improvement in the $s d$-shell Hamiltonian.

\subsection{Radiative Muon Capture in Nuclei}

It was explained in sect. 3.3 that muon-capture data provide the best opportunity to measure the induced pseudoscalar coupling constant. Data on ordinary muon capture (OMC) by the proton yield a value consistent with the PCAC hypothesis, but the accuracy is not sufficient to provide a demanding test of the prediction. Radiative muon capture (RMC) potentially provides greater sensitivity, but the low total capture probability in hydrogen $\left(\sim 10^{-3}\right)$ combined with the low expected branching ratio RMC/OMC $\left(\sim 10^{-5}\right)$ has so far precluded a measurement of the elementary radiative capture process, although results from an experiment 127 at TRIUMF are anticipated soon.

RMC measurements are easier for heavier nuclei, in which the muon capture probability is greatly enhanced: roughly by a factor of $Z^{4}$, where $Z$ is the charge number of the capturing nucleus. Exclusive nuclear RMC to a specific final nuclear state has not yet been attempted and would pose formidable experimental challenges. All measurements of RMC to date have been inclusive ones, in which the final nuclear 
states are not experimentally resolved. These suffer the disadvantage that the nuclear response has to be calculated from some theory and this introduces considerable model dependence.

Most nuclear RMC calculations use the impulse approximation, in which the amplitude for the elementary process is summed incoherently over all the nucleons in the nucleus. Even within the impulse approximation, the calculation is non-trivial, since the RMC amplitudes must be summed over all final states. The earliest calculations adopted the closure approximation 165,166,167 for this final-state summation, in which a single average excitation energy with a corresponding maximum photon energy, $k_{\max }$, is assigned to the final nucleus. The RMC photon spectrum then takes a polynomial form,

$$
\frac{d \Lambda}{d E_{\gamma}} \simeq\left(1-2 x+2 x^{2}\right) x(1-x)^{2}
$$

where $x=E_{\gamma} / k_{\max }$ and $E_{\gamma}$ is the photon energy. The use of the closure approximation has been criticized 168 and, indeed, such calculations typically overestimate the RMC branching ratio by large factors 169 . Nevertheless, the polynomial form given in Eq. 101 usually reproduces the data fairly well provided $k_{\max }$ is treated as a variable parameter. Christillin, Rosa-Clot and Servadio 170 were able to avoid the closure approximation for medium-heavy nuclei by integrating over the nuclear response obtained in a nonrelativistic Fermi-gas model. The only free parameter was the effective nucleon mass, $M^{\star}$, which was fixed at $M^{\star}=0.5 M$ from a global fit to experimental OMC rates. The RMC rate was then calculated in a consistent manner. A recent calculation by Fearing and Welsh 171 cast some doubt on the validity of the nonrelativistic Fermi-gas model calculations. They adopt a fully relativistic Fermi-gas model along with the local-density approximation and realistic nuclear-density distributions and a number of other refinements; they find values for the OMC and RMC rates that are different by factors of two or more. This issue remains unresolved.

In lighter nuclei, a more microscopic account of the response function can be attempted. Christillin 168 uses a phenomenological nuclear excitation spectrum for ${ }^{40} \mathrm{Ca}$ consisting of Lorentzian giant dipole and quadrupole components. The widths and relative strengths are adjusted to reproduce existing photoabsorption and OMC data. A similar approach is used by Christillin and Gmitro ${ }^{172}$ for ${ }^{16} \mathrm{O}$ except explicit account is taken of low-lying states in the ${ }^{16} \mathrm{~N}$ final nucleus in addition to the giant dipole and quadrupole terms. Lastly, Roig and Navarro 173 have invoked SU(4) symmetry and applied sum-rule techniques to calculate $\mathrm{RMC}$ on ${ }^{12} \mathrm{C},{ }^{16} \mathrm{O}$ and ${ }^{40} \mathrm{Ca}$. The branching ratios predicted are substantially lower than those provided by either the phenomenological or microscopic models.

In Table 7 we tabulate the branching ratio and $g_{\mathrm{P}}$ values extracted from RMC measurements at PSI 175 and at TRIUMF 176.177 with various theories for the nuclear response functions. It is evident that the observed branching ratio is falling for heavier nuclei. It also appears that $g_{\mathrm{P}}$ is being quenched but this result is heavily dependent 
Table 7. Branching ratios and pseudoscalar coupling constants extracted from RMC measurements at PSI and at TRIUMF with various theoretical calculations of the nuclear response function.

\begin{tabular}{|c|c|c|c|c|c|c|}
\hline \multirow{2}{*}{ Target } & \multirow{2}{*}{$\begin{array}{c}\text { Branching } \\
\text { Ratio } \\
\times 10^{-5}\end{array}$} & \multicolumn{4}{|c|}{$m_{\mu} g_{\mathrm{P}} / g_{\mathrm{A}}$} & \multirow{2}{*}{ Reference } \\
\hline & & $\mathrm{CRS} 80^{a}$ & $\mathrm{CG} 85^{b}$ & GOT $86^{c}$ & $\mathrm{RN}{ }^{d}$ & \\
\hline \multirow[t]{3}{*}{${ }^{12} \mathrm{C}$} & $2.7 \pm 1.8$ & $7.2_{-11.8}^{+5.6}$ & $9.5 \pm 7.2$ & & & 175 \\
\hline & $2.16 \pm 0.24$ & & $7.1 \pm 0.9$ & & & 176 \\
\hline & $2.13 \pm 0.24$ & & & $13.1_{-2.0}^{+1.8}$ & & 176 \\
\hline \multirow[t]{3}{*}{${ }^{16} \mathrm{O}$} & $2.44 \pm 0.47$ & $6.0_{-3.0}^{+2.0}$ & $8.4 \pm 1.9$ & & & 175 \\
\hline & $2.22 \pm 0.23$ & & $7.3 \pm 0.9$ & & & 176 \\
\hline & $2.18 \pm 0.21$ & & & $13.6_{-1.9}^{+1.6}$ & & 176 \\
\hline${ }^{27} \mathrm{Al}$ & $1.83_{-0.25}^{+0.58}$ & $2.5_{-1.7}^{+3.9}$ & & & & 175 \\
\hline \multirow[t]{3}{*}{${ }^{40} \mathrm{Ca}$} & $2.30 \pm 0.21$ & $6.3_{-1.5}^{+1.0}$ & & & & 175 \\
\hline & $2.07 \pm 0.14$ & & & $5.0 \pm 1.7$ & & 177 \\
\hline & $2.09 \pm 0.19$ & & & & $7.8 \pm 0.9$ & 177 \\
\hline${ }^{\text {nat }} \mathrm{Fe}$ & $1.71 \pm 0.17$ & $3.0 \pm 1.3$ & & & & 175 \\
\hline Mo & $1.26 \pm 0.10$ & $0.0_{-4.1}^{+1.6}$ & & & & 177 \\
\hline Sn & $1.03 \pm 0.08$ & $0.1_{-7.5}^{+1.4}$ & & & & 177 \\
\hline${ }^{165} \mathrm{Ho}$ & $0.75 \pm 0.13$ & $-0.5 \pm 1.4$ & & & & 175 \\
\hline $\mathrm{Pb}$ & $0.60 \pm 0.05$ & $\leq 0.2$ & & & & 177 \\
\hline${ }^{209} \mathrm{Bi}$ & $0.62 \pm 0.08$ & $0 . \overline{2} \pm 1.1$ & & & & 175 \\
\hline
\end{tabular}

${ }^{a}$ ref. 170; ${ }^{b}$ ref. 172 ; ${ }^{c}$ ref. 174; ${ }^{d}$ ref. 173

on the theory of the nuclear response, which is unsatisfactory at the present time.

In light nuclei, there are a few OMC experiments to explicit final nuclear states that lead to a determination of the pseudoscalar coupling constant. To isolate the pseudoscalar term from a term involving the regular axial-vector current requires either two complementary measurements on the same transition or one measurement and a calculation. Further, with the pseudoscalar term identified, a calculation of the matrix element is required to determine the coupling constant. We cite three examples:

- One favoured case is the $\mu^{-}+{ }^{12} \mathrm{C}\left(0^{+}\right) \rightarrow{ }^{12} \mathrm{~B}\left(1^{+}\right)+\nu_{\mu}$ transition. In a $0 \rightarrow 1$ capture, there are three independent observables59: viz. the capture rate, $\Gamma^{\mathrm{cap}}$, the polarization, $P_{\mathrm{av}}$, of the recoil nucleus (here ${ }^{12} \mathrm{~B}$ ) along the muon spin direction, and the longitudinal polarization of the recoil nucleus, $P_{L}$, where

$$
\begin{aligned}
P_{\mathrm{av}} & \equiv\left\langle\mathbf{J} \cdot \boldsymbol{\sigma}_{\mu}\right\rangle / J, \\
P_{L} & \equiv\langle\mathbf{J} \cdot \hat{\mathbf{v}}\rangle / J,
\end{aligned}
$$


and $\left\langle\boldsymbol{\sigma}_{\mu}\right\rangle=\mathbf{P}_{\mu}$ is the muon polarization, $\mathbf{J}$ the nuclear spin, and $\hat{\mathbf{v}}$ the recoil direction. Since the helicity of the muon neutrino, $h_{\nu_{\mu}}$, is $-\frac{1}{2},{ }^{12} \mathrm{~B}$ can have only $m=-1$ or $m=0$ spin directions in the recoil frame. The helicity of the final state $\left({ }^{12} \mathrm{~B}+\nu_{\mu}\right)$ is thus $-\frac{1}{2}$ or $+\frac{1}{2}$, for which the amplitudes are denoted by $M_{-1 / 2}$ and $M_{+1 / 2}$ respectively. The observables are expressed in terms of these amplitudes by

$$
\begin{aligned}
\Gamma^{\text {cap }} & =C\left(2+x^{2}\right) \\
P_{\mathrm{av}} & =P_{\mu} \frac{2}{3}(1+2 x) /\left(2+x^{2}\right) \\
P_{L} & =-2 /\left(2+x^{2}\right)
\end{aligned}
$$

where $x=\sqrt{2} M_{+1 / 2} / M_{-1 / 2}$, and $C$ is a rate constant depending on known quantites. An interesting feature is that $x$ can be determined from the ratio of $P_{\mathrm{av}}$ to $P_{L}$ :

$$
R=P_{\mathrm{av}} / P_{L}=-\frac{1}{3}(1+2 x),
$$

when $P_{\mu}=1$. This ratio is not only more sensitive to $x$ than either observable separately, but it is virtually immune to the systematic uncertainities associated with absolute measurements of recoil polarizations. Roesch et al. 178 determined $x$ in a novel recoil-implantation experiment that enabled $P_{\mathrm{av}}$ and $P_{L}$ to be measured simultaneously. The parameter $x$ depends not only on the pseudoscalar coupling constant, but also on nuclear matrix elements and other weak-interaction form factors, such as weak magnetism. With shell-model calculations of nuclear matrix elements, and with beta-decay experiments to establish the form factors (see sect. 2.3), the dimensionless quantity $m_{\mu} g_{\mathrm{P}} / g_{\mathrm{A}}$ is determined to be $9.0 \pm 1.7$. This result is slightly larger than the PCAC prediction, Eq. 74, but is consistent with the $\mathrm{RMC}$ results in Table 7.

- Another interesting case is the $\mu^{-}+{ }^{16} \mathrm{O}\left(0^{+}\right) \rightarrow{ }^{16} \mathrm{~N}\left(0^{-}\right)+\nu_{\mu}$ transition and its inverse, the beta decay ${ }^{16} \mathrm{~N}\left(0^{-}\right) \rightarrow{ }^{16} \mathrm{O}\left(0^{+}\right)+e^{-}+\bar{\nu}_{e}$, for which both transition rates have been measured179.180. The analysis here depends on the shell-model calculations used to compute matrix elements of parity-changing operators. Gagliardi et al. 180 obtained $m_{\mu} g_{\mathrm{P}} / g_{\mathrm{A}}=11 \pm 2$; however, subsequent evaluations by Warburton et al. 181 and by Haxton and Johnson 182, with much improved shell-model wave functions, resulted in smaller values: in the first case, $m_{\mu} g_{\mathrm{P}} / g_{\mathrm{A}}=7.4 \pm 0.5$ and, in the second, it was in the range $7-9$. Both are consistent with PCAC.

- Lastly, Gorringe et al. 183 recently reported obtaining a value of $m_{\mu} g_{\mathrm{P}} / g_{\mathrm{A}}$ from measurements of the rates and hyperfine dependencies of $\mu^{-}$capture on ${ }^{23} \mathrm{Na}$ to explicit daughter states in ${ }^{23} \mathrm{Ne}$. The $1 S$ atomic ground state of a muonic atom 
in the case of a $J_{i} \neq 0$ nucleus, is split into two hyperfine (HF) states with angular momenta $F_{+}=J_{i}+1 / 2$ and $F_{-}=J_{i}-1 / 2$. For examples where the transition decreases the spin of the nucleus by one unit $\left(e . g \cdot \frac{3}{2}^{+} \rightarrow \frac{1}{2}^{+}\right.$in $\left.\mu^{-23} \mathrm{Na}\right)$, capture from the $F_{-}$state is generally dominated by $g_{\mathrm{A}}$ and the Gamow-Teller (GT) matrix element, whereas capture from the $F_{+}$state is generally dominated by $g_{\mathrm{P}}$ and the same GT matrix element. Consequently, the ratio of $\mu$-capture rates, $\Lambda_{+} / \Lambda_{-}$, is a strong function of $g_{\mathrm{P}} / g_{\mathrm{A}}$ and, to the extent that the GT matrix element dominates other terms, is independent of nuclear wave functions. Hyperfine transitions lead to the depopulation of the upper hyperfine state of the $\mu^{-}$atom $\left(F_{+}\right.$in $\left.{ }^{23} \mathrm{Na}\right)$ in favour of the lower hyperfine state and vield a $\mu^{-}$capture time dependence that is sensitive to $\Lambda_{+} / \Lambda_{-}$. Gorringe et al.183 were able to measure this time dependence in the $\mu^{-}$capture on ${ }^{23} \mathrm{Na}$ to the 1017 $\mathrm{keV} \frac{1}{2}^{+}$and $1833 \mathrm{keV} \frac{3}{2}^{+}$states in ${ }^{23} \mathrm{Ne}$ to obtain $m_{\mu} g_{\mathrm{P}} / g_{\mathrm{A}}=7.6_{-2.5}^{+2.1}$ and $\leq 7.1$, respectively, consistent with the PCAC prediction.

In summary, our knowledge of $g_{\mathrm{P}}$ in nuclei is very sketchy and depends heavily on nuclear-structure evaluations for its determination. There is a hint in light nuclei, ${ }^{12} \mathrm{C}$ and ${ }^{16} \mathrm{O}$, that $g_{\mathrm{P}}$ might be slightly enhanced over the PCAC value, while in heavy nuclei it seems almost completely quenched. Some support for the first remark comes from a calculation by Kolbe, Langanke and Vogel184, who with the continuum random phase approximation are able to reproduce the total capture rates for ordinary muon capture on the light nuclei, ${ }^{12} \mathrm{C},{ }^{16} \mathrm{O}$ and ${ }^{40} \mathrm{Ca}$, without renormalization of $g_{\mathrm{A}}$ or $g_{\mathrm{P}}$. The calculated rates would be significantly lower if in-medium quenching of coupling constants were introduced.

That $g_{\mathrm{P}}$ might be significantly renormalized in heavy nuclei was first discussed by Ericson et al.129, 185, 186, 187, who link the pseudoscalar coupling term to the pion-pole graph, described by Eq.61. In the model of ref.187 the quenching is evaluated to be $g_{\mathrm{P}, \mathrm{eff}}=0.3 g_{\mathrm{P}}$ for infinite nuclear matter. This work is based on only $p$-wave pion-nucleon interactions. Recently, Delorme and Ericson 188 pointed out that the repulsive $s$-wave interaction significantly alters this estimate, and their revised value is now $g_{\mathrm{P}, \text { eff }}=0.6 g_{\mathrm{P}}$. Kirchbach and Riska 189 also stress that the effective induced pseudoscalar coupling constant in nuclei originates from four different sources: the space-like and time-like parts of the axial current and the space-like and time-like parts of the pion pole, each of which is renormalized in a different way in the nuclear medium. The net effect for radiative muon capture in heavy nuclei in their calculation is a substantial quenching of transition rates, which increases with mass number as suggested by the experimental data.

\subsection{Meson-exchange currents in time-like axial-charge transitions}

In contrast to the quenching evident in allowed Gamow-Teller transitions discussed in sect.4.1, transitions originating in the time part of the axial-vector current appear enhanced. Again there are two sources of explanations, both of which contribute 
to the final outcome. First, despite use of the best available shell-model calculations, the shell-model configuration space has inevitably been truncated, and corrections for this truncation are necessary. These are the core-polarization corrections discussed in sect.4.1. Second, the one-body operator from the impulse approximation is augmented by meson-exchange currents in which the axial-vector current prompts a meson to be emitted by one nucleon and reabsorbed on a second nucleon. Such processes lead to two-body operators. The pion, as the lightest-mass meson, produces a long-range operator and so it is the most important meson to consider for exchange currents. In any evaluation of these currents, the pion-absorption amplitude is well known, being determined from the long-range part of the nucleon-nucleon force, but the pion-production amplitude is less certain. In beta decay, the momentum transfer is a few $\mathrm{MeV}$ and is certainly small relative to the nucleon rest mass. If, further, it is assumed that, in describing the pion-production amplitude, it is sufficiently accurate to take the limit that the pion momentum is small as well - i.e. the "soft-pion limit" - then this vertex is determined in a model-independent way through the exploitation of low-energy theorems 190 . The net result is that the pion-production amplitude is given by the commutator, $\left(1 / f_{\pi}\right)\left[Q_{5}, A_{\mu}\right]$, where $A_{\mu}$ is the hadronic axial current, $Q_{5}$ the axial-charge operator and $f_{\pi}$ the pion-decay constant. Were we dealing with zero-mass quarks with point couplings, then the evaluation of this commutator would be trivial: the charges would be generators of closed $S U(2) \times S U(2)$ algebra and the commutator would be determined from the group algebra. These same commutators are nonetheless adopted in this context, following the premise of current algebra191, 192 that the currents given by the quark model exhibit algebraic properties that are of more general validity. From the $S U(2) \times S U(2)$ group algebra, the commutator is

$$
\left[Q_{5}^{a}, A_{\mu}^{b}\right]=i \epsilon_{a b c} V_{\mu}^{c}
$$

where the superscripts are isospin indices and $\epsilon_{a b c}$ the completely antisymmetric tensor in these indices. What this equation expresses is that the leading term in the weak pion-production amplitude is a current proportional to a vector current, $V_{\mu}$.

To continue, we evaluate this commutator between nucleon spinors and take the nonrelativistic limit. For the case in which the Lorentz index, $\mu$, is time-like the commutator is of order $\mathcal{O}(1)$, while when $\mu$ is space-like, it is of order $\mathcal{O}(p / M)$. Here $p$ is the momentum lost by the nucleon making the transition, and is small by comparison with $M$, the nucleon mass. By contrast, an evaluation of the axial current, $A_{\mu}$, itself between nucleon spinors - the impulse approximation - leads, in the nonrelativistic limit, to terms of order $\mathcal{O}(p / M)$ when $\mu$ is time-like, and of order $\mathcal{O}(1)$ when $\mu$ is space-like. Thus, from these limits, it is evident that meson-exchange currents (MEC) will be more important in axial-charge transitions (originating in the time component of the axial current) than in Gamow-Teller transitions (originating in the space component). This observation was first stressed by Kubodera, Delorme and Rho 193.

In sect.4.1, we discussed corrections to some allowed Gamow-Teller matrix elements and from Table 6 we see that the MEC correction is small: of the order of one 
percent. The isobar contribution is not part of the soft-pion MEC correction. This is because the pion-production amplitude at the vertex where a nucleon transforms to an isobar is of order $\mathcal{O}(q / M)$, where $q$ is the pion momentum, and vanishes in the soft-pion limit. However, for the space part of the axial current, the soft-pion terms are suppressed - of order $\mathcal{O}(p / M)$ - and are comparable in size to the correction terms. In this situation the soft-pion theorems by themselves are of little use. Rather, one must start with a model Lagrangian and explicitly evaluate the meson-exchange currents it would generate.

For the rest of this section, we will only consider the time part of the axial current, for which the soft-pion terms are not suppressed: this leads to what are called axial-charge transitions. The two-body operator, after the nonrelativistic limit has been taken, is Fourier transformed to coordinate space and the rank-0 multipole is projected out, giving 194,295

$$
A_{0}(\text { soft }-\pi)=-\frac{1}{2 f_{\pi}} \frac{g_{\pi \mathrm{NN}}}{2 M} \frac{m_{\pi}^{2}}{4 \pi} \boldsymbol{\sigma}_{+} \cdot \hat{\mathbf{r}} Y_{1}\left(x_{\pi}\right) i\left(\boldsymbol{\tau}_{1} \times \boldsymbol{\tau}_{2}\right),
$$

where $\boldsymbol{\sigma}_{+}=\boldsymbol{\sigma}_{1}+\boldsymbol{\sigma}_{2}, \mathbf{r}=\mathbf{r}_{1}-\mathbf{r}_{2}$, and $Y_{1}(x)=(1+1 / x) e^{-x} / x$, with $x=m_{\pi} r$. The corresponding one-body operator from the impulse approximation is

$$
A_{0}(\mathrm{IA})=g_{\mathrm{A}} \frac{\boldsymbol{\sigma} \cdot \boldsymbol{\nabla}}{2 M} \boldsymbol{\tau} .
$$

The interesting question is how big are the matrix elements of the pion-exchange terms relative to those from the impulse approximation. This, then, requires a nuclearstructure calculation for the nucleus in question. Delorme 196 has given an estimate for nuclear matter and finds the ratio $A_{0}(\operatorname{soft}-\pi) / A_{0}(\mathrm{IA})$ to be large, being of order $60 \%$ at nuclear-matter densities. Axial-charge transitions are clearly heavily renormalized by pion-exchange currents. This prediction, which comes from soft-pion theorems and current algebra, is unfortunately hard to test experimentally because such transitions are difficult to isolate. There are two possibilities: one can either examine the small $1 / M$ recoil corrections in an allowed transition, or one can look at transitions in forbidden decays. We will consider examples of both.

\subsubsection{Recoil corrections in allowed transitions}

The $\mathrm{A}=12$ triad has long been the testing ground of nuclear beta-decay theories. It has been used to verify the conserved vector current (CVC) hypothesis through the identification of weak magnetism terms, and it has ruled out any possibility of sizeable second-class currents (see sect.2.3). By yielding the $1 / M$ recoil correction in an allowed Gamow-Teller transition, it has also provided a determination of the axial-

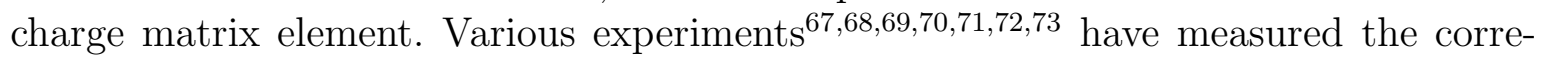
lation between nuclear alignment and the direction of the emitted electron following nuclear beta decay from oriented ${ }^{12} \mathrm{~B}$ and ${ }^{12} \mathrm{~N}$ to the ground state of ${ }^{12} \mathrm{C}$ (see Table 4 ). 
The electron's angular distribution can be represented by a Legendre series, and the coefficient of the $P_{2}(\cos \theta)$ Legendre polynomial is the quantity that must be measured. If we denote this coefficient as $\alpha_{+}$for the positron emission from ${ }^{12} \mathrm{~N}$ and as $\alpha_{-}$for the electron emission from ${ }^{12} \mathrm{~B}$, the difference, $\alpha_{-}-\alpha_{+}$, isolates the weak magnetism matrix element (together with the second-class current term, if present) while the sum, $\alpha_{-}+\alpha_{+}$, isolates the axial-charge matrix element. It is the anti-Hermitian nature of the axial-charge operator that produces this sign change. The average of the experimental alignment data in Table 4 yields $\alpha_{-}+\alpha_{+}=-(2.823 \pm 0.078) \mathrm{GeV}^{-1}$. Theoretically, the combination $\alpha_{-}+\alpha_{+}$is related to nuclear-structure matrix elements via

$$
\begin{aligned}
\alpha_{-}+\alpha_{+} & =-\frac{4}{3} \frac{1}{2 M} y \\
y & =1+2\langle\mathbf{r}(\boldsymbol{\sigma} \cdot \boldsymbol{\nabla})\rangle /\langle\boldsymbol{\sigma}\rangle,
\end{aligned}
$$

where the matrix element $\langle\mathbf{r}(\boldsymbol{\sigma} \cdot \boldsymbol{\nabla})\rangle$ arises from a projection of the rank-1 multipole from the impulse-approximation axial-charge current. The experimental value for $y$ is $y_{\text {expt }}=3.98 \pm 0.11$.

Morita et al.197 calculated these matrix elements in the impulse approxima-

tion with shell-model wavefunctions that describe the ground states as $0 \hbar \omega(0 p)^{-4}$ configurations in the closed-shell at ${ }^{16} \mathrm{O}$. The results span $y=3.6 \pm 0.1$ depending on the choice of residual interaction and are close to the experimental result. There seemingly is little evidence for a significant correction arising from two-body meson-exchange currents. Later, Koshigiri et al.198 realized that the shell-model space was quite restrictive and that enlarging the model space would significantly influence the $\langle\mathbf{r}(\boldsymbol{\sigma} \cdot \boldsymbol{\nabla})\rangle$ matrix element. Using perturbation theory, Koshigiri et al. 198 demonstrated that this correction depended principally on the strength of the residual tensor force. Their final result is $y=3.6-1.2+1.3=3.7$, with -1.2 being their corepolarization estimate with the Hamada-Johnston tensor force and +1.3 being the meson-exchange current enhancement. Guichon and Samour 199 , in a similar calculation, obtained $y=3.0-0.4+1.0=3.6$, where the smaller core-polarization corection of -0.4 arises with the Sussex interaction, which has a weaker tensor component. Clearly, details of the analysis depend on the choice of residual interactions, model spaces, wavefunctions, and so on, but if the predicted meson-exchange enhancement is present, it is in all likelihood masked by the core-polarization correction.

\subsubsection{First-forbidden beta decays}

In the case just covered, the axial-charge operator leads to a small $1 / M$ correction in allowed decay, which has to be isolated from other small $1 / M$ recoil corrections. By contrast, the time part of the axial-vector current contributes a leading term in first-forbidden weak transitions. Unfortunately, the space parts are not negligible: the familiar Gamow-Teller operator, retarded by one unit of orbital angular momentum, 
contributes with comparable magnitude. In first-forbidden decays, the impulse approximation produces six one-body operators: one, the axial-charge operator $\boldsymbol{\sigma} \cdot \boldsymbol{\nabla} / M$, from the time part of the axial-vector current; three from the space part of the axial-vector current, $[\mathbf{r}, \boldsymbol{\sigma}]^{(R)}$, with $R=0,1$, or 2 being the tensorial rank; one from the space part of the weak vector current, $\nabla / M$; and one from the time part, $\mathbf{r}$. Angular momentum selection rules limit the number of these operators that can contribute. For example, in $0^{+}$to $0^{-}$transitions, only two rank-zero operators contribute; in $0^{+}$to $1^{-}$the three rank-one operators contribute; while in $\frac{1}{2}^{+}$to $\frac{1}{2}^{-}$transitions, the five rank-zero and rank-one operators are involved. We limit our discussion to these three cases: thus, the rank-two operator will play no role.

The notation for the first-forbidden beta-decay matrix elements is taken from Warburton et al.200.201 and we define

$$
\begin{aligned}
M_{0}^{T} & =g_{\mathrm{A}}\left\langle J_{f} T_{f}\left\|\frac{1}{M} \boldsymbol{\sigma} \cdot \boldsymbol{\nabla} \boldsymbol{\tau}\right\| J_{i} T_{i}\right\rangle C \\
M_{0}^{S} & =-g_{\mathrm{A}}\left\langle J_{f} T_{f}\|\boldsymbol{\sigma} \cdot \boldsymbol{r} \boldsymbol{\tau}\| J_{i} T_{i}\right\rangle C \\
M_{1}^{u} & =g_{\mathrm{A}} \sqrt{2}\left\langle J_{f} T_{f}\left\|[\boldsymbol{\sigma}, \boldsymbol{r}]^{(1)} \boldsymbol{\tau}\right\| J_{i} T_{i}\right\rangle C \\
M_{1}^{x} & =-\left\langle J_{f} T_{f}\|\boldsymbol{r} \boldsymbol{\tau}\| J_{i} T_{i}\right\rangle C \\
M_{1}^{y} & =-\left\langle J_{f} T_{f}\left\|\frac{1}{M} \boldsymbol{\nabla} \boldsymbol{\tau}\right\| J_{i} T_{i}\right\rangle C,
\end{aligned}
$$

with $C=\left(\hat{J}_{f} /\left(\sqrt{2} \hat{J}_{i}\right)\right)\left\langle T_{i} T_{z i} 1 \mp 1 \mid T_{f} T_{z f}\right\rangle$. The notation is the same as that introduced in Eq.94 for allowed transitions. The conserved vector current hypothesis may be used202 to obtain an alternative expression for $M_{1}^{y}$ in terms of $M_{1}^{x}$ :

$$
M_{1}^{y}=E_{\gamma} M_{1}^{x},
$$

where $E_{\gamma}$ is the photon energy of the analogous $E 1$ gamma transition. In the BehrensBühring 202 formulation, which is being followed here, the beta-decay formulae are derived by expanding the lepton radial wavefunctions in powers of the lepton's mass and energy, and of the nuclear charge. In this treatment, inclusion in the radial integral of an extra factor that depends on the nuclear charge distribution introduces additional matrix elements obtained from $M_{0}^{S}, M_{1}^{x}$ and $M_{1}^{u}$ and denoted $M_{0}^{S \prime}, M_{1}^{x \prime}$ and $M_{1}^{u \prime}$. The ratios of the primed to unprimed quantities, denoted $r_{S}^{\prime}, r_{x}^{\prime}$ and $r_{u}^{\prime}$, are insensitive 200 to details of the nuclear structure and are roughly 0.7 . In the limit that small lepton-energy-dependent terms are dropped - the $\zeta$-approximation of Warburton 201 the formalism for first-forbidden decays exactly matches that for allowed decays: viz. Eqs.93 and 94. Thus,

$$
f t\left(1+\delta_{R}\right)=\frac{6146 \pm 6}{B(0)+B(1)} \mathrm{s}
$$

with $B(0)=|M(0)|^{2}, B(1)=|M(1)|^{2}$, and 


$$
\begin{aligned}
& M(0)=\epsilon_{\mathrm{mec}} M_{0}^{T}+a_{S} M_{0}^{S} \\
& M(1)=a_{u} M_{1}^{u}-a_{x} M_{1}^{x},
\end{aligned}
$$

where

$$
\begin{aligned}
& a_{S}=r_{S}^{\prime} \xi+\frac{1}{3} W_{0} \\
& a_{u}=r_{u}^{\prime} \xi-\frac{1}{3} W_{0} \\
& a_{x}=E_{\gamma}-r_{x}^{\prime} \xi-\frac{1}{3} W_{0} .
\end{aligned}
$$

Here $Z$ is the atomic number of the daughter nucleus in beta decay, $W_{0}$ the end-point electron energy in electron rest-mass units, $\xi=\alpha Z /(2 R)$ with $\alpha$ the fine-structure constant and $R$ the radius of the nuclear charge distribution in electron Comptonwavelength units. The matrix elements given in Eq.109, and the $a$-factors in Eq. 113 are computed in units that render them dimensionless.

Lastly, we have included in Eq.112 a factor, $\epsilon_{\text {mec }}$, representing the expected enhancement of the axial-charge matrix element, $M_{0}^{T}$, over the impulse-approximation value due to meson-exchange currents. The procedure is to compute the matrix elements with the best available shell-model wavefunctions, incorporate core-polarization corrections, and, by comparison of theory with experiment for several beta-decay transitions determine the average value of the enhancement factor. This has been accomplished by Warburton et al. for three mass regions. In the $A=16$ region, the average 203 from three beta decavs and the $\mu^{-}$capture on ${ }^{16} \mathrm{O}$ is $\epsilon_{\mathrm{mec}}=1.61 \pm 0.03$; in the $A=132$ region, the average 204 from three beta transitions is $\epsilon_{\mathrm{mec}}=1.82 \pm 0.07$; and for eighteen transitions in the $A=208$ region, as re-analysed by Warburton and Towner205, the average is $\epsilon_{\text {mec }}=1.79 \pm 0.04$. In the latter two cases, the results are dependent on the choice of the residual interaction used for the core-polarization correction. The values given are based on the Bonn nucleon-nucleon $G$-matrix, which has a weaker tensor component than, for example, the Paris nucleon-nucleon $G$-matrix. For the $A=208$ region, the Paris $G$-matrix leads to a larger enhancement factor of $\epsilon_{\mathrm{mec}}=1.91 \pm 0.04$. These results show that a substantial enhancement of the axial-charge impulse-approximation matrix element is required, and that there is some evidence for larger enhancement factors in heavier nuclei than in light nuclei.

Shell-model calculations of $\epsilon_{\text {mec }}$ have been given by Towner 194 for closed-shellplus-one configurations, for which the evaluation of the matrix element of the soft-pion operator of Eq. 106 involves the calculation of two-body matrix elements between valence and core nucleons and summed over all core nucleons. Such a calculation produces some mass dependence in $\epsilon_{\text {mec }}$ because the number of core orbitals is changing from light to heavy nuclei, and the oscillator frequency parameter used in the radial wavefunction decreases with increasing nuclear size. The results for the soft-pion operator 
are $\epsilon_{\mathrm{mec}}=1.56$ at $A=16$, and $\epsilon_{\mathrm{mec}}=1.76$ at both $A=132$ and $A=208$. This is in reasonable accord with the values deduced from experiment.

Calculations that go beyond soft pions, invoking heavy mesons 194, 206 or chiral perturbation theory 207, and calculations that explain the mass dependence as 'BrownRho' scaling 208 can be examined in the cited references.

\section{Beyond the Standard Model}

\subsection{Right-hand currents}

Maximal parity violation in weak interactions is built into the Standard Model as seems to be demanded by experimental evidence at low energy. At higher energies, one can envisage a restoration of parity symmetry through model extensions. In the charged-current sector, these models introduce additional, predominantly right-handed charged gauge bosons $W_{2}^{ \pm}$to complement the predominantly left-handed gauge bosons $W_{1}^{ \pm}$. The right-handed bosons acquire, via spontaneous symmetry breaking, a mass $m_{2}$ that is larger than the mass $m_{1}(\sim 81 \mathrm{GeV})$ of the observed $W^{ \pm}$. By construction, the left-handed couplings dominate at low energies with parity-symmetry restored at a higher-energy scale. The weak-interaction eigenstates $-W_{L}$, which corresponds to the Standard Model's $W$ gauge boson, and $W_{R}$ - are linear combinations of the physical bosons $W_{1}$ and $W_{2}$ with

$$
\begin{aligned}
& W_{1}=W_{L} \cos \zeta-W_{R} \sin \zeta \\
& W_{2}=W_{L} \sin \zeta+W_{R} \cos \zeta
\end{aligned}
$$

where $\zeta$ is a real mixing angle. The simplest of the model extensions is known as manifest left-right symmetry 209 , in which the physical left-handed and right-handed currents have identical coupling strengths and transformation properties, and one current is obtained from the other via $\gamma_{5} \rightarrow-\gamma_{5}$. In the pure $V-A$ theory, the relevant part of a semi-leptonic weak interaction can be written in a schematic form as

$$
T_{f i}=\frac{g^{2}}{8 m_{1}^{2}}[(V-A)][(V-A)]=\frac{G_{\mathrm{F}}}{\sqrt{2}} V_{u d}[V V+A A-(V A+A V)],
$$

where the $(V-A)$ in the first bracket represents the hadronic current and in the second bracket the leptonic current. The modification to incorporate right-hand currents is

$$
\begin{aligned}
T_{f i}= & \frac{g^{2}}{8 m_{1}^{2}}[c(V-A)-s(V+A)][c(V-A)-s(V+A)] \\
& \quad+\frac{g^{2}}{8 m_{2}^{2}}[s(V-A)+c(V+A)][s(V-A)+c(V+A)] \\
= & \frac{\tilde{G}_{\mathrm{F}}}{\sqrt{2}} V_{u d}\left[V V+\eta_{A A} A A+\eta_{A V}(V A+A V)\right]
\end{aligned}
$$


Table 8. Modifications to various observables of $V-A$ theory through the introduction of right-hand currents evaluated to leading $\operatorname{order}^{a}$ in the small quantities $\zeta$ and $\delta$.

\begin{tabular}{lcc}
\hline & $V-A$ & Modification \\
\hline Fermi decay & $G_{\mathrm{V}}^{2}\left\langle M_{\mathrm{V}}\right\rangle^{2}$ & $G_{\mathrm{V}}^{2}\left\langle M_{\mathrm{V}}\right\rangle^{2}(1+2 \zeta-2 \delta)$ \\
GT decay & $G_{\mathrm{V}}^{2} \lambda^{2}\left\langle M_{\mathrm{A}}\right\rangle^{2}$ & $G_{\mathrm{V}}^{2} \lambda^{2}\left\langle M_{\mathrm{A}}\right\rangle^{2}(1+6 \zeta-2 \delta)$ \\
Muon decay & $G_{\mathrm{F}}^{2}$ & $G_{\mathrm{F}}^{2}(1+4 \zeta-2 \delta)$ \\
Fermi/muon decay & $V_{u d}^{2}\left\langle M_{\mathrm{V}}\right\rangle^{2}$ & $V_{u d}^{2}\left\langle M_{\mathrm{V}}\right\rangle^{2}(1-2 \zeta)$ \\
Neutron decay & $G_{\mathrm{V}}^{2}\left(1+3 \lambda^{2}\right)$ & $G_{\mathrm{V}}^{2}\left[(1+2 \zeta-2 \delta)+3 \lambda^{2}(1+6 \zeta-2 \delta)\right]$ \\
Neutron-decay asym., A & $-2 \frac{\lambda^{2}+\lambda}{1+3 \lambda^{2}}$ & $-2 \frac{\lambda^{2}\left(1+(\zeta+\delta)^{2}\right)+\lambda\left(1+\zeta^{2}+\delta^{2}\right)}{\left(1+(\zeta-\delta)^{2}\right)+3 \lambda^{2}\left(1+(\zeta+\delta)^{2}\right)}$ \\
Fermi long. polariz., $P_{L}^{F}$ & 1 & $1-2(\zeta-\delta)^{2}$ \\
GT long. polariz., $P_{L}^{G T}$ & 1 & $1-2(\zeta+\delta)^{2}$ \\
Ratio $P_{L}^{F} / P_{L}^{G T}$ & 1 & $1+8 \zeta \delta$ \\
\hline
\end{tabular}

${ }^{a}$ Note that radiative corrections are neglected, and $G_{\mathrm{V}}=G_{\mathrm{F}} V_{u d}$.

where $c$ is $\cos \zeta$ and $s$ is $\sin \zeta$, and

$$
\begin{aligned}
\frac{\tilde{G}_{\mathrm{F}}}{\sqrt{2}} & =\frac{g^{2}}{8 m_{1}^{2}}(c-s)^{2}+\frac{g^{2}}{8 m_{2}^{2}}(c+s)^{2} \rightarrow \frac{G_{\mathrm{F}}}{\sqrt{2}}(1+\delta-2 \zeta) \\
\eta_{A A} & =\frac{\epsilon^{2}+\delta}{\epsilon^{2} \delta+1} \rightarrow 1+4 \zeta \\
\eta_{A V} & =-\epsilon \frac{1-\delta}{\epsilon^{2} \delta+1} \rightarrow-1-2 \zeta+2 \delta
\end{aligned}
$$

with $\epsilon=(1+\tan \zeta) /(1-\tan \zeta)$ and $\delta=m_{1}^{2} / m_{2}^{2}$. In Table 8 we show the modifications to various observables in weak interactions evaluated to leading order in the small quantities $\zeta$ and $\delta$. Pure $V-A$ theory is recovered in the limit that $\zeta \rightarrow 0$ and $\delta \rightarrow 0$.

Beta asymmetry. As discussed in sect. 3.2 on neutron decay, the asymmetry in the distribution of electrons relative to a polarization in the parent neutron is governed by a parameter $A$ that depends on the ratio, $\lambda$, of axial-vector and vector coupling constants, $\lambda=G_{\mathrm{A}}^{\prime} / G_{\mathrm{V}}^{\prime}$. With the inclusion of right-hand currents, $A$ becomes a function of $\lambda, \zeta$ and $\delta$ as given in Table 8. Likewise, the ratio of the neutron's $f t$ value to that of the pure Fermi superallowed emitters is also given in terms of $\lambda, \zeta$ and $\delta$. It is then a straightforward matter with these inputs to eliminate $\lambda$ and, allowing for experimental uncertainties, to set limits 210,211,212,213 on $\zeta$ and $\delta$. A similar analysis 214 can be performed for the mirror transition in the decay of ${ }^{19} \mathrm{Ne}$, for which the beta asymmetry and lifetime have been determined. Carnoy et al.14 have also performed a combined fit to both data sets and obtain at the 90\% confidence level: $\delta=0.12 \pm 0.04$ and $\zeta=-0.003 \pm 0.009$. 
The mass-ratio parameter $\delta$ differs from the pure $V-A$ result by nearly three standard deviations and this requires the mass of the predominantly right-hand boson to be in the range 200 to $300 \mathrm{GeV}$.

Longitudinal polarization. Measurements of absolute electron polarizations in Gamow-Teller decays by van Klinken 215 have a combined statistical precision of $0.9 \%$ and absolute accuracy of $1.6 \%$. The most precise measurements are for highly hindered decays for which recoil and forbidden corrections might be important. However, if the evaluation of van Klinken 215 is taken at face value $\left(P_{L}^{G T}=1.001 \pm 0.012\right)$, a limit of $|(\delta+\zeta)| \leq 0.10$ is obtained at the $90 \%$ confidence level.

An entirely different class of experiments is represented by two recent measurements210217 of the ratio of positron polarizations $P_{L}^{F} / P_{L}^{G T}$ for pure Fermi and pure Gamow-Teller transitions. These are believed to be reliable measurements, with precision of less than $0.5 \%$ for the polarization ratio. In addition to being free of absolute analyzing-power uncertainties in the polarimeter, the techniques employed in these measurements reduce many other systematic uncertainties. The results place a limit on the product of the two parameters: $|\zeta \delta|=0.00015 \pm 0.00034$.

$C K M$ Unitarity. If the $C K M$ matrix is taken to be unitary in three generations, then a value for $V_{u d}$ can be obtained from $V_{u d}^{2}=1-V_{u s}^{2}-V_{u b}^{2}$ with recommended values for $V_{u s}$ and $V_{u b}$ taken from ref.6. The result differs from the value determined from superallowed Fermi decays discussed in sect.2.2.4 (see also Fig. 2). If the difference is ascribed to right-hand currents, then a value is obtained for the mixing angle of $\zeta=0.0018 \pm 0.0007$, which is two standard deviations from the pure $V-A$ result.

Muon Spectrum. Without radiative corrections, the muon differential decay rate, $\mu^{+} \rightarrow e^{+}+\nu_{e}+\bar{\nu}_{\mu}$, integrated over $e^{+}$spin directions, is given by

$$
\begin{aligned}
\frac{\mathrm{d}^{2} \Gamma}{x^{2} \mathrm{~d} x \mathrm{~d}(\cos \theta)}= & {\left[(3-2 x)+\left(\frac{4}{3} \rho-1\right)(4 x-3)+12 \frac{m_{e}}{m_{\mu}} \frac{x-1}{x} \eta\right] } \\
& -\left[(2 x-1)+\left(\frac{4}{3} \delta_{m}-1\right)(4 x-3)\right] \xi P_{\mu} \cos \theta .
\end{aligned}
$$

Here, $x$ is the reduced electron energy $E_{e} / E_{\max }$, where $E_{\max }=\left(m_{e}^{2}+m_{\mu}^{2}\right) /\left(2 m_{\mu}\right)=$ $52.83 \mathrm{MeV}$ is the maximum energy, and $m_{e}$ and $m_{\mu}$ are the particle masses. The angle between the positron momentum and the muon polarization vector, $P_{\mu}$, in the $\mu^{+}$rest frame is $\pi-\theta$. The muon-decay Michel parameters are $\rho, \eta, \xi$ and $\delta_{m}$, the values of which in a pure $V-A$ theory are: $\rho=3 / 4, \eta=0, \xi=1$ and $\delta_{m}=3 / 4$. The experiment of Jodidio et al.218 was designed to measure the positron spectrum for $x \geq 0.85$ and $\cos \theta \geq 0.95$, which is sensitive to the combination of parameters, $\xi P_{\mu} \delta_{m} / \rho$. This combination equals 1 for pure $V-A$ and $1-2\left(2 \delta^{2}+2 \delta \zeta+\zeta^{2}\right)$ with the inclusion of right-hand currents. The measured lower limite18 of

$$
\xi P_{\mu} \delta_{m} / \rho \geq 0.99682
$$

sets limits on the right-hand-current parameters at the $90 \%$ confidence level of $\delta \leq 0.04$ or $m_{2} \geq 406 \mathrm{GeV}$ and $-0.056 \leq \zeta \leq 0.040$. 
It should be noted that the limits on right-hand currents from muon decay and $\beta$-asymmetry measurements disagree with each other at the $90 \%$ confidence level. This discrepancy, however, disappears219 at the $3 \sigma$ level.

\subsection{Scalar Interactions}

As has already been noted, the $V-A$ structure of the weak interaction in the Standard Model has been put in a priori to obtain agreement with experiments. Although the model agrees with all experimental results at presently available energies, it remains an interesting quest to search for deviations from the $V-A$ structure. In the last section, we discussed right-hand currents and in this we consider possible scalar and tensor couplings. A more general form of the weak-interaction Hamiltonian was first written down by Jackson, Treiman and Wyld220 as

$$
\begin{aligned}
T_{f i}=\frac{G_{\mathrm{F}}}{\sqrt{2}} V_{u d} & {\left[\bar{\psi}_{p} \gamma_{\mu} \psi_{n} \bar{\psi}_{e^{-}} \gamma_{\mu}\left(C_{V}+C_{V}^{\prime} \gamma_{5}\right) \psi_{\bar{\nu}_{e}}\right.} \\
& +\bar{\psi}_{p} \gamma_{\mu} \gamma_{5} \psi_{n} \bar{\psi}_{e^{-}} \gamma_{\mu} \gamma_{5}\left(C_{A}+C_{A}^{\prime} \gamma_{5}\right) \psi_{\bar{\nu}_{e}} \\
& +\bar{\psi}_{p} \psi_{n} \bar{\psi}_{e^{-}}\left(C_{S}+C_{S}^{\prime} \gamma_{5}\right) \psi_{\bar{\nu}_{e}} \\
& \left.+\frac{1}{2} \bar{\psi}_{p} \sigma_{\mu \nu} \psi_{n} \bar{\psi}_{e^{-}} \sigma_{\mu \nu}\left(C_{T}+C_{T}^{\prime} \gamma_{5}\right) \psi_{\bar{\nu}_{e}}\right] .
\end{aligned}
$$

A pseudoscalar contribution has not been included, since it vanishes in leading order in nuclear beta decay. Further, the induced terms, such as weak magnetism of Eq.10, are not included here. Lastly, time-reversal invariance is assumed so that the coupling constants $C_{i}$ and $C_{i}^{\prime}$ are real. The Standard Model limit is recovered by the replacements: $C_{V}=C_{V}^{\prime}=g_{\mathrm{v}}, C_{A}=C_{A}^{\prime}=g_{\mathrm{A}}$ and $C_{S}=C_{S}^{\prime}=C_{T}=C_{T}^{\prime}=0$. Note that this parameterization also includes right-hand currents when the possibility that $C_{i} \neq C_{i}^{\prime}$ is admitted. In the nonrelativistic limit, $\bar{\psi}_{p} \psi_{n} \rightarrow\left\langle M_{\mathrm{V}}\right\rangle$ and $\bar{\psi}_{p} \sigma_{\mu \nu} \psi_{n} \rightarrow\left\langle M_{\mathrm{A}}\right\rangle$ in nuclear beta decay, so the presence of scalar interactions leads to modifications in Fermi transitions, as tensor interactions lead to modifications in Gamow-Teller transitions.

In 1984, Boothroyd, Markey and Vogel221 set limits on the strength of scalar and tensor interactions in a least-squares adjustment to 93 pieces of data from nuclear beta decay that do not depend on nuclear matrix elements. There are seven parameters to be adjusted if right-hand currents are permitted - namely $C_{i} / C_{V}$ and $C_{i}^{\prime} / C_{V}$ - but just three parameters if $C_{i}^{\prime}=C_{i}$. At the $95 \%$ confidence level $(2 \sigma)$ their results for a seven-parameter fit are

$$
\begin{aligned}
& \left|\frac{C_{S}}{C_{V}}\right| \leq 0.23\left|\frac{C_{S}^{\prime}}{C_{V}}\right| \leq 0.190 \quad\left|\frac{C_{S}+C_{S}^{\prime}}{C_{V}}\right| \leq 0.065 \\
& \left|\frac{C_{T}}{C_{A}}\right| \leq 0.09 \quad\left|\frac{C_{T}^{\prime}}{C_{A}}\right| \leq 0.085 \quad\left|\frac{C_{T}+C_{T}^{\prime}}{C_{A}}\right| \leq 0.01,
\end{aligned}
$$

and for a three-parameter fit 


$$
\left|\frac{C_{S}}{C_{V}}\right| \leq 0.0033 \quad\left|\frac{C_{T}}{C_{A}}\right| \leq 0.0011 .
$$

These limits on scalar and tensor interactions come mainly from measurements on the beta-neutrino angular correlation coefficient, $a$, and the Fierz interference term, $b$. If one writes

$$
K_{i j}=C_{i} C_{j}+C_{i}^{\prime} C_{j}^{\prime}
$$

then these observables are defined in terms of the coupling constants as

$$
\begin{aligned}
a \xi & =\frac{1}{3}\left\langle M_{\mathrm{A}}\right\rangle^{2}\left(K_{T T}-K_{A A}\right)+\left\langle M_{\mathrm{V}}\right\rangle^{2}\left(K_{V V}-K_{S S}\right) \\
b \xi & = \pm 2\left(\left\langle M_{\mathrm{A}}\right\rangle^{2} K_{T A}+\left\langle M_{\mathrm{V}}\right\rangle^{2} K_{S V}\right)
\end{aligned}
$$

with

$$
\xi=\left\langle M_{\mathrm{A}}\right\rangle^{2}\left(K_{T T}+K_{A A}\right)+\left\langle M_{\mathrm{V}}\right\rangle^{2}\left(K_{S S}+K_{V V}\right) .
$$

The upper sign is for electron emission and the lower sign for positron emission. In what follows, we comment on developments since the Boothroyd et al. survey 221 .

$\beta-\nu$ angular correlations. The classic $e-\nu$ correlation data were obtained by observation of the lepton-recoil effect on the energy distribution of stable daughter nuclei from ${ }^{6} \mathrm{He},{ }^{19} \mathrm{Ne},{ }^{23} \mathrm{Ne}$ and ${ }^{35} \mathrm{Ar}$ decays. Such experiments are very difficult because the recoil energies are only a few hundred eV. High precision results are only available for ${ }^{6} \mathrm{He}$ decay 222 and neutron decay 117 . Neither of these are pure Fermi decays and therefore are not sensitive to scalar couplings. However, a recent study of ${ }^{32} \mathrm{Ar}$ $\left(0^{+} \rightarrow 0^{+}\right)$and ${ }^{33} \operatorname{Ar}\left(\frac{1}{2}^{+} \rightarrow \frac{1}{2}^{+}\right)$decays by Schardt and Riisager 24 measured with high precision the shape of the beta-delayed proton peak following the superallowed decay to its isospin analogue state in the daughter nucleus. In this case, the lepton recoil is delivered to a proton-unbound state, and the subsequently emitted proton has a resulting energy spread that exceeds the energy imparted to the recoil daughter by a factor of $\sim 60$. Because its particle decays are isospin-forbidden and the natural width of the proton-unbound level consequently so small, the shape of the proton peak predominantly reflects the $e-\nu-p$ triple-correlation asymmetry parameter, which for isotropic distributions equals the $e-\nu$ correlation coefficient. Adelberger's analysis23 of the Schardt-Riisager data 224 improves the limit on the scalar constant from the seven-parameter fit by a factor of two:

$$
\left|\frac{C_{S}}{C_{V}}\right| \leq 0.12, \quad\left|\frac{C_{S}^{\prime}}{C_{V}}\right| \leq 0.10 .
$$

It is evident that isospin-forbidden $\beta$-delayed proton spectroscopy provides a powerful probe of scalar couplings. 
Fierz interference term. A measurement of the Fierz interference term in a pure Fermi transition would also lead to a limit on scalar couplings. In sect.2.2, a discussion is given on the superallowed Fermi emitters and a statistical rate function, $f$, is defined in Eq. 32. The presence of scalar couplings adds an additional factor to the integrand in Eq. 32 of $(1+\gamma b / W)$, where $\gamma$ is $\left(1-(\alpha Z)^{2}\right)^{1 / 2}$ with $\alpha$ the fine-structure constant and $Z$ the atomic number of the daughter nucleus. Integration over the electron spectrum with the introduction of electromagnetic corrections, as in Eq.37, leads to the modified expression

$$
\left(1-\gamma b\left\langle W^{-1}\right\rangle\right)=\frac{K}{2 G_{\mathrm{v}}^{\prime 2} \mathcal{F} t} .
$$

The presence of scalar coupling in pure Fermi superallowed transitions would therefore show up as a dependence of the $\mathcal{F} t$ values on $\left\langle W^{-1}\right\rangle$ rather than being constant. A fit of the data in Table 2 to $\gamma\left\langle W^{-1}\right\rangle$ gives a result of $b=-0.005 \pm 0.007$ or $|b| \leq 0.017$ at the $90 \%$ confidence level. In the limit of no right-hand currents, $b$ is given by $-2 C_{S} / C_{V}$, with the minus sign for a positron emitter, and hence $\left|C_{S} / C_{V}\right| \leq 0.008$. This result is less restrictive than earlier fits12,225,226.227.228 of this type, mainly because the current set of $\mathcal{F} t$ values shows more dispersion than the earlier data.

Commentary. Any evidence for right-hand currents or scalar interactions mentioned in the last two sections should be taken with extreme caution. Generally, such evidence depends on a number of assumptions, some of which are not always justified. In the past, when an experimental anomaly has been reported it has usually receded in the light of subsequent, improved experiments. As Deutsch and Quin219 put it: "The Standard Model is discouragingly successful".

\section{References}

[1] S. Weinberg, Phys. Rev. Lett. 19, 1264 (1967); A. Salam in Elementary Particle Theory, ed. N. Svartholm (Almquist and Wiksells, Stockholm, 1969) p.367; S.L. Glashow, J. Iliopoulos and L. Maiani, Phys. Rev. D2, 1285 (1970)

[2] B. de Wit and J. Smith, Field Theory in Particle Physics (North Holland, Amsterdam, 1986)

[3] P. Langacker, M. Luo and A.K. Mann, Rev. Mod. Phys. 64, 87 (1992)

[4] M. Kobayashi and T. Maskawa, Prog. Theor. Phys. 49, 652 (1973)

[5] N. Cabibbo, Phys. Rev. Lett. 53, 1802 (1984)

[6] Particle Data Group, Phys. Rev. D50, 1173 (1994)

[7] B.R. Holstein, Weak Interactions in Nuclei (Princeton Univ. Press, Princeton, 1989)

[8] J. Bernstein, Elementary particles and their currents (Freeman, San Francisco, 1968)

[9] T.D. Lee, Particle Physics and Introduction to Field Theory (Harwood Academic, New York, 1981)

[10] S. Weinberg, Phys. Rev. 112, 1375 (1958) 
[11] R.P. Feynman and M. Gell-Mann, Phys. Rev. 109, 193 (1958)

[12] J.C. Hardy, I.S. Towner, V.T. Koslowsky, E. Hagberg and H. Schmeing, Nucl. Phys. A509, 429 (1990)

[13] P.H. Barker and R.E. White, Phys. Rev. C29, 1530 (1984)

[14] P.H. Barker and S.M. Ferguson, Phys. Rev. C38, 1936 (1988)

[15] S.C. Baker, M.J. Brown and P.H. Barker, Phys. Rev. C40, 940 (1989)

[16] P.H. Barker and G.D. Leonard, Phys. Rev. C41, 246 (1990)

[17] G. Azuelos, J.E. Crawford and J.E. Kitching, Phys. Rev. C9, 1213 (1974)

[18] M.A. Kroupa, S.J. Freedman, P.H. Barker and S.M. Ferguson, Nucl. Instr. and Meth., A310, 649 (1991)

[19] Y. Nagai, K. Kunihiro, T. Toriyama, S. Harada, Y. Torii, A. Yoshida, T. Nomura, J. Tanaka and T. Shinozuka, Phys. Rev. C43, R9 (1991)

[20] D.C. Robinson, J.M. Freeman and T.T. Thwaites, Nucl. Phys. A181, 645 (1972)

[21] G. Savard, A. Galindo-Uribarri, E. Hagberg, J.C. Hardy, V.T. Koslowsky, D.C. Radford and I.S. Towner, Phys. Rev. Lett. 74, 1521 (1995)

[22] S.W. Kikstra, Z. Guo, C. van der Leun, P.M. Endt, S. Raman, T.A. Walkiewicz, J.W. Starner, E.T. Jueney and I.S. Towner, Nucl. Phys. A529, 39 (1991)

[23] T.A. Walkiewicz, S. Raman, E.T. Jurney, J.W. Starner and J.E. Lynn, Phys. Rev. C45, 1597 (1992)

[24] V.T. Koslowsky et al., to be published; preliminary results appear in E. Hagberg, V.T. Koslowsky, I.S. Towner, J.G. Hykawy, G. Savard, T. Shinozuka, P.P. Unger and H. Schmeing, Nuclei far from Stability/Atomic Masses and Fundamental Constants (Institute of Physics Conference Series \# 132, ed. R. Neugart and A. Wöhr, 1994) p.783

[25] V.T. Koslowsky, E. Hagberg, J.C. Hardy, H.Schmeing and I.S. Towner, Preprint TASCC-P-94-18, to be published

[26] S.A. Brindhaban and P.H. Barker, Phys. Rev. C49, 2401 (1994)

[27] S. Lin, S.A. Brindhaban and P.H. Barker, Phys. Rev. C49, 3098 (1994)

[28] P.A. Amundsen and P.H. Barker, Phys. Rev. C50, 2466 (1994)

[29] E. Hagberg, V.T. Koslowsky, J.C. Hardy, I.S. Towner, J.G. Hykawy, G. Savard and T. Shinozuka, Phys. Rev. Lett. 73, 396 (1994)

[30] V.T. Koslowsky, J.C. Hardy, E. Hagberg, R.E. Azuma, G.C. Ball, E.T.H. Clifford, W.G. Davies, H. Schmeing, U.J. Schrewe and K.S. Sharma, Nucl. Phys. A472, 419 (1987)

[31] V.T. Koslowsky, R.E. Azuma, H.C. Evans, E. Hagberg, J.C. Hardy, J.R. Leslie, H.Schmeing and C. Virtue, Nucl. Phys. A472, 408 (1987)

[32] H. Schopper, Weak Interactions and Nuclear Beta Decay (North-Holland, Amsterdam, 1966)

[33] I.S. Towner and J.C. Hardy, Nucl. Phys. A205, 33 (1973)

[34] W.E. Ormand and B.A. Brown, Phys. Rev. Lett. 62, 866 (1989); Nucl. Phys. A440, 274 (1985)

[35] I.S. Towner, J.C. Hardy and M. Harvey, Nucl. Phys. A284, 269 (1977)

[36] W.J. Marciano and A. Sirlin, Phys. Rev. Lett. 56, 22 (1986) 
[37] I.S. Towner, Nucl. Phys. A540, 478 (1992)

[38] A. Sirlin, Phys. Rev. Lett. 164, 1767 (1967)

[39] A. Sirlin, Phys. Rev. D35, 3423 (1987); W. Jaus and G. Rasche, Phys. Rev. D35, 3420 (1987); A. Sirlin and R. Zucchini, Phys. Rev. Lett. 57, 1994 (1986)

[40] I.S. Towner, Phys. Lett. B333, 13 (1994)

[41] A. Garcia, R. Huerta and P. Kielanowski, Phys. Rev. D45, 879 (1992)

[42] D.H. Wilkinson, Nucl. Phys. A511, 301 (1990); Nucl. Inst. and Method 335, 172,182,201 (1993); Zeit. Phys. A348, 129 (1994)

[43] W.Y.P. Hwang and H. Primakoff, Phys. Rev. C16, 397 (1977)

[44] H. Behrens and L. Szybisz, Zeit. Phys. A273, 177 (1975)

[45] J. Delorme, Nucl. Phys. B19, 573 (1970)

[46] B.T. Chertok et al., Phys. Rev. C8, 23 (1973)

[47] E. Spamer et al., in Proc of Int Conf of Nuclear Electron Scattering and Photoreaction, Sendai, Japan, eds. K. Shoda and H. Ui (Research Report of Lab. of Nuclear Science, Tohoku Univ., 1972)

[48] U. Deutschmann, G. Lahm, R. Neuhaussen and J.C. Bergstrom, Nucl. Phys. A411, 337 (1983)

[49] L. De Braeckeleer, Phys. Rev. C45, 1935 (1992)

[50] F.P. Calaprice and B.R. Holstein, Nucl. Phys. A273, 301 (1976)

[51] F. Ajzenberg-Selove, Nucl. Phys. A433, 1 (1985)

[52] D.H. Wilkinson, Phys. Lett. B31, 447 (1970)

[53] D.H. Wilkinson and D.E. Alburger, Phys. Rev. Lett. 26, 1127 (1971)

[54] D.H. Wilkinson, Phys. Rev. Lett. 27, 1018 (1971)

[55] I.S. Towner, Nucl. Phys. A216, 589 (1973)

[56] Y.K. Lee, L. Mo and C.S. Wu, Phys. Rev. Lett. 10, 253 (1963)

[57] C.S. Wu, Y.K. Lee and L. Mo, Phys. Rev. Lett. 39, 72 (1977)

[58] W. Kaina, V. Soergel, H. Thies and W. Trost, Phys. Lett. B70, 411 (1977)

[59] L. Grenacs, Ann. Rev. Nucl. Part. Sci. 35, 455 (1985)

[60] T. Mayer-Kuckuk and T.C. Michel, Phys. Rev. 127, 545 (1962)

[61] N.W. Glass and R.W. Peterson, Phys. Rev. 130, 253 (1963)

[62] T. Minamisono et al., in Proc. of Yamada Conf. XXIII Nuclear Weak Process and Nuclear Structure, Osaka, Japan eds. M. Morita, H. Ejiri, H. Ohtsubo and T. Sato (World-Scientific, Singapore, 1989) p.58

[63] T. Minamisono, J. Phys. Soc. Jpn. Suppl. 34, 324 (1973)

[64] R.C. Haskell and L. Madansky, J. Phys. Soc. Jpn. Suppl. 34, 167 (1973)

[65] R.C. Haskell, F.D. Correll and L. Madansky, Phys. Rev. B11, 3268 (1975)

[66] K. Sugimoto, I. Tanihata and J. Goring, Phys. Rev. Lett. 34, 1533 (1975)

[67] P. Lebrun, Ph. Deschepper, L. Grenacs, J. Lehmann, C. Leroy, L. Palffy, A. Possoz and A. Maio, Phys. Rev. Lett. 40, 302 (1978)

[68] H. Brändle, L. Grenacs, J. Lang, L.Ph. Roesch, V.L. Telegdi, P.Truttmann, A.Weiss and A. Zehnder, Phys. Rev. Lett. 40, 306 (1978)

[69] K. Sugimoto, T. Minamisono, Y. Nojiri and Y. Masuda, J. Phys. Soc. Jpn. Suppl. 44, 801 (1978) 
[70] H. Brändle, G. Miklos, L.Ph. Roesch, V.L. Telegdi, P.Truttmann, A. Zehnder, L. Grenacs, P.Lebrun and J. Lehmann, Phys. Rev. Lett. 41, 299 (1978)

[71] Y. Masuda, T. Minamisono, Y. Nojiri and K. Sugimoto, Phys. Rev. Lett. 43, 1083 (1979)

[72] T. Minamisono, J. Phys. Soc. Jpn. Suppl. 55, 382 (1986)

[73] T. Minamisono, A. Kitagawa, K. Matsuta and Y. Nojiri, Hyperfine Interactions 78, 77 (1993)

[74] L. De Braeckeleer, Nucl. Phys. A577, 383 (1994)

[75] T.J. Bowles and G.T. Garvey, Phys. Rev. C18, 1447 (1978)

[76] T.J. Bowles and G.T. Garvey, Phys. Rev. C26, 2336 (1982)

[77] R.E. Tribble and G.T. Garvey, Phys. Rev. C12, 967 (1978)

[78] B.D. McKeown, G.T. Garvey and C.A. Gagliardi, Phys. Rev. C22, 738 (1980)

[79] N. Dupuis-Rolin, J. Deutsch, J.P. Favart and R. Prieels, Phys. Lett. 79B, 359 (1978)

[80] R.E. Tribble and D.P. May, Phys. Rev. C18, 2704 (1978)

[81] R.E. Tribble, D.P. May and D.M. Tanner, Phys. Rev. C23, 2245 (1981)

[82] E.D. Commins and P.H. Bucksbaum, Weak Interactions of Leptons and Quarks (Cambridge University Press, Cambridge, 1983)

[83] P. Renton, Electroweak Interactions (Cambridge University Press, Cambridge, 1990)

[84] M. Gell-Mann and M. Levy, Nuovo Cimento 16, 705 (1960)

[85] B.W. Lee and H.T. Nieh, Phys. Rev. 166, 1507 (1968)

[86] S. Weinberg, Phys. Rev. Lett. 18, 507 (1967)

[87] E. Ivanov and E. Truhlik, Nucl. Phys. A316, 437 (1979)

[88] D.V. Bugg, A.A. Carter and J.R. Carter, Phys. Lett. B44, 278 (1973)

[89] R. Koch and E. Pietarinen, Nucl. Phys. A336, 331 (1980)

[90] V.G.J. Stoks, R.G.E. Timmermans and J.J. de Swart, Phys. Rev. C47, 512 (1993)

[91] R.A. Arndt, R.L. Workman and M. Pavan, Phys. Rev. C49, 2729 (1994)

[92] D.V. Bugg and R. Machleidt, preprint $\pi N N$ Coupling Constants from NN Elastic Data between 210 and $800 \mathrm{MeV}(1994)$

[93] L.A. Ahrens et al., Phys. Lett. B202, 284 (1988)

[94] V. Bernard, N. Kaiser and U-G. Meissner, Phys. Rev. D50, 6899 (1994)

[95] D. Dubbers, Progress in Particle and Nuclear Physics, 26, 173 (1991)

[96] G. Audi and A.H. Wapstra, Nucl. Phys. A565, 1 (1993)

[97] R.S. Van Dyck, D.L. Farnham and P.B. Schwinberg, in Nuclei Far From Stability/ Atomic Masses and Fundamental Constants (Institute of Physics Conference Series \# 132, ed. R. Neugart and A. Wöhr, 1994) p.3

[98] G.L. Greene, E.G. Kessler, R.D. Deslattes and H. Börner, Phys. Rev. Lett. 56, 819 (1986)

[99] K. Schreckenbach and W. Mampe, J. Phys. G18, 1 (1992)

[100] C.J. Christensen, A. Neilsen, A. Bahnsen, W.K. Brown and B.M. Rustad, Phys. Rev. D5, 1628 (1972)

[101] P.E. Spivak, Sov. Phys. JETP 67, 1735 (1988) 
[102] J. Last, M. Arnold, J. Döhner, D. Dubbers and S.J. Freedman, Phys. Rev. Lett. 60, 995 (1988)

[103] R. Kossakowski, P.Grivot, P. Liaud, K. Schreckenbach and G. Azuelos, Nucl. Phys. A503, 473 (1989)

[104] J. Byrne, P.G. Dawber, J.A. Spain, A.P. Williams, M.S. Dewey, D.M. Gilliam, G.L. Greene, G.P. Lamaze, R.D. Scott, J. Pauwels, R. Eykens and A. Lamberty, Phys. Rev. Lett. 65, 289 (1990)

[105] Yu.Yu. Kosvintsev, V.I. Morozov and G.I. Terekhov, JETP Lett. 44, 571 (1986)

[106] V.I. Morozov, Nucl. Inst. and Meth. A284, 108 (1989)

[107] W. Paul, F. Anton, L. Paul, S. Paul and W. Mampe, Z. Phys. C45, 25 (1989)

[108] W. Mampe, P.Ageron, J.C. Bates, J.M. Pendlebury and A. Steyerl, Phys. Rev. Lett. 63, 593 (1989) and Nucl. Inst. and Meth. A284, 111 (1989)

[109] V.V. Nesvizhevskii, A.P. Serebrov, R.R. Tal'daev, A.G. Kharitonov, V.P. Alfimenkov, A.V. Strelkov and V.N. Shvetsov, Sov. Phys. JETP 75, 405 (1992)

[110] W. Mampe, L.N. Bondarenko, V.I. Morozov, Yu. N. Panin and A.I. Fomin, JETP Lett. 57, 82 (1993)

[111] V.E. Krohn and G. R. Ringo, Phys. Lett. 55B, 175 (1975)

[112] D.H. Wilkinson, Nucl. Phys. A377, 474 (1982)

[113] B.G. Erozolimskii, A.I. Frank, Yu. A. Mostovoi, S.S. Arzumanov and L.R. Voitzek. Sov. J. Nucl. Phys. 30, 356 (1979)

[114] P. Bopp, D. Dubbers, L. Hornig, E. Klemt, J. Last, H. Schütze, S.J. Freedman and O. Schärpf, Phys. Rev. Lett. 56, 919 (1986)

[115] B.G. Erozolimskii, I.A. Kuznetsov, I.V. Stepanenko, I.A. Kuida and Yu. A. Mostovoi, Phys. Lett. B263, 33 (1991)

[116] K. Schreckenbach, P.Liaud, R. Kossakowski, H. Nastoll, A. Bussiere and J.P. Guillaud, to be published, 1995

[117] C. Stratowa, R. Dobrozemsky and P. Weinzierl, Phys. Rev. D18, 3970 (1978)

[118] K. Green and D. Thompson, J. Phys. G16, L75 (1990)

[119] X. Song, J. Phys. G13, 1023 (1987)

[120] E.J. Bleser, L.M. Lederman, J.L. Rosen, J.E. Rothberg and E. Zavattini, Phys. Rev. Lett. 8, 288 (1962)

[121] J.E. Rothberg, E.W. Anderson, E.J. Bleser, L.M. Lederman, L.S.Meyer, J.L. Rosen and I.T. Wang, Phys. Rev. 132, 2664 (1963)

[122] A. Alberigi Quaranta, A. Bertin, G. Matone, F.Palmonari, G. Torelli, P.Dalpiaz, A. Placci and E. Zavattini, Phys. Rev. 117, 2118 (1969)

[123] V.M. Bystritskii et al., Sov. Phys. JETP 39, 19 (1974)

[124] G. Bardin et al., Nucl. Phys. A352, 365 (1981)

[125] G. Bardin et al., Phys. Lett. B104, 320 (1981)

[126] D.S. Beder and H. Fearing, Phys. Rev. D35, 2130 (1987)

[127] M.D. Hasinoff et al., Proc. of Third Int. Symp. on Weak and Electromagnetic Interactions in Nuclei, ed. Ts.D. Vylov (World Scientific, Singapore, 1993) p.312

[128] H.J. Lipkin and T.S.H. Lee, Phys. Lett. B183, 22 (1987)

[129] M. Ericson, A. Figureau and C. Thevevet, Phys. Lett. B45, 19 (1973)

[130] M. Rho, Nucl. Phys. A231, 493 (1974) 
[131] K. Ohta and M. Wakamatsu, Nucl. Phys. A234, 445 (1974)

[132] K. Shimizu, M. Ichimura and A. Arima, Nucl. Phys. A226, 282 (1978)

[133] G.F. Bertsch and I. Hamamoto, Phys. Rev. C26, 607 (1982)

[134] D.M. Brink and G.R. Satchler, Angular Momentum (Clarendon, Oxford, 1968)

[135] B.A. Brown, Phys. Rev. Lett. 69, 1034 (1992)

[136] D.H. Wilkinson, Nucl. Phys. A225, 365 (1974)

[137] B.A. Brown and B.H. Wildenthal, At. Data Nucl. Data Tables 33, 347 (1985)

[138] A. Arima, K. Shimizu, W. Bentz and H. Hyuga, Advances in Nuclear Physics 18, 1 (1987)

[139] I.S. Towner and F.C. Khanna, Phys. Rev. Lett. 42, 51 (1979)

[140] I.S. Towner, Phys. Reports 155, 263 (1987)

[141] M. Chemtob and M. Rho, Nucl. Phys. A163, 1 (1971)

[142] F. Osterfeld, Rev. Mod. Phys. 64, 491 (1992)

[143] T.T. Taddeucci et al., Nucl. Phys. A469, 125 (1987)

[144] F. Osterfeld, Phys. Rev. C26, 762 (1982)

[145] F. Osterfeld, D. Cha and J. Speth, Phys. Rev. C31, 375 (1985)

[146] D.E. Bainum et al., Phys. Rev. Lett. 44, 1751 (1980)

[147] C.D. Goodman et al., Phys. Rev. Lett. 44, 1755 (1980)

[148] D.J. Horen et al., Phys. Lett. B95, 27 (1980)

[149] C. Gaarde et al., Nucl. Phys. A369, 258 (1981)

[150] B.D. Anderson et al., Phys. Rev. Lett. 45, 699 (1980)

[151] K.I. Ikeda, S. Fujii and J.I. Fujita, Phys. Lett. 3, 271 (1963)

[152] C. Gaarde, Nucl. Phys. A396, 127c (1983)

[153] A. Bohr and B. Mottelson, Phys. Lett. B100, 10 (1981)

[154] R.D. Smith and J. Wambach, Phys. Rev. C36, 2704 (1987)

[155] M.C. Vetterli et al., Phys. Rev. C40, 559 (1989)

[156] J. Rapaport et al., Nucl. Phys. A410, 371 (1983)

[157] T. Rönnqvist et al., Nucl. Phys. A563, 225 (1993)

[158] C.D. Goodman, J. Rapaport and S.D. Bloom, Phys. Rev. C42, 1150 (1990)

[159] A. García et al., Phys. Rev. Lett. 67, 3654 (1991); E.G. Adelberger, A. García, P.V. Magnus and D.P. Wells, Phys. Rev. Lett. 67, 3658 (1991)

[160] J. Rapaport et al., Phys. Rev. Lett. 47, 1518 (1981)

[161] M.B. Aufderheide, S.D. Bloom, D.A. Resler and C.D. Goodman, Phys. Rev. C46, 2251 (1992); C.D. Goodman, M.B. Aufderheide, S.D. Bloom and D.A. Resler, Phys. Rev. Lett. 69, 2445 (1992)

[162] J. Rapaport and E.R. Sugarbaker, Phys. Rev. Lett. 69, 2444 (1992)

[163] A. García et al., Phys. Rev. C51, 439 (1995)

[164] B.H. Wildenthal, Prog. Part. Nucl. Phys. 11, 5 (1984)

[165] H.P.C. Rood and H.A. Tolhoek, Nucl. Phys. 70, 658 (1965)

[166] H.W. Fearing, Phys. Rev. 146, 723 (1966)

[167] E. Borchi and S. De Gennaro, Phys. Rev. C2, 1012 (1970)

[168] P. Christillin, Nucl. Phys. A362, 391 (1981)

[169] M. Gmitro and P. Truöl, Adv. Nucl. Phys. 18, 241 (1987)

[170] P. Christillin, M. Rosa-Clot and S. Servadio, Nucl. Phys. A345, 331 (1980) 
[171] H.W. Fearing and M.S. Welsh, Phys. Rev. C46, 2077 (1992)

[172] P. Christillin and M. Gmitro, Phys. Lett. B150, 50 (1985)

[173] F. Roig and J. Navarro, Phys. Lett. B236, 393 (1990)

[174] M. Gmitro, A.A. Ovchinnikova and T.V. Tetereva, Nucl. Phys. 453, 685 (1986)

[175] M. Döbeli et al., Phys. Rev. C37, 1633 (1988)

[176] D.S. Armstrong et al., Phys. Rev. C43, 1425 (1991)

[177] D.S. Armstrong et al., Phys. Rev. C46, 1094 (1992)

[178] L.Ph. Roesch, V.L. Telegdi, P. Truttmann, A. Zehnder, L. Grenacs and L. Palffy, Phys. Rev. Lett. 46, 1507 (1981); Helv. Phys. Acta 55, 74 (1982)

[179] L. Palffy et al., Phys. Rev. Lett. 34, 212 (1975)

[180] C.A. Gagliardi, G.T. Garvey, J.R. Wrobel and S.J. Freedman, Phys. Rev. C28, 2423 (1983); H. Heath and G.T. Garvey, Phys. Rev. C31, 2190 (1985); L.A. Hamil,

L. Lessard, H. Jeremie and J. Chauvin, Zeit. Phys. A321, 439 (1985); T. Minamisono, K. Takeyama, T. Ishigai, H. Takeshima, Y. Nojiri and K. Asahi, Phys. Lett. B130, 1 (1983)

[181] E.K. Warburton, I.S. Towner and B.A. Brown, Phys. Rev. C49, 824 (1994)

[182] W.C. Haxton and C. Johnson, Phys. Rev. Lett. 65, 1325 (1990)

[183] T.P. Gorringe et al., Phys. Rev. Lett. 72, 3472 (1994)

[184] E. Kolbe, K. Langanke and P. Vogel, Phys. Rev. C50, 2576 (1994)

[185] M. Ericson, Ann. Phys. 63, 562 (1971)

[186] J. Delorme, M. Ericson, A. Figureau and C. Thevenet, Ann. Phys. 102, 273 (1976)

[187] M. Ericson, Prog. Part. Nucl. Phys. 1, 67 (1978)

[188] J. Delorme and M. Ericson, Phys. Rev. C49,1763 (1994)

[189] M. Kirchbach and D.O. Riska, Nucl. Phys. A578, 511 (1994)

[190] S.L. Adler and Y. Dothan, Phys. Rev. 151, 1267 (1966)

[191] S.L. Adler and R.F. Dashen, Current Algebras (Benjamin, New York, 1968)

[192] V. de Alfaro, S. Fubini, G. Furlan and C. Rosetti, Currents in Hadron Physics (North-Holland, Amsterdam, 1973)

[193] K. Kubodera, J. Delorme and M. Rho, Phys. Rev. Lett. 40, 755 (1978)

[194] I.S. Towner, Nucl. Phys. A542, 631 (1992); Nucl. Phys. A569, 834 (1994)

[195] I.S. Towner, Ann. Rev. Nucl. Part. Sci. 36, 115 (1986); I.S. Towner, Comments Nucl. Part. Phys. 15, 145 (1986)

[196] J. Delorme, Nucl. Phys. A374, 541 (1982)

[197] M. Morita, M. Nishimura, A. Shimizu, H. Ohtsubo and K. Kubodera, Prog. Theor. Phys. Suppl. 60, 1 (1978); M. Morita, M. Nishimura and H. Ohtsubo, Phys. Lett. 73B, 17 (1978)

[198] K. Koshigiri, H. Ohtsubo and M. Morita, Prog. Theor. Phys. 66, 358 (1981); K. Koshigiri, K. Kubodera, H. Ohtsubo and M. Morita, Proc. Int. Conf. on Nuclear Weak Process and Nuclear Structure, eds. M. Morita, H. Ejiri, H. Ohtsubo and T. Sato (World-Scientific, Singapore, 1989) p.52

[199] P.A.M. Guichon and C. Samour, Nucl. Phys. A382, 461 (1982)

[200] E.K. Warburton, J.A. Becker, B.A. Brown and D.J. Millener, Ann. Phys. 187, $471(1988)$ 
[201] E.K. Warburton, Phys. Rev. C44, 233 (1991); Phys. Rev. Lett. 66, 1823 (1991)

[202] H. Behrens and W. Bühring, Electron Radial Wave Functions and Nuclear Beta

Decay (Clarendon, Oxford, 1982)

[203] E.K. Warburton, I.S. Towner and B.A. Brown, Phys. Rev. C49, 824 (1994)

[204] E.K. Warburton and I.S. Towner, Phys. Lett. B294, 1 (1992)

[205] E.K. Warburton and I.S. Towner, Phys. Reports 242, 103 (1994)

[206] M. Kirchbach, D.O. Riska and K. Tsushima, Nucl. Phys. A542, 616 (1992)

[207] T.-S. Park, I.S. Towner and K. Kubodera, Nucl. Phys. A579, 381 (1994)

[208] K. Kubodera and M. Rho, Phys. Rev. Lett. 67, 3479 (1991)

[209] M.A.B. Bég, R.V. Budny, R. Mohapatra and A. Sirlin, Phys. Rev. Lett. 38, 1252 (1977)

[210] B.R. Holstein and S.B. Treiman, Phys. Rev. D16, 2369 (1977)

[211] A.-S. Carnoy, J. Deutsch and B.R. Holstein, Phys. Rev. D38, 1636 (1988)

[212] Yu. V. Gaponov, P.E. Spivak and N.B. Shul'gina, Sov. J. Nucl. Phys. 52, 1042 (1990)

[213] M. Aquino, A. Fernandez and A. Garcia, Phys. Lett. B261, 280 (1991)

[214] A.-S. Carnoy, J. Deutsch, R. Prieels, N. Severijns and P.A. Quin, J. Phys. G: Nucl. Part. Phys. 18, 823 (1992)

[215] J. van Klinken, Nucl. Phys. 75, 145 (1966)

[216] V.A. Wichers et al., Phys. Rev. Lett. 58, 1821 (1987)

[217] A.-S. Carnoy, J. Deutsch, T.A. Girard and R. Prieels, Phys. Rev. Lett. 65, 3249 (1990); Phys. Rev. C43, 2825 (1991)

[218] A. Jodidio et al., Phys. Rev. D34, 1967 (1986); Phys. Rev. D37, 237(E) (1988)

[219] J. Deutsch and P. Quin, in Precision Tests of the Standard Electroweak Model ed. P. Langacker (World-Scientific, Singapore, 1994) to be published

[220] J.D. Jackson, S.B. Treiman and H.W. Wyld, Phys. Rev. 106, 517 (1957)

[221] A.I. Boothroyd, J. Markey and P. Vogel, Phys. Rev. C29, 603 (1984)

[222] C.H. Johnson, F. Pleasanton and T.A. Carlson, Phys. Rev. 132, 1149 (1963)

[223] E.G. Adelberger, Phys. Rev. Lett. 70, 2856 (1993)

[224] D. Schardt and K. Riisager, Z. Phys. A345, 265 (1993)

[225] J.C. Hardy and I.S. Towner, Nucl. Phys. A254, 221 (1975)

[226] L. Szybisz and V. Silbergleit, J. Phys. G7, L201 (1981)

[227] W.E. Ormand, B.A. Brown and B.R. Holstein, Phys. Rev. C40, 2914 (1989)

[228] A.S. Carnoy, J. Deutsch and P. Quin, Nucl. Phys. A568, 265 (1994) 